Portland State University

PDXScholar

\title{
Effects of Daily Social Support on Tension-Reduction Drinking
}

Deborah L. Brannan

Portland State University

Follow this and additional works at: https://pdxscholar.library.pdx.edu/open_access_etds Let us know how access to this document benefits you.

Recommended Citation

Brannan, Deborah L., "Effects of Daily Social Support on Tension-Reduction Drinking" (2011).

Dissertations and Theses. Paper 764.

https://doi.org/10.15760/etd.764

This Dissertation is brought to you for free and open access. It has been accepted for inclusion in Dissertations and Theses by an authorized administrator of PDXScholar. Please contact us if we can make this document more accessible: pdxscholar@pdx.edu. 
Effects of Daily Social Support on Tension-Reduction Drinking

by

Deborah L. Brannan

A dissertation submitted in partial fulfillment of the requirements for the degree of

Doctor of Philosophy

in

Applied Psychology

Dissertation Committee:

Cynthia D. Mohr, Chair

Todd Bodner

Pete Collier

Keith Kaufman

Eric Mankowski

Portland State University

(C) 2010 
Daily Social Support and Drinking, i

\begin{abstract}
Researchers have argued that in times of need having supportive, caring people available can make all the difference between achieving optimum health and well-being or suffering from mental or physical illness (Cobb, 1976; Sarason \& Sarason, 1985; Thoits, 1986). The direct-effect model of support postulates that having the knowledge of available relationship resources (i.e., perceived support) and receiving benefits from those relationships (i.e., received support) both have beneficial effects on health behaviors and well-being (Cohen \& Wills, 1985). According to the stress-buffering model, when stressors are encountered, the certainty of having available resources, as well as having tangible support resources, is hypothesized to lessen the negative effects of stressors (Cohen, et al., 2000; Cohen \&Wills, 1985, Cutrona, 1986; Thoits, 1986).

Most of the research that has examined social support effects on drinkingrelated outcomes has focused on the association between support and alcohol problems, particularly among high risk populations (those who are alcohol dependent, alcohol abusers, or adolescents). Yet, it could be argued that when examining drinking levels, not all consumption is harmful. Of particular concern is drinking that is motivated to reduce negative or stressful experiences. Individuals who use drinking as a method of avoidant coping, or reducing tension drink significantly more alcohol and be at a greater risk for developing later drinking problems (Cooper, Russell, \& George, 1988). Research employing daily process
\end{abstract}


Daily Social Support and Drinking, ii methodology has been able to resolve documented methodological inconsistencies (e.g., Greeley \& Oei, 1999) by examining the within-person processes between negative experiences and alcohol consumption (Tennen \& Affleck, 1996; Tennen, Affleck, Armeli, \& Carney, 2000; Mohr et al., 2008). Similarly, these methodologies have been useful to social support researchers in helping to understand social support as a within-person process rather than just an interpersonal event between two individuals.

This research was part of a larger study about the influence of alcohol use on daily emotion regulation among 47 moderate-to-heavy drinking adults in the local metropolitan area. Participants carried a personal data assistant (handheld interviewer) for 30 days, responding to surveys three times each day (late afternoon, evening). Each survey probed supportive and negative interpersonal exchanges and drinking behaviors. Prior to the daily diary portion of the study, participants completed the Interpersonal Support Evaluation List, a measure of perceived social support. For purposes of analyses, I examined the extent to which exchanges occurring in an earlier interview predicted subsequent solitary drinking at home using data from 2 of the three interviews (predicting evening and late evening drinking only). My analyses revealed that daily socially supportive exchanges had a significant direct effect on subsequent drinking at home alone. Interestingly, the daily supportive exchanges did not buffer the negative exchangeslater drinking relationship. However, my findings revealed that negative exchanges 
Daily Social Support and Drinking, iii also resulted in a reduction in subsequent consumption, which contrasts previous studies that used similar methodologies but with heavier drinkers (e.g., Mohr et al., 2001). Further, perceived support was not related to solitary consumption.

The results of this study indicate that in healthy adults, positively appraised received support directly reduces solitary consumption. This is an important finding given that received support is difficult to document. At the same time, my results showed that among these types of drinkers, negative exchanges may have a stronger direct effect than positive exchanges on solitary consumption.

In non-clinical samples, such as this the relationship between social support and drinking is not straightforward. Thus, using a sophisticated methodology (i.e., daily process methods), this study was able to examine the relationship of drinking and social support on a daily basis; thus, further bridging the gap between social support and the drinking literature. 
Daily Social Support and Drinking, iv

\section{Dedication}

I would like to dedicate this to Mark, Addie, Stepfanie and Aiden. Without your support I could have never made it this far; this has been a team effort. I would also like to thank my brother and his family for being a constant source of support. In addition, I want to thank my grandfather who always pushed me to do more than I ever thought I could. Finally, thank you to my parents who always believed in me. To all those mentioned above, I thank each and every one of you and I offer my most sincere appreciation for your love and support over the years. 
Daily Social Support and Drinking, v

\section{Acknowledgements}

I would like to thank Drs. Cynthia Mohr, Keith Kaufman, Todd Bodner, Eric Mankowski and Pete Collier for their assistance in this project. I would like to specifically thank Dr. Mankowski for consistently being kind and supportive throughout the years. I also thank Dr. Kaufman for always being supportive and helpful, thank you. In addition, I would like to thank Dr. Bodner for always being willing to answer my questions and for being a constant source of valuable information. I cannot thank Dr. Collier enough for being a wonderful addition to my committee. You were always looking out for my best interest. Finally, I would like to thank Dr. Cynthia Mohr. Words cannot express my gratitude. You have been a wonderful mentor and teacher and I will forever be grateful for the time that I have had working with you. You are an inspiration.

This project has been made possible through a larger grant, [Motivational Models of Alcohol Consumption] from National Institute on Alcohol Abuse and Alcoholism (Grant R03-AA014598). I am grateful to Dr. Cynthia Mohr (PI) and her research team who made this project possible.

I would also like Howard Tennen, Mike Todd and Steve Armeli for all your help and support over the years. It is an honor to work with you. I would also like to thank Dr. Dalton Miller-Jones for always being supportive of my endeavors. 


\section{Table of Contents}

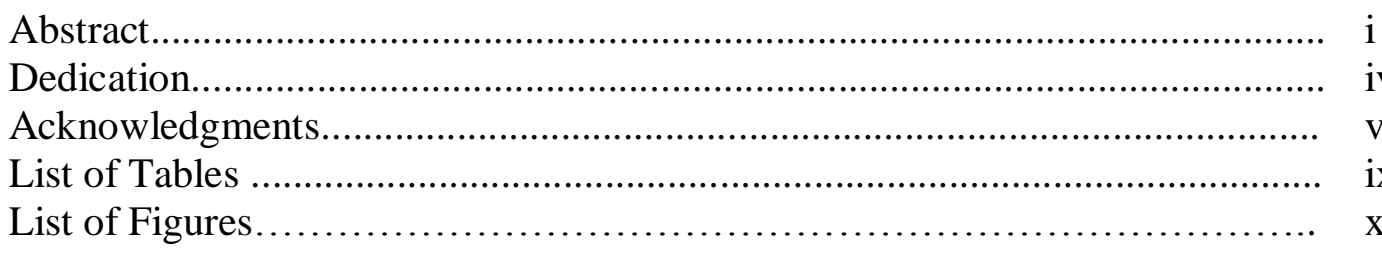

Chapter 1: Introduction............................................................................ 1

Study Overview ................................................ 10

Chapter 2: Social Support................................................................ $\quad 12$

Historical Review..................................................... 13

Construct and Mechanisms of Social Support............................ 16

Construct of Social Support........................................................................ 19

Perceived Social Support...................................................................... 19

Received Social Support...................................................................... 21

Mechanisms of Support.......................................................................... 24

Stress-Buffing Model.............................................................................. 25

Direct Effect Model....................................................................... 26

Summary of Support Mechanisms.................................... 28

Social Support and Health Behaviors.......................................................... 29

Chapter 3: Alcohol Consumption.............................................................. $\quad 32$

Drinking Motives...................................................................................... 33

Tension-Reduction Drinking ............................................................... $\quad 35$

Chapter 4: Daily Process Methodology........................................................... 41

Daily Process Methodology and Social Support.......................................... 43

Daily Process Methodology and Alcohol Consumption................................ 46

Chapter 5: Purpose and Hypotheses............................................................ 50

Hypotheses........................................................ 51

Received Social Support.......................................... 51

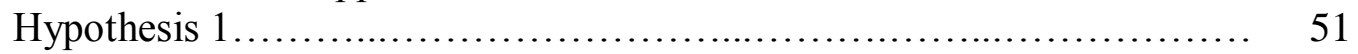

Stress-Buffering Model........................................... 52

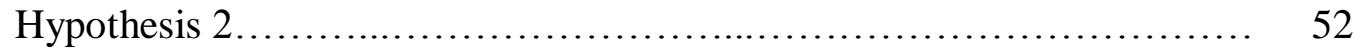

Perceived Social Support......................................... 53

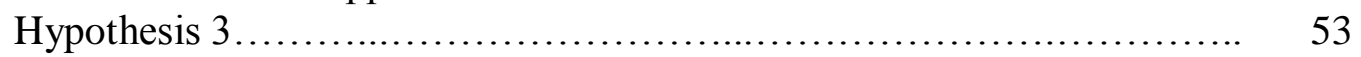

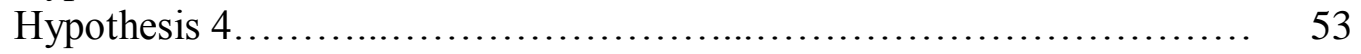

Chapter 6: Methods................................................... 54 


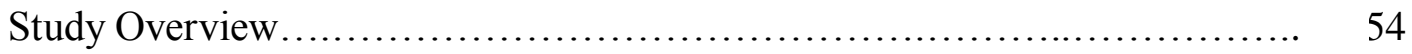

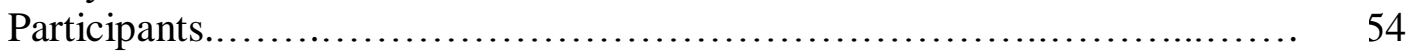

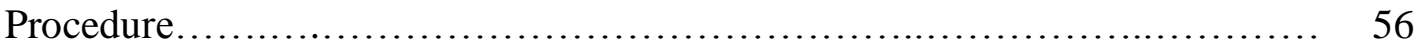

Initial Assessment......................................................... 57

Daily Interview........................................................ 58

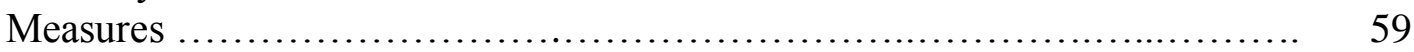

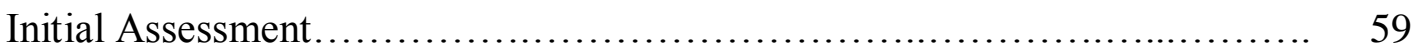

Perceived Social Support................................................ 59

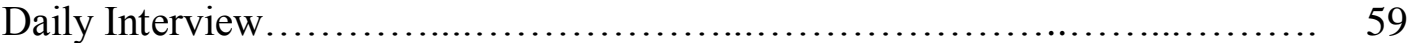

Socially Supportive Exchanges.......................................... 59

Negative Interpersonal Exchanges...................................... 60

Daily Alcohol Consumption......................................... 61

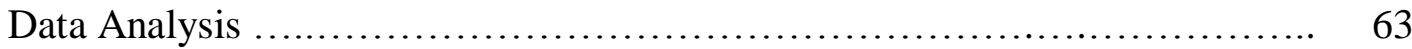

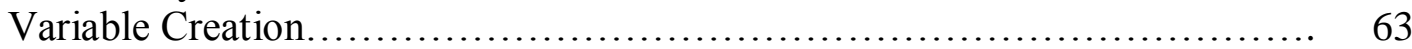

Drinking Variable ................................................... 63

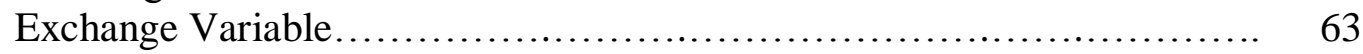

Between-Person Variable............................................... 64

Lagged Variables........................................................ 64

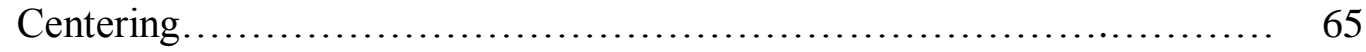

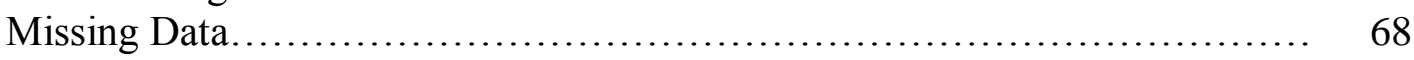

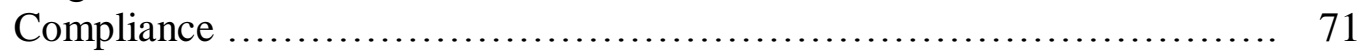

Answering Questions on the Surveyor.............................. 72

Missing Data for Each Variable.......................................... 73

Survey Completion Rates............................................... 74

Distribution of Measure and Test of Outliers................................ 76

Data Structure........................................................... 84

Trends and Serial Dependency............................................. 85

Day of Week Contrasts.............................................. 86

Trends over the course of the study ................................. 87

Time-of-Day Covariates............................................. 88

Autoregressive Error Analyses....................................... 89

Chapter 7: Results............................................................ 91

Descriptive Information................................................ 91

Possible Covariates................................................... 92

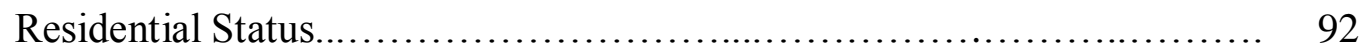

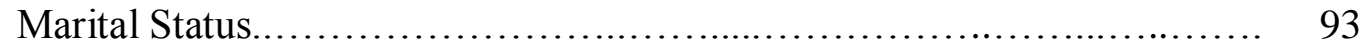

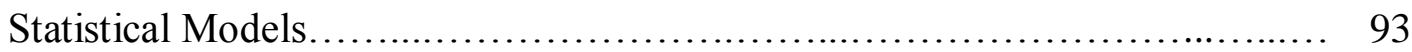

Hypothesis Tests....................................................... 94

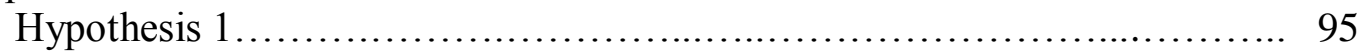

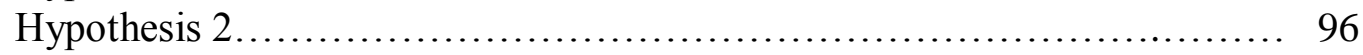

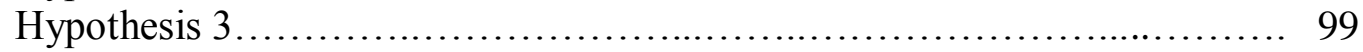


Daily Social Support and Drinking, viii

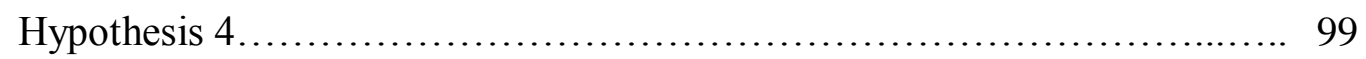

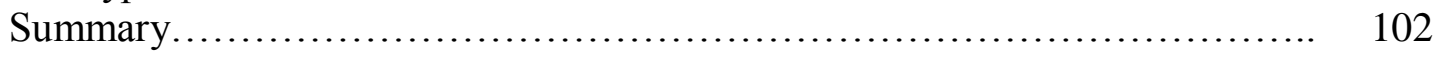

Chapter 8: Discussion ................................................. 105

Direct-Effect Model.................................................. 107

Stress-Buffering Model.............................................. 111

Perceived Social Support.............................................. 119

Implications of Dissertation............................................ 122

Limitations............................................................. 125

Strengths and Future Research......................................... 129

References...................................................... 151

Appendix A: Sample recruitment advertisement............................. 185

Appendix B: Handheld interviewer, interpersonal exchange questions.......... 186 
Daily Social Support and Drinking, ix

\section{List of Tables}

Table 1. Between-Person Survey Completion Rates..................... 133

Table 2. Within-Person Survey Completion Rates...................... 134

Table 3: Frequencies and Percentages for Interpersonal Exchanges.......... 135

Table 4. Correlation table.......................................... 136

Table 5. Hypothesis 1, Direct Effect Model.............................. 137

Table 6a. Hypothesis 2, Stress-Buffering Model........................ 138

Table 6b: Hypothesis 2, Continued ............................... 139

Table 7. Hypothesis 3, Perceived Social Support...................... 140

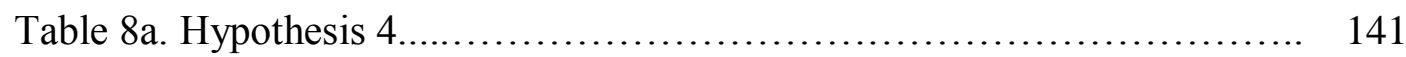

Table 8b. Hypothesis 4, Continued................................. 142

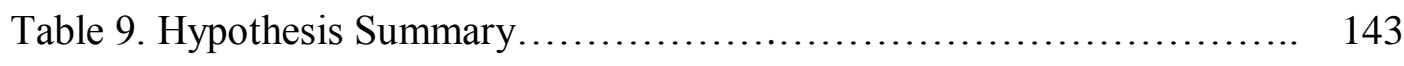


Daily Social Support and Drinking, $\mathrm{x}$

\section{List of Figures}

Figure 1. Conceptual model of where social support and drinking may influence the

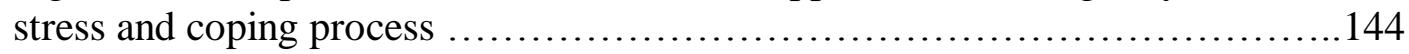

Figure 2. Two constructs of social support (perceived and received) can be examined within the direct effect and stress-buffering models................... 145

Figure 3. Four Factor Model of Alcohol Consumption (Cooper, 1994) ........ 146

Figure 4. Daily Survey Schedul......................................... 147

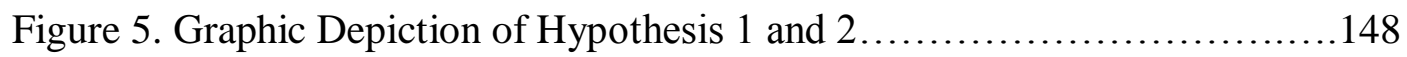

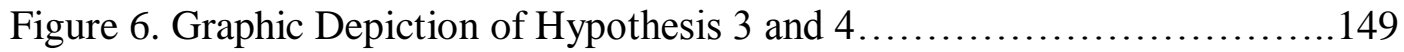

Figure 7. Hypothesis 4, Step 1: Simple slopes for Perceived Social Support and

Negative Interpersonal Exchanges.................................... 150 
Daily Social Support and Drinking, 1

\section{CHAPTER 1}

Introduction

Social ties in the form of close personal relationships play a significant role in a person's life; these relationships offer security, safety, and social support while contributing to our well-being (e.g., Cohen \& Hoberman, 1983; Rook \& Pietromonaco, 1987; Sarason, Sarason, \& Gurung, 2001). These important relationships influence emotions and adjustment, help with stress and offer information about coping (Cohen \& Wills, 1985; Lakey \& Cohen, 2000). Baumeister and Leary (1995) suggest that close personal ties actually fulfill a fundamental human need; it is through these relationships people that find meaning in their lives (Brissette, Cohen \& Seeman, 2000; Rook \& Pietromonaco, 1987; Ryff, 1989). In contrast, research indicates that a lack of close relationships can lead to many negative outcomes such as mortality, maladaptive coping behaviors (e.g., alcohol abuse, dependence), depression and suicide (Baumeister \& Leary, 1995; DeLongis, Folkman, \& Lazarus, 1988; Sarason et al., 2001).

Researchers have argued that in times of need having supportive, caring people available can make all the difference between achieving optimum health and well-being or suffering from mental or physical illness (Cobb, 1976; Sarason, et al., 1990; Thoits, 1986). Consistent with this notion, in 1976, Dr. Sidney Cobb argued that the social support provided by others during stressful times can actually protect 
Daily Social Support and Drinking, 2

(i.e., buffer) individuals from negative consequences. Cassell and Cobb established the foundation for future research in the area of social support (Cassell, 1974, 1976; Cobb, 1976). They identified the broad model of social support which led to the development of a multifaceted construct of social support that consisted of perceived support (i.e., appraisals), received support (behaviors) and the social support network (i.e., resources that are made available by those with whom you have a relationship; Cohen \& Wills, 1985; Pierce, Sarason, Sarason, Joseph, \& Henderson, 1996; Vaux, 1988).

Furthermore, social support processes were further clarified through the lens of the transactional model of stress and coping (Lazarus \& Folkman, 1988). One consequence was that many studies transitioned from conceptualizing support as a static personality characteristic to a dynamic relationship between the person and the environment (Vaux, 1988). Accordingly, social support is not a personality trait, nor an environmental state; rather it is a transactional process between the person and their environment (Lazarus \& Folkman, 1987; Vaux, 1988). Support appraisals were considered subjective descriptions of how individuals assess situations. According to the transactional model of stress and coping, in any given situation we must take into account both the person and the environmental influences. The person influences the environment and the environment influences the person. For instance, Lazarus and Folkman (1987) elucidated that a "threat" is a union of both the person and the environment. In other words, it takes specific contextual (i.e., environmental) 
Daily Social Support and Drinking, 3

qualities as well as a specific characteristics of an individual who will appraise the situation as a threat for a stressor to have a negative influence on mood and wellbeing. It is possible for one individual to appraise a problem as a threat, yet another person who is exposed to the same conditions to appraise the situation as challenging or benign.

Thus, one key component of the stress and coping process, which includes social support, is cognitive appraisal (See Figure 1). Appraisals are the cognitive processes by which an individual acquires information that may affect his or her well-being. Social support can positively affect appraisals and enhance coping performance (Cohen, Gottlieb, \& Underwood, 2000). More specifically, Lazarus and Folkman differentiate between two types of appraisals: primary and secondary. Primary appraisals are the assessments that determine the importance of the situation (i.e., whether well-being is jeopardized). Primary appraisals are concerned with the relative impact of stressors, specifically whether stressors are benign, positive, threatening, harmful, or challenging to well-being. Secondary appraisals are another type of appraisal whereby an individual decides whether the details of a situation or its emotional consequences can be managed. Accordingly, social support is a transaction between a person and his or her beliefs about resources or the degree to which actual support helps in times of need, in combination with his or her environment. 
Daily Social Support and Drinking, 4

This model facilitated a more process-oriented perspective on social support (Cohen \& Wills, 1985; House Umberson, \& Landis, 1981). In an extensive review of previous studies, Cohen and Wills (1985) posited that there was a relationship between social support and well-being and health behaviors. More specifically, they argued that there are two distinct mechanisms by which social support functions (i.e., direct-effect and stress-buffering models), and it is through these models that health and well-being can be understood. Moreover, they found that both models are similar, yet are functionally different. More specifically, the direct-effect model of support postulates that having relationships has a beneficial effect on health behaviors and well-being. The hypothesized mechanism specifies that being socially integrated allows an individual to have higher levels of perceived and received support, which results in better health behaviors (Cohen \& Wills, 1985; Rodriguez \& Cohen, 1998). In analytic terms, the focus of this model is on the main effect of relationships on health and well-being. In their review, Cohen and Wills (1985) found a number of studies documenting empirical evidence consistent with this notion (see Bell, LeRoy \& Stephenson, 1982; Miller \& Ingham, 1976).

In contrast, the stress-buffering model of social support suggests that support is beneficial for physical and mental health because it shields individuals from the damaging effects of stress (Cohen \& Wills, 1985; Rodriguez \& Cohen, 1998). Whether the support is actually given or an individual simply believes that it will be there in times of need, stress may be buffered in both the short term and the long 
Daily Social Support and Drinking, 5

term. In addition, Cohen and Wills (1985) concluded that the buffering model, just as the direct effect model, can take the form of perceived or received support as depicted in Figure 2.

Over time there has been a remarkable amount of research focused on the possible positive effects of support on health and well-being (e.g., Broadhead, Kaplan, James, Wagner, Schoenbach, Grimson, Heyden, Tibblin, \& Gehlback, 1983; Cohen, et al., 2000; Cohen \& Syme, 1985); as well as health-compromising behaviors such as smoking and drinking (Chi, Kaskutas, Sterling, Campbell \& Weisner, 2008; Groh, Jason, Davis, Olson, \& Ferrari, 2007; Handey \& Chassin, 2008; Mulia, Schmidt, Bond, Jacobs \& Korcha, 2007; Pierce, et al., 1996; Wills, Resko, Ainette \& Mendoza, 2004; Windle \& Windle, 1996). Most of the research that has examined social support effects on drinking-related outcomes has focused on the association between support and alcohol problems, particularly among high risk populations (those who are alcohol dependent, alcohol abusers, or adolescents). Yet, it could be argued that when examining drinking levels, not all consumption is inherently harmful. There is a significant amount of research indicating that there are many health benefits associated with moderate consumption (see Camargo, Hennekens, Gaziano, Glynn, Manson, \& Stampfer, 1997; Ikehara, Iso, Yamagishi, Yamamoto, Inoue, \& Tsugane, 2009; Yuan, Ross, \& Gao, 1997).

Furthermore, researchers have focused on the biological and physiological benefits of moderate drinking--not much effort has gone in to examining the 
Daily Social Support and Drinking, 6 psychosocial costs and benefits of moderate drinking in normative samples. Pierce et al., (1996) pointed out that much of the research that has examined psychological measures such as stress and drinking has used samples that were so unique (i.e., arthritic patients, depressed patients, alcoholic patients) that generalizing to a normative sample was not possible. Moreover, Pierce et al. (1996) argued that future research should examine different types of drinking behaviors and motives.

Much of the previous research that has linked social support to alcohol consumption has examined very specific types of drinking behavior. Interestingly, most researchers have been concerned with problem populations and their specific drinking behaviors (i.e., adolescent drinking; college student binge drinking). To more fully understand the relationship between alcohol behaviors and social support, it could be argued that researchers must disentangle discrete drinking behaviors. Cooper and colleagues have argued that "drinking behaviors" should not be considered one distinct behavior but many psychologically unique behaviors (Cooper, Frone, Russell, \& Mudar, 1995).

In early research that examined specific drinking motivations, Cox and Klinger $(1988,1990)$ conjectured that these motives can be distinguished by two dimensions: the valence (positive or negative) and source (internal or external) of the outcomes (Cooper, 1994). Cooper (1994) then built on the foundation put forth by Cox and Klinger (1988), by creating and testing a 4-factor model of drinking motives (see Figure 3). Specifically, Cooper (1994) probed possible internal and external 
Daily Social Support and Drinking, 7

sources of drinking motives in combination with positive and negative valence.

Cooper (1994; Cooper et al., 1995) put forth that behaviors that were indicative of drinking to enhance positive moods are internally and positively reinforced motivation. An example of this specific type of behavior would be if a person received a promotion at work and they drink to celebrate the occasion. Drinking motives because of social pressure (i.e., conformity), which is considered external and negative reinforcement, is also included in this model. Moreover, the 4-factor model also includes an external and positive reinforcement motivation; this represents drinking in order to enhance social experiences. Lastly, Cooper established that drinking as a method of coping was a significant and distinct motivation which was associated with internal and negative reinforcement. The research suggests that it was the drinking-to-cope motives that was the most linked to problematic drinking behaviors.

Moreover, drinking behaviors are viewed as part of a maladaptive coping strategy that some turn to in lieu of more adaptive, problem-focused strategies. According to the tension-reduction hypothesis (see Chapter 3) individuals drink to alleviate the negative effects of stressful experiences (Greeley \& Oei, 1999). Research suggests that individuals who use drinking as a method of avoidance coping or reducing tension are more likely to drink significantly more alcohol and be at a greater risk for developing later drinking problems than those who do not use alcohol as a method of coping (Cooper, Russell, \& George, 1988). Moreover, those 
Daily Social Support and Drinking, 8 individuals who drink to cope or reduce psychological tension are also more likely to drink alone (e.g., Mohr et al., 2001). Research conducted by Mohr et al. (2001) demonstrated the link between tension-reduction drinking and solitary consumption; more specifically, they also found a link between negative interpersonal exchanges and drinking in a solitary context. Since it was their goal to examine daily stressors, negative interpersonal exchanges were used because according to the literature, negative interpersonal interactions are some of the most damaging, long lasting of all stressors (see DeLongis, Coyne, Dakof, Folkman, \& Lazarus, 1982). Moreover, in a study by DeLongis and colleagues, they found that daily stressors were linked to illness and overall health (DeLongis, et al., 1982). Thus, I operationalized daily stressors by examining negative interpersonal exchanges.

It is important to note that that throughout this dissertation I discussed different types of drinking and different kinds of drinking. These words were used interchangeably because it was not the goal of this dissertation to attempt to create a new typology of drinking behaviors but rather examine, from a social psychology perspective, possible predictors and buffers of solitary drinking at home behaviors.

The advent of daily process methods has been key to both the social support and the drinking-to-cope literatures. Although most research on social support and tension-reduction drinking has been conducted using traditional cross-sectional and less frequently, multi-wave longitudinal methods of data collection, researchers are increasingly examining the within-person processes in alcohol consumption (Armeli, 
Daily Social Support and Drinking, 9

Todd, \& Mohr, 2005; Mohr et al., 2001; Park, et al., 2004) and social support (Coyne \& DeLongis, 1986; Newsom, Nishishiba, Morgan, \& Rook, 2003). Moreover, since these behaviors are considered processes, they need to be measured accordingly (Tennen et al., 2000). In other words, coping-related behaviors should be measured as close to the time of the occurrence so as to capture the within-person processes (Stone, Schwartz, Schwartz, Schkade, Kahneman \& Krueger, 2006; Tennen, Affleck, Coyne, Larsen, \& DeLongis, 2006). Support and drinking are linked the stress and coping process (see Figure 1) and they fluctuation throughout the day; thus, the best way to assess these processes is to capture information as close to the experience as possible rather than retrospectively (e.g., Tennen et al., 2000; Bolger, Davis, \& Rafaeli, 2003).

In many of the studies examining tension-reduction drinking and coping there are still other significant issues such as the lack of ecological validity (Mohr, Armeli, Tennen, \& Todd, 2009; Greeley \& Oei, 1999; West \& Sutker, 1990), as well as design problems. Some argue that traditional designs (i.e., cross-sectional, longitudinal designs) do not capture the fluctuating drinking and coping processes (Lazarus, Lazarus, Campus, Tennen \& Tennen, 2006; Mohr et al., 2009; Ptacek, Smith, Espe, \& Raffety, 1994; Tennen et al., 2000; Todd, Armeli, Tennen, Carney \& Affleck, 2003). Daily process methodology provides one way to address the aforementioned issues. This methodology affords considerable insight into experiences and behaviors that happen each day. Moreover, taking multiple 
Daily Social Support and Drinking, 10

measures per day allows one to estimate the temporal relationships among stressors (i.e. negative interpersonal exchanges) and social support, which are likely vital components in understanding alcohol-related behaviors. In other words, I was able examine the effects of social support on subsequent drinking. This dissertation addressed these issues because the data were collected using daily process methodology; thus, it was possible to consider within-person relationships among negative interpersonal exchange, social support and drinking behaviors.

\section{Study Overview}

This research was part of a larger study about the influence of alcohol use on daily emotion regulation, directed by Cynthia D. Mohr, Ph.D. (NIAAA Grant R03AA014598). I was integrally involved in all phases of this research project. This project examined daily interactions, moods, coping strategies and health behaviors of adults living in the surrounding communities. The 49 eligible individuals agreed to carry a personal data assistant (handheld interviewer) for 30 days; three times each day (late afternoon, evening) the handheld interviewer would alert the participant to an imminent survey. Each survey probed interpersonal exchanges and drinking behaviors. Using this method, I was not only be able to examine how daily socially supportive exchanges relate to tension-reduction drinking behaviors but I was able to examine how daily interpersonal exchanges affect later solitary drinking at home (i.e., tension-reduction drinking). 
Daily Social Support and Drinking, 11

In this study, daily diary data are hierarchically structured, with 90 interpersonal exchanges and drinking behaviors nested within each person; thus, data contain both within- and between-person levels. An additional complication with a data set such as this is that within person data are often unbalanced in nature, meaning that there are missing observations. I proposed to use Hierarchical Linear Modeling (HLM, v6.0; Raudenbush, Bryk, Cheong, \& Congdon, 2000), which has been developed to handle unbalanced data of this nature.

In sum, this dissertation examined extent to which social support had a direct and buffering effect on drinking. I attempted to make a significant contribution to the field by using daily process methodology to further probe this relationship. 
Daily Social Support and Drinking, 12

\section{CHAPTER 2}

\section{Social Support}

Whether it is across cultures (Markus \& Kitayma, 1991; Triandis, 1989) or over time (Reis, Sheldon, Gable, Roscoe, \& Ryan, 2000) close personal ties are viewed as a fundamental component in what makes a person happy and healthy and what gives a person's life meaning (Brissette et al, 2000; Rook \& Pietromonaco, 1987; Ryff, 1989). On the other hand, not having close relationships can pose a significant threat for a multitude of problems such as illness, maladaptive behaviors, unhealthy habits and suicide (Baumeister \& Leary, 1995; DeLongis et al, 1988; Sarason et al., 2001). Due to the vast benefits people can offer to one another, relationships offer a significant potential for intervention to improve wellness and decrease or thwart negative, self-harming behaviors.

Multiple theoretical models argue the importance of close relationships; for example, Baumeister and Leary (1995) posit that close relationships fill a basic human need. In fact, when people were asked they have consistently reported that there is almost nothing more important in their lives as their close personal relationships and they need these relationships to be truly fulfilled (Brissette, et al., 2000; Rook \& Pietromonaco, 1987; Ryff, 1989). Similarly, Self Determination Theory asserts that all people have three basic psychological needs: autonomy, competence, and relatedness. Relatedness, otherwise known as "a need to belong;" is a universal need to form sturdy and constant interpersonal relationships (Deci \& 
Daily Social Support and Drinking, 13

Ryan, 2000; LaGuardia \& Patrick, 2008). Well-being research also echoes this sentiment; Ryff (1988) and others have claimed that individuals are able to achieve optimal psychological well-being and thrive when they have strong, positive relationships with others (Erikson, 1959; Diener, 1984; Ryff \& Heidrich, 1997; Ryff, 1989).

In the following section I will offer a historical account of how early researchers understood that supportive relationships are key to health and well-being. Then I offer an explanation of the overarching construct of social support, and the mechanisms by which social support operates.

\section{Historical Review}

Over 100 years ago, Emile Durkheim began examining close personal relationships (Durkheim, 1951; Uchino \& Kazdin, 2004; Vaux, 1988). Durkheim (1951) contended that social connectedness was so important that without it people would be driven to suicide. This view resonated with many but did not seem to receive much attention from researchers or the health community until decades later. In 1976 two seminal papers were published by Drs. John Cassell and Sidney Cobb that endorsed the importance of social connectedness and support (Sarason, Sarason, \& Pierce, 1990). More specifically, Dr. Cassell $(1974,1976)$ argued that social groups serve a protective function (e.g., buffer against stressors and life events), thus enabling a person to reach optimal health and well-being. Furthermore, a disturbance or termination of these social ties could result in an increased vulnerability to illness 
Daily Social Support and Drinking, 14

and disease. In particular, in his 1976 American Psychosomatic Society presidential address, Dr. Cobb put forth that it is the information that a support network provides in stressful times that can buffer an individual against the potential negative consequences. He went on to say that the belief that one is part of a large social network of supportive individuals who love and care about the person can enhance self-worth and can reduce the impact of stress, as well as help a person throughout the coping process.

Cassell and Cobb established the groundwork for future research in the area of social support (Cassell, 1974, 1976; Cobb, 1976). They defined the broad concepts of social support and called for others to take interest in this important area of research. It was because of their preliminary work, in combination with Durkheim's initial work in social connectedness, social support has been (and remains) a dominating area of interest in the social sciences (Vaux, 1988). Unsurprisingly, early social support research was plagued by conceptual inconsistencies and ambiguity (Cutrona, 1986; Eaton, 1978; Sarason et al., 1990; Vaux 1988; Wellman \& Wortley, 1990). More specifically, there were inconsistencies in how social support was defined (Thoits, 1982; Vaux, 1988) and how the construct of social support was operationalized (Dunkel-Schetter, Sagrestano \& Feldman, 1996; Wills \& Shinar, 2000). For example, some viewed social support through the lens of social exchange theory that was brought forth by Thibaut and Kelley (1959). Their seminal work in this area suggested that anticipated 
Daily Social Support and Drinking, 15

reciprocity, perception of efficacy, and direct rewards were a few of the reasons why individuals engage in relationships. Social support researchers built on that idea and proposed that social support involves multifaceted, long-term transactions between individuals that require giving and taking by each person involved; a shared obligation is necessary by each party and they must be willing and able to receive and offer support as needed (Thibaut \& Kelley, 1959; Vaux, 1988). While this case was being made, Shumaker and Brownell (1984) suggested that social exchange theory was a limited view of social support. It boiled down a complex process to a simple cost/benefit analyses (Vaux, 1988). This is an example of the conceptual and theoretical inconsistencies in the social support literature. Consequently, researchers insisted that there needed to be a more unified, clear definition of the support construct and theory. Sarason et al. (1990) suggested that social support is "like a root-bound potted plant, [and it] can profit not only from being divided but also from the fertilization of its theoretical underpinnings" (p. 97). Vaux (1988) argued that "if social support has failed to meet our expectations, it is because those expectations were too high and our views too simplistic" (p. 31).

Understanding these issues and wanting some resolution, Thoits (1982) offered that social support is “the degree to which a person's basic social needs are gratified through interaction with others" (p.147). This definition seemed to take root and develop and many began to elaborate on her proposal in which she argued that social support is "the perceived amount and adequacy of socioemotional and 
Daily Social Support and Drinking, 16 instrumental aid" (p. 148). This definition directed the researchers to diverse types (e.g., perceived, received), sources (social network members) and degrees of support received from others (appraisal). Thus, many in the field began to agree and embrace the view that social support is multifaceted and complex (Pierce, Sarason, Sarason, Joseph \& Henderson, 1996; Vaux, 1988). Consequently, Vaux (1988) introduced what he called a metaconstruct, which many in the field seemed to agree with (see Cohen, Gottlieb, \& Underwood, 2000). This metaconstruct consisted of three discrete conceptual components: perceived support (i.e. appraisals), received support (behaviors) and the social support network (i.e., resources that are made available by those with whom you have a relationship; Cohen \& Wills, 1985; Pierce, et al., 1996; Vaux, 1988). In addition, each construct can be viewed as positive or negative. Since appraisals are a subjective evaluation or understanding of a socially supportive exchange, research had deemed it a critical component when thinking about the overall social support construct (Vaux, 1988). It is through these cognitive appraisals (e.g., processes) that individuals acquire information about who is available for support, what kind of support they need, and how serious the problem may be; thus, they are a fundamental component in the social support construct.

\section{Constructs and Mechanisms of Social Support}

In researching and theorizing about the metaconstruct of social support, scholars began to differentiate received (i.e., actual support exchanges) from perceived support, frequently focusing on the latter. It is important to note that even 
Daily Social Support and Drinking, 17

though support networks are considered part of the metaconstruct, they will not be examined in this current study. Some researchers suggest that examining the quantity, quality, and the frequency of contact within the social network structure is what is needed to gain a full understanding the impact of social support on wellbeing and health (House, Umberson, \& Landis, 1988). Yet, a compelling argument has been made for the importance of looking at functions of support rather than network properties (Cohen \& Hoberman, 1983). Social networks are structural in nature, whereas perceived and received support are both more process-oriented. Further, the network characteristics do not allow researchers to separate out the mechanisms that enable perceived support to shield the individual. Thus, an examination of day-to-day exchanges that comprise support is most indicative of those support constructs. The focus of the current study, then, is on perceived and received support.

Researchers generally agreed that social support, particularly perceived social support, is associated with positive outcomes such as improved health behaviors (e.g. Cohen, et al., 2000; Sarason, Sarason, Potter, \& Antoni, 1985; Cohen, 2004). In other words, believing that others are there in times of need is linked to better health. Interestingly, research on the benefits of received support has been plagued with mixed findings (Stroebe \& Strobe, 1996). Whereas the receipt of support can positively influence some individuals on some occasions, received support can also be appraised negatively (e.g. Martire, Stephens, Druly, \& Wojno, 2002; Barrera, 
Daily Social Support and Drinking, 18

Sandler, \& Ramsay, 1981; Gleason, Iida, Bolger, \& Shrout, 2003). That is, research indicates that regardless of the support-provider's intentions, the help may not be wanted and their support may not be appraised as helpful to the recipient (DunkelSchetter, Blasband, Feinstein, \& Herbert, 1992; Cutrona, 1986).

As social support research evolved researchers began to disentangle the complex mechanisms by which social support can be further understood. Cohen and Wills (1985) presented their seminal work which identified and explained these important mechanisms, namely the direct-effect and the stress-buffering effect. The direct-effect model proposes that resources alone are advantageous regardless of the presence of a stressor. Specifically, Cohen and Wills posited that a connection with a large social network (e.g., family, friends, and coworkers) provides individuals with a sense of stability and a relatively consistent source of positive interactions. Moreover, according to the stress-buffering model, the potential negative effects of stress are reduced or eliminated as a result of resources that are provided by an individual's social network (Cohen \& Wills, 1985). It has been suggested that support influences the coping process by providing a solution or reducing the significance of the stressor (Cohen \& Wills, 1985; Cohen 2004). In the following sections I will give further develop the constructs of perceived and received support and the mechanisms through which they may operate, stress-buffering and directeffect models. 
Daily Social Support and Drinking, 19

\section{Constructs of Social Support}

The construct of social support includes two process-oriented components. In the following sections I will briefly describe and differentiate these aspects of social support; specifically, I elaborate on perceived and received support.

Perceived Support. Perceived support is based on the initial argument from Cobb (1976) that the concept of social support involves information whereby an individual considers themselves to belong, have personal value, feel cared for and loved. Higher levels of perceived support have been linked with enhanced health, self-esteem and well-being, suggesting that perceived support has a positive, direct effect on the individual (Cohen, 2004; Cohen, et al., 2000; Sarason et al., 2001).

Building on that conceptualization, others have posited that regardless of whether the individual requests help or not, the knowledge that help is available is enough to influence the impact of stressors and will assist in the coping process (Heller, Swindle Jr., \& Dusenbury, 1986). For example, Cohen and Hoberman (1983) recruited 70 college students to participate in a study examining whether the perception of support actually reduced stress. Results indicated that higher levels of perceived social support and positive life events moderated the associating between physical and depressive symptoms and life stress. Cohen and Pressman (2004) also argued that perceived support protects an individual from stressful events in distinct ways. First, just knowing that there is a support system in place, and people are there to help in times of need, may actually intervene in the stress process resulting in the 
Daily Social Support and Drinking, 20

stressor having minimal impact. In other words, the perception of the threat associated with a stressor is reduced because the individual believes that they have the resources to deal with the problem (e.g., less of a threat). In addition, they argue that perceived support may reduce the physiological reactions to the negative event or stressor and that it may thwart maladaptive and harmful behaviors. Research conducted by Lepore, Silver, Wortman, and Wayment (1996) revealed that examined women who had just lost an infant to Sudden Infant Death Syndrome found that having supportive people to talk to during traumatic and stressful times reduced the likelihood of intrusive, negative thoughts, depression and other maladaptive responses. Moreover, some research has indicated that perceived support may perhaps sedate the neuroendocrine system which results in people being less reactive to stress, while it also facilitates health-promoting behaviors such as exercise, proper nutrition, and rest (see Cohen and McKay, 1984; Cohen \& Wills, 1985; House et al., 1981).

Furthermore, research has indicated that perceived support is stable over time. Where received support (which will be discussed next) is contingent on the resources provided by others in times of need, perceived support is a more stable characteristic. Sarason and colleagues have demonstrated that individuals who have high levels of perceived support report closer, more consistent personal relationships over time (Sarason, Sarason, \& Shearin, 1986). Sarason et al. (1986) also argue that early attachment contributes to later, stable levels of perceived support. Moreover, 
Daily Social Support and Drinking, 21

they also suggest that network size contributes to the stability of perceived support over time (Sarason et al., 1986). For example, if an individual is faced with a stressor know that they have many people who can help them can reduce the effects of the stressor. Although this dissertation will not examine the influence of social networks or attachment, it is important to note the importance of those influences on perceived support.

In summary, one area of support research examines the perception (as opposed to actual receipt) of support. Many researchers find this line of research to be the central factor in understanding the impact of social support on the individual (Cohen \& McKay, 1984, Lazarus \& Folkman, 1987; Heller \& Swindle, 1983; Procidano \& Heller, 1983). This perspective is best captured by Turner et al., (1983) when they stated: "Men are disturbed not by things, but by the views which they take of things...it is after all, an axiom of social psychology that events or circumstances in the real world affect the individual only to the extent to which they are perceived" (p. 72).

Received Support. Another key construct of support, received support, has been defined as tangible support or actual support (Wills \& Shinar, 2000) and it is considered to be the specific supportive behaviors that are provided by those in an individual's support network (Sarason et al., 1990). Received support is often examined within the transactional model of stress and coping; many have argued that it is received support that makes the most significant impact on the coping process 
Daily Social Support and Drinking, 22

(Barrera et al., 1981; Hirsch 1980; Gottlieb, 1984). Specifically, Gottlieb (1984) suggests that perceived support assists in the primary appraisal process; however, researchers need to recognize that this psychological response to stressors may not be enough when actual coping behaviors are required. In other words, when faced with a stressor, having to regulate emotions and having to attend to the tension of the stressor may require more than perceived support. The actual tangible support provisions that are made available by those in a support network may be what are required to engage in positive coping strategies.

Gottlieb (1984) goes on to argue that in early social support research, investigators were not examining the explicit behaviors that comprised received support. Consistent with this line of reasoning, Barrerra and colleagues suggested that to understand social support, one must consider natural helping behaviors (Barrera et al., 1981; Hirsch 1980). Moreover, Barrera and Ainlay (1983) conducted research using a sample of college students in an attempt to further understand and define received support. The students were asked about the process of receiving help from her or his natural support providers; consequently, analyses resulted in a four factor model of received support types, consisting of directive guidance (offering advice and feedback), nondirective support (intimacy, availability, esteem, and trust), positive social interaction (hanging out), and tangible assistance (physical or financial help). Interestingly, Barrera and Ainlay (1983) concluded that the frequency of all types of received support was associated with higher levels of 
Daily Social Support and Drinking, 23

perceived support and higher reported levels of family cohesiveness, suggesting that how a person appraises the process of receiving support is associated with positive outcomes and that there is a link between perceived and received support.

Yet, not all research examining received support has revealed benefits of such support (Stroebe \& Strobe, 1996). Some research has suggested that received support can also be appraised negatively resulting in feelings of dependence and reduced well-being (e.g. Barrera et al., 1981; Gleason et al., 2003; Martire et al., 2002; Reinhardt, Boerner \& Horowitz, 2006). Interestingly, the intent of the support provider may be positive but individuals who are the recipients of support can misconstrue the motivation of the support provider. Moreover, the individual may not want support no matter what the intensions are, which may result in an increase in the perceived intensity of the stressor. As an example, Davidowitz and Myrick (1981) found that people who were grieving because they had lost a loved one did not find statements such as "You shouldn't question God's will," and "Be thankful you have another son" as helpful. Similarly, Wortman and Lehman (1985) reported in their study on coping with the death of a family member, such as a child or spouse, that many individuals attempted to offer social support by making statements such as: "I know exactly how you feel," which the recipients found to be unhelpful and negative. Received support can also be viewed negative because individuals feel as though it is excessive helping or overinvolvement by others. Although support providers are trying to help and they think they are being of assistance they, in fact, 
Daily Social Support and Drinking, 24

may make the support recipient feel guilty, or as if they are lacking autonomy and competence (Coyne, Wortman, \& Lehman, 1988; Rook, 1984). Thus, it is imperative that the support being provided is positively appraised.

\section{Mechanisms of Social Support}

As briefly described earlier, research has suggested that independent of the perceived and received distinction there has been research on the mechanisms by which social support functions (Cohen \& Wills, 1985; House et al., 1981). In an extensive review of previous studies, Cohen and Wills (1985) posited that there was a relationship between social support and health and well-being. More specifically, they were interested in whether this relationship between support and well-being was due to a direct-effect of social support on well-being or was it due to the stressbuffering qualities of social support. Interestingly, Cohen and Wills (1985) found that there was evidence consistent with both the direct-effect model and the stressbuffering model of social support. They found that both models are similar, yet are functionally different. More specifically, the direct-effect model of support postulates that having relationships has a beneficial effect on well-being. The hypothesis is that being socially integrated allows an individual to have higher levels of perceived and received support, which results in well-being (Cohen \& Wills, 1985; Rodriguez \& Cohen, 1998). In analytic terms, the focus of this model is on the main effect of relationships on health and well-being. In contrast, the stress-buffering model of social support posits that support is advantageous for health and well-being 
Daily Social Support and Drinking, 25

because it protects individuals from possible deleterious effects of stress (Cohen \& Wills, 1985; Rodriguez \& Cohen, 1998). Whether the support is in fact provided or an individual simply perceives that it will be there in times of need, stress may be reduced in both the short term and the long term. In addition, after examining much of the research, Cohen and Wills (1985) concluded that buffering can take the form of perceived or received support as depicted in Figure 2. The ensuing sections will explain both mechanisms more specifically.

Stress-buffering model. One mechanism through which social support operates is described by the stress-buffering model. This model proposes that social support can protect an individual from the potentially harmful effects of stressors (Cohen \& Wills, 1985; Rodriguez \& Cohen, 1998). By boosting an individual's perceived ability to effectively and efficiently cope with any potential demands; Cohen and Pressman, 2004; Thoits, 1986). The damaging effects of stressors are moderated by an individual's capability to appraise the stressor as less threatening; and the perceived level of support that one has regarding their social network plays a vital role in this appraisal process (Cohen \& McKay, 1984).

Social support also influences the stress process by offering a solution or reducing the perceived significance of a stressor (Cohen 2004; Cohen \& Wills, 1985). Further, when stressors are encountered, individuals' certainty that they have the resources (i.e., high levels of perceived support) that they may need to deal with the stressor will reduce the negative effects of stressors thus enabling them to take 
Daily Social Support and Drinking, 26

action (Cohen, et al., 2000; Cohen \&Wills, 1985, Cutrona, 1986; Thoits, 1986).

Furthermore, once an individual has experienced a stressor individuals may draw on their socially supportive network in a number of ways; for example, they may request information and advice, distraction, or to have someone that will listen to them vent (Cohen \& Wills, 1985). The key for this type of support to work is that it must match the need of the recipient and be positively appraised. At that time, support may reduce the effects of the stressor by providing tangible assistance, alternative solutions, or providing a distraction to the person in need (Bolger, Zuckerman, \& Kessler, 2000; Cohen \& Wills, 1985; Cohen et al., 2000). This idea is appealing but it is important to note that the person receiving social support must appraise that support as helpful and appropriate; in other words, positively appraised support is the key.

Direct effect model. The direct effect model of social support proposes that having relationships with others and being socially integrated has a positive and direct effect on an individual's well-being independent of one's stress. These relationships offer individuals the opportunity to have positive interpersonal interactions, opportunities for social roles (e.g., friend, confidante) and vital feedback such as feedback on health behaviors (e.g., not smoking; exercising; proper diet; Rodriguez \& Cohen, 1998).

Social support directly affects an individual by serving as a protective function as well as a preventative function. Social support can influence an 
Daily Social Support and Drinking, 27

individual in such a way that it prevents the occurrence of some stressful events. For example, students who study together will get a better grade which, in turn, affects well-being. Wheaton (1985) describes this through his "stress deterrent model" in which he suggests that support can prevent the experience of stress. Heller et al. (1986) argue that social resources assist with social adjustment and adaptation which results, in individuals viewing themselves with high esteem, which in turn, results in them appraising fewer events as threats and more events as challenges.

Researchers have also contended that through social integration and connectedness individuals are able to receive an assortment of dependable and consistent resources; moreover, they are able to obtain vital health and social information from those in their social network (Cohen \& McKay, 1984). For example, when an individual is given information from someone in their social network that helps them be more capable of negotiating new roles, as well as, how to meet the required expectations within the current roles that they hold, they will feel a greater sense of belonging and self-worth compared to those to do not have a social network that offers such valuable information (Cohen, 2004; Cohen \& Wills, 1985; Thoits, 1983).

As social support research progressed, some researchers began to probe the relationship between social support and health. For example, Cohen (2004) reported that in a study that used social integration (i.e., social support) as a predictor of health and other variables, researchers found that those participants who were more 
Daily Social Support and Drinking, 28

socially integrated lived longer than those who were less socially integrated (see Berkman \& Syme, 1979). This study suggests that having supportive individuals in one's life will have a direct effect on mortality. Moreover, researchers have found that, although social support is associated with many positive outcomes, men tend to profit more from social support than women (House, et al., 1988). It is possible that women pay a price for the quality of their relationship more than men (Coriell \& Cohen, 1995) or that women feel more obligated to those in their social network than men, thus, have more stress because of their social networks than men (Kessler \& McLeod, 1985). However, it is important to remember that although being socially integrated is more demanding and stressful for women, men and women both benefit significantly because these relationships.

\section{Summary of support mechanisms}

Perceived support, which has been the dominant focus in the research literature has both a direct and buffering effect on health and well-being. Specifically, the direct effect (i.e., main effect) of perceived support involves primary appraisals whereby individuals experience the receipt of support, or perceive that they have available resources, which directly enhances health behaviors and well-being. Perceived support also provides stress-buffering wherein individuals believe that resources are available if needed which reduces the effects of the stressors. Essentially, stressors become less threatening because individuals understand that support is available. 
Daily Social Support and Drinking, 29

Received support, on the other hand, has a direct effect on the individual because this type of support directly affects an individual's health and quality of life through tangible, informational, informational, or companionship support on a relatively regular basis (Wills \& Shinar, 2000). Previous research suggests that constant and reliable sources of support are an important factor in health and wellbeing; some argue that it fulfills a basic need that all humans have (Baumeister \& Leary, 1995, Bowlby, 1969). Further, received support in times of stress gives individuals tangible resources to deal with problems, which in turn reduces the negative effects of stressors. To find supportive evidence for buffering, it is necessary to consider the extent to which supportive exchanges (e.g., positively appraised received support) moderate the stressor-outcome association.

\section{Social Support and Health Behaviors}

Over time there has been a remarkable amount of research focused on the possible positive effects of support on health and well-being (e.g., Broadhead, Kaplan, James, Wagner, Schoenbach, Grimson, Heyden, Tibblin, \& Gehlback, 1983; Cohen, et al., 2000; Cohen \& Syme, 1985). Interestingly, some research has found that social support is not always associated with better health and well-being (Cohen \& Wills, 1985), which lead some to examine specific health-related areas in which support may or may not be beneficial. Some broad areas of interest have been the relationship between social support and health supporting behaviors such as effective emotion regulation and better self-care (e.g., Cohen, 2001; Helgeson, Cohen, Schulz, 
Daily Social Support and Drinking, 30

\& Yasko, 2001; House et al., 1988); as well as illness related research such as cancer and susceptibility to colds, to name a few (e.g., Cadzow \& Servoss, 2009; Cohen \& Lemay, 1997; Cohen, Kaplan, \& Manuck, 1994; Dunkel-Schetter, 1984). Other areas of interest have included psychological stress (Handey \& Chassin, 2008; Mulia, Schmidt, Bond, Jacobs \& Korcha, 2007; Pierce, Frone, Cooper \& Russell, 1996), mental health (Coyne \& DeLongis, 1986; Kawachi \& Berkman, 2001; Schuster, Kessler, Aseltine, Jr., 1990) and health-compromising behaviors such as smoking and drinking (Chi, Kaskutas, Sterling, Campbell \& Weisner, 2008; Groh, Jason, Davis, Olson, \& Ferrari, 2007; Handey \& Chassin, 2008; Mulia, Schmidt, Bond, Jacobs \& Korcha, 2007; Pierce, et al., 1996; Wills, Resko, Ainette \& Mendoza, 2004; Windle \& Windle, 1996). This dissertation will examine alcohol consumption in relation to social support.

Most of the research that has examined social support effects on drinkingrelated outcomes has focused on the association between support and alcohol problems, particularly among high risk populations (alcohol dependent, abusers or adolescents). For example, one study demonstrated that individuals who have higher levels of social support and also report more subjective well-being and generally have better post-treatment outcomes (e.g., Beattie, Longabaugh, Elliott, Stout, Fava \& Noel, 1993). Conversely, those who have less supportive relationships tend to have more problems in recovery (e.g., Humphreys, Moos \& Finney, 1995). Similarly, Groh et al., (2007) found that in a sample of individuals who were living 
Daily Social Support and Drinking, 31

in an abstinent-communal living setting, social support was directly related to alcohol use. Specifically, general support from friends and family predicted less alcohol consumption (Groh et al., 2007). Furthermore, they put forth that it is necessary for individuals that have alcohol dependence to have highly supportive networks if they are to continue to abstain from drinking (Groh et al., 2007).

While researchers have examined the biological and physiological benefits of moderate drinking very little effort has gone in to examining the psychosocial costs and benefits of moderate drinking in normative samples. Pierce and colleagues argued that the much of the research that has examined psychological measures, such as stress and drinking, have used samples that were so unique (i.e., arthritic patients, depressed patients, alcoholic patients) that generalizing to a normative sample was not possible (Pierce et al., 1996). Furthermore, Pierce et al. (1996) argued that future research should examine different types of drinking behaviors and motives. In the next section I build on this view and I will present reasons why it is important not to categorize all drinking behaviors together, especially when examining social support. 
Daily Social Support and Drinking, 32

\section{CHAPTER 3}

\section{Alcohol Consumption}

Drinking behaviors are complex and multidimensional and comprise not one but several distinct behavioral types (Cooper et al, 1995; Cooper, Russell, Skinner \& Windle, 1992b; Mohr et al., 2001). It could be argued that to fully understand the relationship between social support and alcohol consumption, researchers must disentangle these specific behaviors. Cox and Klinger (1988) and Cooper (1994) theorized that distinct drinking behaviors are guided by two primary principles; first, people drink in order to achieve some outcome that is important or valued and second, people are motivated to drink because of unique antecedents and consequences that attend to different needs or functions (Cooper, Russell, Skinner, Frone, \& Mudar, 1992a; Cooper, 1994; Cooper, Russell, \& George, 1988, Cox \& Klinger, 1988). For example, in some instances people are motivated to use alcohol as their chief method of dealing with psychological tension because they are unable to use more adaptive coping strategies, such as seeking social support. Consequently, this maladaptive drinking pattern becomes a permanent way to cope (Cooper, 1994; Cooper, et al., 1988). Conversely, others are motivated to drinking in social situations; which is not considered so long as drinking levels are not too high. Moreover, social drinking is not associated with coping processes but rather it is linked to social enhancement and positive mood (Cooper et al., 1992ab; Cooper, 1994). To fully understand drinking behaviors, it is appropriate to examine the 
Daily Social Support and Drinking, 33

motivations that influence behaviors. The next section will elucidate Cooper's (1994) 4-factor model of drinking motives to offer a framework by which drinking behaviors can be better understood.

\section{Drinking Motives}

People drink for a variety of reasons, and researchers have attempted to disentangle what these reasons, or motivations, might be. As previously mentioned in the introduction, Cox and Klinger $(1988,1990)$ argued that drinking motives can be distinguished by two dimensions: the valence (positive or negative) and source (internal or external) of the outcomes (Cooper, 1994). Building on that notion, Cooper presented a four-factor model alcohol consumption that consists of internal and positive reinforcement motivation, external and positive reinforcement motivation, external and negative reinforcement and, the most problematic drinking behavior, drinking as a method of coping which is considered internal and negative reinforcement. Moreover, it was this drinking-to-cope motivation (e.g., tensionreduction drinking behaviors) that was linked with the most detrimental consequences (e.g., abuse, dependence; Cooper, 1994; Cooper et al., 1995). In sum, there are distinct drinking behaviors with different antecedents and consequences. Drinking as a method of coping is the maladaptive one that is most closely linked to social support processes.

What is more, research has found that drinking for social reasons is normative and has not been linked to coping, social skill issues or drinking problems 
Daily Social Support and Drinking, 34

unless they drink too much (e.g., binge drinking; Cooper, et al., 1998). More specifically, Cooper et al. (1992b) found that socially motivated drinking behaviors were considered to be more normative across race and gender groups. Moreover, those who drank for social reasons did not show a significant increase in drinking or drug problems compared to those individuals who endorsed drinking-to-cope motives.

Cooper et al. (1995) also found that each drinking motive was correlated with specific drinking contexts. Specifically, she found a significant association between drinking as a method of coping and drinking at home (e.g., solitary context); whereas those who drank to enhance their mood and experiences were more likely to drink in social settings (e.g., bars, clubs). This suggests that different behavioral motivations occur in different contexts. In other words, why people drink and where they drink go hand-in-hand. Accordingly, it is critical to take context in to account when examining drinking behaviors and motivations and to create an accurate and complete multidimensional model of alcohol consumption.

Given the problems associated with drinking to cope, I contend that the drinking behavior that is most closely linked to drinking problems and social support is drinking as a method of coping (i.e., tension-reduction drinking); or more specifically, solitary drinking at home. Cutter and O'Farrell (1984) revealed that solitary drinking at home was associated with a variety of negative personal reasons for drinking. More specifically, individuals were likely to drink alone as a way of 
Daily Social Support and Drinking, 35

coping and forgetting that they were "not the kind of person" they wanted to be or to become less aware of the judgment of others (Cutter \& O'Farrell, 1984). Rather than turning to others in times when they felt bad about themselves (e.g., seeking out social support), those individuals drank alone. To further elucidate the concept of tension-reduction drinking, the next section will offer a detail review of the tensionreduction drinking literature and theories on which these models have come to be understood and how it might pertain to social support.

\section{Tension-Reduction Drinking}

The notion that people are motivated to drink as a method of coping and reduce the impact of negative experiences is derived from the tension-reduction hypothesis which was presented by Conger in 1956 . He posited that people drink to reduce anxiety and stress caused by negative experiences and situations, and the reduction in tension that is attained due to the alcohol consumption reinforces the drinking behavior. Although this view gained much interest in the field over time, research was plagued with inconsistent findings. While the research that was reviewed by Cappell and Herman prior to 1972 did not yield significant support for the TRH, the mid 1970's brought a new interest in TRH. During this period, researchers employed more tightly controlled experiments as a way to examine the two basic tenets of TRH: first, alcohol reduces psychological tension, and secondly, people drink alcohol because it reduces tension (Greeley \& Oei, 1999). It is important to note that this dissertation only addressed the second tenet, as it is the 
Daily Social Support and Drinking, 36

most directly relevant to support processes (i.e., does social support reduce the extent to which people drink at times they are stressed).

In the 1980's and 1990's researchers began recognizing that the concepts of the tension-reduction drinking model were too broad to be useful (Greeley \& Oei, 1999); thus, a number of important theories and theoretically based models surfaced. These specific models offer researchers a way to further understand tensionreduction drinking behaviors. Such models include: alcohol myopia theory, selfawareness model and social learning theory.

One of the most prominent theories on the effects of alcohol consumption on behavior is alcohol myopia theory (Sayette, 1999; Steele \& Josephs, 1990). In this model, Steele and Josephs (1990) argue when people drink they can only focus on one thing at a time; thus, ruminating about a stressor is difficult if a person is distracted. Steele and Joseph (1990) also posit that if an individual is not engaged in a distracting situation that keeps their mind of negative self-focused thoughts then the consequences of alcohol myopia may be very negative and damaging.

Complementary to alcohol myopia theory Hull's self-awareness model (1981), which hypothesizes that alcohol can be used as a method of coping by reducing psychological tension through lessening of painful personal self-awareness. In particular, after a negative or failure experience an individual may drink to avoid negative self-evaluation that typically follows failure (Hull, 1981). Hull and colleagues examined this process by conducting an experiment in which they had 
Daily Social Support and Drinking, 37

participants consume alcohol and then describe what they liked or disliked about their physical appearance. As predicted, those who consumed alcohol had fewer selffocused comments and statements when compared to the control group (Hull, Levenson, Young, \& Sher, 1983). The researchers argued that drinking alcohol hampers higher-order encoding processes (see Hull \& Levy, 1979) resulting in reduced self-awareness. In other words, the individuals who consumed alcohol had fewer self-focused statement than those who did not consume alcohol. Hull and colleagues (1983) suggested that the self-awareness model does not account for all drinking behaviors but does offer researchers insight on "everyday patterns of alcohol use" (p. 796).

Further, Bandura (1969) applied social learning theory to understand drinking behaviors and more specifically drinking as a method of coping (e.g., tensionreduction drinking). He posited that to some degree alcohol problems were due to a person's lack of coping skills during times of stress. More specifically, Bandura (1969) suggested that there are interactive relationships between individuals and their environment and these associations influence behavior over time. He called this "reciprocal determinism.” Building on Bandura's work, later research found that individuals who drink as a method of reducing stress or psychological tension are unlike others in that they tend to use maladaptive coping strategies, such as avoidance behaviors. Moreover, these same individuals tend to believe that alcohol will positively affect their mood and reduce stress. In other words, they have high 
Daily Social Support and Drinking, 38

alcohol-related outcome expectancies (Abrams \& Niaura, 1987; Cooper, et al., 1988; Maisto, Carey, \& Bradizza, 1999).

In sum, theories and models have demonstrated that individuals use alcohol to regulate negative emotions (Cahalan, Cisin, \& Crossley, 1969; Cooper et al., 1988; Mulford \& Miller, 1963). Whether it is to reduce self-awareness or to not think about the problem at all; alcohol is often used as a coping tool. For instance, Farber, Khavari, and Douglass (1990) found in a cross-sectional study to determine reasons for drinking they found that $93 \%$ of the individuals who were diagnosed as being alcoholics reported being escape (i.e., tension-reduction) drinkers. Other studies suggest that people may drink as a method of coping because they feel that they have no other coping alternatives; thus alcohol becomes the main method of coping. For example, an experimental research study conducted by Marlatt, Kosturn, and Lang (1975) revealed that individuals who were unable to retaliate against a confederate who provoked them were likely to drink significantly more than those who were able to retaliate. Marlatt and colleagues argued that alcohol was used as a method of coping in these situations because no other method of coping was available (Marlatt et al., 1975).

In terms of the types of stress most likely to trigger drinking to cope, research has revealed that interpersonal stressors are particularly detrimental. Higgins and Marlatt (1973) have conducted a series of studies in which they were able to demonstrate that social stressors are especially meaningful to most people and 
Daily Social Support and Drinking, 39

significantly more meaningful than physical stressors. In fact, they found that when individuals were faced with an interpersonal stressor they drank significantly more than those who were not exposed to an interpersonal stressor (Higgins \& Marlatt, 1973). These results are not surprising given that research conducted by Bolger and colleagues indicated that interpersonal stressors are some of the most damaging to an individual's health and well-being (Bolger, DeLongis, Kessler, \& Schulling, 1989; Caspi et al., 1987). Bolger and colleagues found that people reported that negative interpersonal experiences were appraised as the most negative and the most frequent of daily stressors (Bolger et al., 1989). Although major life events have been associated with depression and other psychological issues (Brown \& Harris, 1978) daily stressors have been implicated in physical issues and problems (Bolger et al., 1989; Caspi, et al., 1987). For that reason in this study the variable of "stress" will be operationalized as daily negative interpersonal exchanges. Moreover, Mohr et al. (2001) examined drinking and negative interactions and found a significant association with daily negative interpersonal interaction and solitary drinking at home. Since this study will be focusing on daily solitary drinking at home, examining specific stressors (such as negative exchanges) is consistent with previous research and will be contributing to the field.

In addition, Bolger et al. (1989) argued that researchers should focus on daily interpersonal exchanges rather than large, global events or retrospective accounts; it is in these daily events that researchers are able to disentangle the stress and coping 
process (a point I will return to in the next chapter). With regards to social support, Caspi et al. (1987) pointed out that immobilizing social support may buffer an individual from the detrimental effects (e.g., solitary drinking at home) of daily stressors. Therefore, it is reasonable to suggest that using a daily process approach to examine social support and negative exchanges may offer more insight than relying on retrospective reports. In addition and importantly, Mohr et al. (2001) pointed out that tension-reduction hypothesis describes a within-person relationship (i.e., do people drink when they are stressed). Accordingly, when considering exchanges and tension-reduction drinking it is important to use the appropriate methodology when investigating the important processes. In other words, when examining within-person phenomena it is critical to examine it using daily process methodology, a topic that I turn to now in Chapter 4. 
Daily Social Support and Drinking, 41

\section{CHAPTER 4}

\section{Daily Process Methodology}

The use of daily process methods has been important to the stress, social support and alcohol consumption literatures. Although the majority of research in these areas has used traditional cross-sectional and on occasion, multi-wave longitudinal methods of data collection, researchers are becoming more interested in the within-person processes in alcohol consumption (Armeli et al., 2005; Mohr et al., 2001; Park et al., 2004) and social support (Coyne \& DeLongis, 1986; Newsom, Nishishiba, Morgan, \& Rook, 2003). The flexibility in daily process methods makes them exceptionally appropriate to capture information on daily variability in alcohol consumption and stress, as well as supportive interpersonal interactions.

There are three methods by which researchers record daily processes (e.g., events and experiences), time-contingent, signal-contingent and event-contingent designs (Bolger, Davis, \& Rafaeli, 2003; Reis \& Gable, 2000; Wheeler and Reis, 1991). Each of the three methods offers specific strengths and weakness and the decision to use one method over the other would depend on the specific goals of a study. Event-contingent recording requires participants to complete a survey or record information about a specific event or behavior (Bolger et al., 2003; Reis \& Gable, 2000) such as when they drink or have a negative exchange. One serious issue with this design is that it can be rather rigorous for participants; therefore, it is important for researchers to have a well-defined description of the events and 
Daily Social Support and Drinking, 42

behaviors that are to be recorded (Bolger, et al., 2003). Research using time-based designs typically has a primary goal of examining events or experiences that are ongoing (e.g., moods) and that can be captured and evaluated during the course of a specific period of time (e.g., day, week). Researchers often obtain information by either asking participants to engage in an end-of-day survey (e.g., bedtime diary) or during predetermined time points throughout the day. Daily survey times can be fixed, random or a combination of fixed. Finally, signal-based designs are rather straightforward in that the participants are asked to record data after they are alerted often at random time points throughout the day. Bolger et al. (2003) points out, researchers do not have to choose one design over another; it is possible to design a study where multiple types of recordings can take place. For some researchers it makes sense to combine the types of designs. For instance, Mohr et al. (2001) instructed 100 participants who were recruited from the local community, to record alcohol consumption as it occurred (event-contingent) on a handheld computer throughout the day. At the end of each day they asked participants to record their interpersonal exchanges in a bedtime diary. The data collected by Mohr and colleagues offered insight to daily drinking behavior, drinking contexts (e.g., home alone or away from home) and the influence of daily positive and negative interpersonal exchanges (Mohr et al., 2001). However, because the interpersonal exchanges were only recorded once per day it was not possible to establish temporal within-day relationships between exchanges and drinking. 
Daily Social Support and Drinking, 43

Using daily process methods researchers are able to differentiate within- and between-person processes. For example, DeLongis, Capreol, Holtzman, O’Brien, and Campbell (2004) found that couples with higher levels of marital satisfaction reported more daily social support and less same-day negative affect. Multilevel techniques allowed for between-person variation in within-person relationships, also known as cross-level interactions, to be assessed. In other words, moods, support and strain were examined as they unfold over the course of the study (i.e., daily; withinperson); while marital satisfaction was compared between couples. DeLongis et al. (1988) argued that using daily process (i.e., within- and between-person) methods enables the researcher to examine very different, and important, questions. With this in mind, using daily process methods in conjunction with multilevel modeling to probe the moderating effects of daily social support on the stress-drinking relationship would be ideal. Later in the chapter I elaborate on using daily process methods to examine the stress-drinking relationship.

\section{Daily Process Methodology and Social Support}

Researchers are becoming increasingly interested in examining the withinperson association between social support and stress (DeLongis, et al., 1988; Pierce et al., 1996). Indeed, the social support processes posited by some theorists are within-person processes; meaning that to fully understand social support researchers must examine the idiographic processes of support over time (DeLongis et al., 2004). DeLongis and colleagues put forth that these types of methods reduce recall error 
Daily Social Support and Drinking, 44

and allow for close inspection of temporal associations (DeLongis et al., 2004). Although the amount of research that has focused on social support as a withinperson process has been minimal (DeLongis et al., 2004); the results of this research has shed light on the impact of daily support.

Cutrona (1986) was one of the first in the social support field to use process methodology to understand social support at a microanalytic level rather than from a retrospective point of view. Cutrona (1986) argued that to understand social support it is critical to understand the effects that daily interpersonal experiences have on support. Her daily diary study demonstrated a significant association between perceived social support and the amount (frequency) of helping behaviors that an individual reported. Cutrona (1986) was able to determine that help from others was particularly salient when reported immediately after a stressful event; also, perceived support predicted socially supportive behaviors only at times when the participants also reported at least one stressor; which is consistent with the stress-buffering hypothesis (Cohen \& Wills, 1985).

Although studies have investigated the direct-effect model of social support few have examined it using daily process methodology. What research has been done has generally focused on mood as an outcome, which is a key component in understanding overall well-being (Diener, 1984) and is an implied mediator in the stress-drinking relationship (e.g., Armeli et al., 2005; Hussong, Galloway \& Feagans, 2005; Park, et al., 2004; Todd et al., 2003; Todd, Armeli, Tennen, Carney, Ball, 
Daily Social Support and Drinking, 45

Kranzler, \& Affleck, 2005). In one study examining the direct-effect of social support on mood, Feldman and colleagues sampled chronic pain patients and found that those who reported receiving social support ${ }^{1}$ the previous day had lower negative mood (Feldman, Downey, Schaffer-Neitz, 1999). Like many other social support studies, this study did not probe positive mood-social support associations but the results were consistent with the direct-effect model in that social support had a main effect on mood. They also examined the interaction of support and pain; results indicated that social support had a buffering effect on pain. Further, Vittengl and Holt (1998) found that daily social support directly enhanced positive mood. Consistent with this, Gable, Reis, Impett, and Asher (2004) examined daily exchanges and mood, finding that on days in which individuals reported sharing a positive event with others (one form of positive exchanges), they experienced significantly higher positive mood and life satisfaction.

A number of daily process studies have also corroborated aspects of the stress-buffering model. In their study of married adults with a chronic pain disorder, Feldman et al. (1999) found that those with higher levels of perceived social support reported lower levels of negative mood following a painful day; in other words, support moderated concurrent effects of pain on overall negative mood. Caspi et al. (1987) also found that perceived levels of social support (conceptualized as network

\footnotetext{
${ }^{1}$ Although Feldman et al. (1999) termed it perceived support, they were describing activities wherein a person recorded the occurrence of a helping behavior. This is most consistent with the concept of received support in the present and most other studies.
} 
Daily Social Support and Drinking, 46

members available in times of need) moderated the effect of stress on the next day's mood.

In sum, studies are beginning to examine the relationship between social support and health outcomes using daily process methodology. This rather new innovative technique has allowed researchers to examine the temporal relationships between support and other health-related variable without issues of retrospection biases and error.

\section{Daily Process Methods and Alcohol Consumption}

Daily process methods have offered new insights in to tension-reduction drinking motivations and behaviors; just as with social support literature, this method has allowed researchers insight on daily behaviors unlike before. While some have argued that traditional designs (i.e., cross-sectional, longitudinal designs) do not capture the fluctuating drinking processes. These issues have been addressed with the advent of daily process methods.

Another clear advantage of daily process methods is that it can reduce issues that come with asking people to recall the past. Interestingly, when considering recall of alcohol consumption, research has found that there is a rather small window in which a participant will record accurate information. Perrine, Mundt, Searles, and Lester (1994) asked participants to report daily drinking and give concurrent breath and saliva samples each night for 28 days. Perrine and colleagues found significant correlations between the physical and retrospective data $(r=.72)$; thus deeming 
Daily Social Support and Drinking, 47

recall of drinking valid and reliable as long as the information was gathered within 24 hours after consumption (Perrine et al., 1994). In sum, to obtain accurate accounts of alcohol consumption it is necessary for participants to record the information within 24 hours of consumption. Moreover, if researchers want to examine mood or interpersonal event-related drinking then daily process methods are necessary (Hussong, 2007; Mohr et al., 2005; Swendsen, Tennen, Carney, Affleck, Willard, \& Hromi, 2000).

A concern with daily process studies is possible participant reactivity, but research suggests that reactivity is generally minimal. Hufford and colleagues found that when asked, participants stated that reporting their daily drinking over the course of two weeks did not cause them to drink more or less (Hufford, Shields, Shiffman, Paty \& Balabanis, 2002). Real-time monitoring supported this claim (Hufford, et al., 2002). Generally, studies that examine drinking over a longer period of time (e.g., 48 weeks) have shown modest participant reactivity (Collins, Morsheimer, Patty, Gnys, \& Papandonatos, 1998; Litt, Cooney, \& Morse, 1998)

While many studies have examined social support, alcohol consumption, and stress (see Groh et al., 2007; Handey \& Chassin, 2008; Mulia, Schmidt, Bond, Jacobs \& Korcha, 2007; Pierce, et al., 1996) no studies have examined the relationship between daily received support and alcohol consumption via direct and stress-buffering mechanisms. In a similar study, Mohr and colleagues used daily process and found that on days when individuals experienced more negative 
Daily Social Support and Drinking, 48

exchanges they were more likely to engage in solitary consumption compared to those who had less negative exchanges (Mohr et al., 2001). Conversely, when the individuals experienced more positive exchanges they were more likely to drink away from home with others. In another related study, researchers found that college students drank more on days when they experienced more negative social contact and more negative moods (Mohr et al., 2005). Interestingly, only one study, to my knowledge, has examined this relationship by examining the impact of social support, daily stress and drinking using an adult sample of individuals who have not been diagnosed as alcohol dependent or abusers using daily process methodology. In this study Wang, Liu, Zhan, and Shi (2010) argued that daily stress can have serious negative consequences such as maladaptive coping (e.g., tension reduction drinking), decrease in job-related self-image and an increase in work or family problems; moreover, they argue that social support may serve as a buffer. Using a Chinese sample, Wang et al. (2010) specifically examined the possible moderating effects of social support on work/family stress and tension-reduction drinking behaviors. After collecting daily data for 5 weeks, they found that coworker support (between-person) moderated the work-to-family conflict- and family-to-work conflict-alcohol use (within-person) relationship. In other words, social support moderated the daily stress-drinking relationship.

This dissertation built on Wang et al.'s (2010) work in a number of ways. First, this dissertation examined drinking context as well as drinking behavior. As 
Daily Social Support and Drinking, 49

mentioned in Chapter 2, to fully understand drinking behaviors it is critical to examine the contexts in which they are taking place (Cooper et al., 1992a; Cooper, 1994; Mohr et al., 2001; Mohr et al., 2005); thus, that is what this project set out to accomplish. Moreover, in an attempt to capture the stress-drinking relationship as it unfolds throughout the day, this dissertation used data that was collected multiple times each day rather than once per day. This reduced retrospection error and recall bias which is critical when examining a psychological process (Bolger et al., 1989). Unlike the Wang et al. (2010) study, this dissertation used a sample comprised of men and women from the local community. 
Daily Social Support and Drinking, 50

\section{CHAPTER 5 \\ Purpose and Hypotheses}

Previous research has revealed specific behaviors associated with tensionreduction drinking (drinking-to-cope) such as solitary drinking (Conger, 1988) yet it has only been relatively recently that researchers have attempted to disentangle the effects of daily experiences on these possible deleterious behaviors (see Mohr et al., 2001). With this in mind, the goal of this dissertation is to further understand how daily social support may have a direct and a stress-buffering effect on tensionreduction drinking behaviors; more specifically, drinking at home alone. Moreover, from this point forward, solitary drinking is operationalized as drinking home alone and drinking at home not interacting with others.

This dissertation uses data from a larger study in which daily alcohol use and emotion regulation was probed. The 49 individuals who were moderate-to-heavy drinkers agreed to carry a handheld interviewer for 30 days and three times each day (late afternoon, evening) the handheld interviewer would alert the participant to take a 2-3 minute survey. Each survey probed interpersonal exchanges and drinking behaviors. A benefit of this type of methodology was that it allowed me to examine the temporal associations of the variables of interest while reducing retrospection bias. In other words, I was able to capture the social support-later drinking relationship process as it unfolds. Notably, with these data I am able to examine lagged associations; for example, how daily negative interpersonal exchanges predict 
Daily Social Support and Drinking, 51

subsequent within day drinking; and how social support moderates this relationship. This allows for a closer examination of daily variability of support and drinking behaviors. Hypotheses

Received Social Support. Received social support is examined within the direct-effect and stress-buffering models of social support. More specifically, the direct-effect model of social support posits that receiving stable, positive, social support has a direct effect on health and well-being (Cohen \& Wills, 1985). Moreover, research suggests that individuals who have more social support are more likely to engage in more positive health behavior and less likely to engage in risky or maladaptive behaviors such as drinking alone (Cohen, 1988; Cohen et al., 2000). Thus, I hypothesize that at times when individuals report more positively appraised socially supportive interpersonal exchanges, they will also report less subsequent drinking at home alone relative to times in which they report fewer positively appraised received support (see Figure 5).

$H_{1}$ : I predict that, consistent with the direct effect model, daily supportive interpersonal exchanges (SIE) will be negatively associated with subsequent drinking at home alone. Specifically, at times when people report more positively appraised socially supportive exchanges, they will report less drinking at home alone in the next time period relative to times when they report fewer positively appraised supportive exchanges. 
Daily Social Support and Drinking, 52

Stress-Buffering Model. The stress-buffering model of social support suggests that received support can reduce the impact or even negate the effects of stressors on mental and physical health (Cohen et al., 2000). When stressors are encountered, the certainty of having available resources (e.g., perceived support), as well as receiving tangible support resources (i.e., received support), is hypothesized to lessen the negative effects of stressors (Cohen, et al., 2000; Cohen \&Wills, 1985, Cutrona, 1986; Thoits, 1986). In a sense, the buffering effect of social support works to disrupt drinking-to-cope motives and offers resources that an individual needs during stressful times (Pierce et al., 1996). Based on this model, at times when individuals experience an increase negative interpersonal exchanges and higher levels of positively appraised daily social support, they will also report less later drinking at home alone relative to days when they have lower levels of socially supportive exchanges (see Figure 5).

$\mathrm{H}_{2}$ : Daily supportive exchanges will moderate the negative exchangesubsequent drinking at home alone relationship. The negative interpersonal exchange-subsequent (next time period) drinking at home alone relationship will be stronger at times with fewer socially supportive exchanges relative to times with more socially supportive exchanges.

This hypothesis examines how daily social support will moderate the negative exchange-later drinking association. More specifically, I predict that at times in which individuals report more negative exchanges and socially supportive 
Daily Social Support and Drinking, 53

exchanges they will also report less subsequent drinking at home alone relative to times when they have less social support.

Perceived Social Support. Similar to daily received social support, previous research suggests that perceived support is directly associated with positive health behaviors (Cohen \& Wills, 1985). In addition, higher levels of perceived support have been linked with enhanced health behaviors, suggesting that perceived support has a positive, direct effect on the individual (Cohen, 2004; Cohen, et al., 2000; Sarason et al., 2001). Thus, this dissertation examines the effects of perceived support on solitary drinking at home behaviors (see Figure 6).

$H_{3}$ : Individuals with higher levels of perceived social support will report less drinking at home alone throughout the course of the study compared to those with lower perceived social support.

In $H_{4}$ the cross-level interaction between perceived social support (Level-2) socially supportive (Level-1) and negative interpersonal exchanges (Level-1) and drinking contexts was examined. More specifically, I examine whether socially supportive interpersonal exchanges moderate the negative interpersonal exchangedrinking home alone associations for those individuals with stronger perceived support compared to those with weaker perceived support (see Figure 6).

$H_{4}:$ The moderating effects of SIE on the NIE-subsequent solitary drinking at home relationship will be stronger for those with higher perceived social support. 
Daily Social Support and Drinking, 54

\section{Chapter 6}

Methods

\section{Study Overview}

This dissertation examined a secondary data analysis of data collected from a larger study that was directed by Cynthia D. Mohr, Ph.D. (NIAAA Grant R03AA014598). My role was that of project manager whereby I was involved from the outset, from the awarding of the grant through all phases of the data collection. More specifically, I worked with the PI to implement study protocol to achieve study aims. Among my other responsibilities, I was responsible for assisting in the creation and implementation of participant tracking systems, telephone and face-to-face screening, disbursement of participant compensation, correspondence with participants and supervision of undergraduate and graduate research assistants.

In the next section I will describe the participants who were involved in the study and then I will go on to explain the study procedure.

\section{Participants}

Participants included 25 men and 24 women from the Portland community; of those participants 47 participants provided usable data on the handheld electronic interviewer. More specifically, two people were removed from the study because they lost the handheld electronic interviewer. All participants were moderate-toheavy drinkers and over the age of 21 ; but of the remaining 47 participants, the mean age was $36(S D=17.32)$. Further, the majority of the sample was European- 
Daily Social Support and Drinking, 55

American (90\%); $2 \%$ were African-American, $4 \%$ were multiracial and $4 \%$ consider themselves as other. Furthermore, $40 \%$ were married or currently living with a partner, $40 \%$ had never been married, and $20 \%$ were divorced or widowed.

Most participants worked (77\%), mostly working full-time (57\%). In addition, $15 \%$ completed high school, $37 \%$ had some college, and $22 \%$ had a Bachelor's Degree, 13\% had some graduate training, and 13\% completed graduate training. Further, participants reported a wide range of household incomes, with $21 \%$ making less than $\$ 16,000$ per year and $6 \%$ making more than $\$ 132,000$ per year. The median income range was $\$ 27,001$ - $\$ 44,000$ per year and no participants were homeless; in other words, all participants identified a permanent address. After meeting criteria for the current study the eligible participants read and signed an informed consent, which included information on how much how much time the study would take out of their day, as well as how much they would be paid. More specifically, participants were given information about how they could earn the maximum amount of money for participating in this study. For example, participants were paid $\$ 25$ for completing the initial assessment, plus $\$ 1$ for every interview completed. In addition, they were offered weekly bonuses (i.e., \$5) for mailing in the memory chips from the handheld electronic interviewer and completing all the surveys for each week. The total amount that each participant could earn was $\$ 185$. 


\section{Procedure}

Recruitment. Participants were recruited using an Internet bulletin board (i.e., Craigslist), community flyers (e.g., community centers) and local newspapers (i.e., Sellwood Bee) so that the sample would closely represent the adult population in the Portland, Oregon Metropolitan area (see Appendix 1 for sample flyer). Moreover, to further assist with obtaining a diverse sample, ads were placed in Asian and Spanish newspapers. Each method of recruitment asked interested individuals to call our research lab and complete a short 10 minute telephone screening to establish possible eligibility.

Only 7 people decided not to be screened for the study after hearing a brief summary regarding what would be required if they were to be a participant in the study. Various reasons were given by each individual who decided not to participate, including not wanting to carry around a handheld interviewer, blindness, and having to move within the next few weeks. With that said, when individuals called in to be screened and they indicated that they might be having a psychological crisis or severe problems, phone numbers and addresses for local agencies who were equipped to deal with such issues were offered and screening was discontinued.

With a focus on drinking levels, this study enlisted people who did not meet criteria for alcohol abuse or dependence but were drinking more than daily recommended levels (per USDA guidelines, 2000). Specifically, women who were considered moderate-to-heavy drinkers were females who reported that they 
Daily Social Support and Drinking, 57

consumed at least to 2 drinks per day on average, and no less than 6 per week; and men who drank at least to 3 drinks per day on average and no less than 12 per week (see Dufour, 1999). Additionally, potential participants needed to be over the age of 21. The majority of people excluded were ineligible because they were light drinkers or did not drink at all (77\%). Moreover, since the study did not have staff that were trained in diagnosing and assisting individuals who had symptoms of depression, such individuals were excluded, as were any individuals who expressed suicidal thoughts. In the case of depression and suicidality, individuals were offered referral information (i.e., local agency/crisis hotline names and phone numbers.

\section{Initial Assessment.}

Potentially eligible participants were asked to report to the research office located at Portland State University for a face-to-face screening. A trained graduate student used a computerized version of the Computerized Diagnostic Interview Schedule-IV (C-DIS; Robins, Cottler, Buckholz \& Compton, 2000) to further rule out individuals who were alcohol dependent (dependent within their lifetime) or showed signs of current abuse (abuse within the past five years). Eight individuals were excluded after the C-DIS screening; two showed signs of alcohol abuse within the past five years and six met criteria for lifetime dependence. They received compensation for participating in the screening assessment.

In addition, participants completed an initial assessment which was comprised of a battery of psychosocial questionnaires that included individual differences, using an online survey program (i.e., Websurveyor). Completion of the 
Daily Social Support and Drinking, 58 surveys took approximately 45 minutes. Finally, the participants were given an indepth training on how to use the handheld electronic survey. The 20-minute training session included reviewing an instruction manual that provided complete instructions on how to take a survey. A practice survey was then conducted from beginning to end so that all participants were familiar with the handheld electronic interviewer. Furthermore, all participants understood that they were to carry the electronic interviewer with them for 30 days; and three times each day the handheld interviewer would alert the participant to complete the survey.

Daily Interview. Socially supportive exchanges, alcohol consumption and negative interpersonal exchanges were assessed at a random time during each interval (morning, afternoon, and evening) as depicted in Figure 3. In the morning interview (between 10:00-11:30 a.m.), participants were asked to report on support exchanges, numbers of drinks, and negative exchanges from their previous evening's interview until they went to bed. During the afternoon interview (4-5:30 p.m.), they recorded that day's supportive and negative exchanges and drinks from the time they woke up until the time they took the survey. Further, the evening interview (occurring between 8:30-10:00 p.m.) collected support exchanges, drinks, and negative exchanges since the afternoon interview. Time intervals were selected based on prior research conducted in the Portland, Oregon Metro area in which a random digit dial phone survey was conducted by the PI of this study that probed various questions including daily schedules (i.e., "what time do you wake up in the 
Daily Social Support and Drinking, 59

morning"). The average reported time for waking up and going to bed assisted in determining what were appropriate "windows" (e.g., timeframes) for the daily surveys.

\section{Measures}

\section{Initial Assessment}

Perceived Social Support. The 12-item ISEL measure (Cohen \& Hoberman, 1983) assessed the perceived social support (i.e., PSS) functions in the domains of emotional, instrumental, companionship, and validation. The items included statements such as: "If I wanted to go on a trip for a day (for example, to the country or mountains), I would have a hard time finding someone to go with me," and "I feel that there is no one I can share my most private worries and fears with.” Participants were asked to indicate the answer that is most true for them on a 4-point scale, ranging from 1 (definitely false) to 4 (definitely true). The internal consistency coefficient was acceptable, $\alpha=.87$.

\section{Daily Interview}

Socially Supportive Exchanges. Daily socially supportive exchanges were examined using a 7-item modified version of the Positive Interpersonal Exchange measure (Barrera Jr. Sandler, \& Ramsay, 1981; Cutrona, 1986). Participants were asked to appraise how positive each exchange was since the last interview on a 6 point Likert-type scale from 1 (not at all) to 5 (extremely) and 0 indicated that the exchange did not happen (see Appendix 2 for complete list of variables and answer 
Daily Social Support and Drinking, 60

choices). Exchanges that were probed included: "helped me," "spent time /hung out with me," "shared affection/love," "had pleasant conversation," "received compliment," "expressed interest in me," and "other." These items constituted a checklist; therefore internal consistency estimation was not appropriate (see Cronbach, 1951; Spector \& Jex, 1998). To further explain, Spector and Jex (1998) argue that there are key differences between effect and causal indicator measures. Effect indicator measures include items that represent one underlying construct; whereas a causal approach (e.g., checklist) presupposes that a measure consists of discrete components. More specifically, a checklist is comprised of items that are not necessarily interchangeable and some of the items are conceptually distinct. For example, according to the literature, shared affection/love is an indicator of social integration but being helped is an indicator of receiving socially supportive resources; consequently, internal consistency was inappropriate as a measure of reliability (Spector \& Jex, 1998). The items on the supportive interpersonal exchange measure were chosen from the same random phone survey as the previously mentioned (see pg. 53). After examining the appraisals and frequencies of the interpersonal exchanges reported in this survey the most commonly endorsed interpersonal exchange items were chosen to be on the handheld interviewer.

Negative Interpersonal Exchanges. To capture daily stress variability, participants were presented an 8-item modified version of the Negative Interpersonal Exchange measure (Ruehlman \& Karoly, 1991). Participants were asked to appraise 
Daily Social Support and Drinking, 61

how negative each exchange was since the last interview on a 6 point Likert-type scale from 1 (not at all) to 5 (extremely) and 0 indicated that the exchange did not happen (see Appendix 2). Stressful negative interpersonal exchanges that were examined included: "yelled at me," "nagged me," "blocked my goals," "took my feelings lightly," "impatient with me," "ignored me," "hurtful to others" and "other." As previously mentioned, similar to socially supportive exchanges, these items constituted a checklist; therefore internal consistency estimation was not appropriate (see Cronbach, 1951; Spector \& Jex, 1998). As with the social support items, the items on the negative interpersonal exchange measure were chosen from a random phone survey from members of the local community. The most commonly endorsed interpersonal exchange items were chosen to be on the handheld interviewer.

Daily alcohol consumption. Participants were asked to record the number of alcoholic drinks they consumed. In this study, it was not only important to obtain the number of drinks consumed on a daily basis, but to consider the influence that the study may have had on the participants. Specifically, research has shown that participants tend to make personal judgments as to what is socially acceptable by others and perceived social desirability (Swartz, 2007); thus, this can influence reported drinking. For example, if a researcher offers response options that goes up to 50 drinks per interview then the participants may think that, while 50 is a lot of drinks, it is ok to drink that much. In addition, a participant may think that the researcher is looking for them to drink a substantial amount of alcohol if the 
Daily Social Support and Drinking, 62 response options are so high. In other words, the response options can influence the interpretation of the question (e.g., it is ok to drink up to 50 drinks) and the attitudes (e.g., some people must drink up to "50" drinks in a time period) so researchers must be thoughtful (Wittenbrink \& Schwartz, 2007). Taking this in to account, the response options in this study were developed based on previous work that considered these influences on response options when developing their surveys for probing daily drinking behaviors of moderate-to-heavy drinkers (see Mohr et al., 2001, 2005). In addition, previous research has shown that where the responses are placed in the overall survey influences responses. For example, placing them at the beginning results in less accurate responses, the guarantee of confidentiality, and the ease of taking the survey has an influence on the accuracy of responding to surveys (Del Boca \& Darkes, 2003). With that said, participants were first asked how many drinks they had at home and away from home; the response options included 0, 1, 2, $3,4,5,6,7,8,9,10,11,12$ and greater than 12 . If their response was greater than or equal to 1 they were then asked about whether they were alone or with others. The specific question and response options were: While drinking at home were you (click all that apply): 1 . Alone; 2., interacting with others who were drinking; 3. Interacting with others who were not drinking; 4, Not interacting [with others] and others were not drinking; 5. Not interacting and others were drinking.

To ensure accuracy and consistency when reporting drinks, participants received extensive training regarding the size and amount of a standard drink with 
Daily Social Support and Drinking, 63

one drink equaling 1 12-oz. beer, 1 5-oz. wine, and 1 1/2 oz. hard liquor (see Sobel and Sobel; 1992; Dufour, 1999). In addition, each participant was given an alcohol reference guide that included serving size and fluid ounces for most types of drinks.

\section{Data Analyses}

The following sections expound on the data preparation process, as well as variable and covariate creation, variable centering, missing data, trends and serial dependency issues. In addition data structure is discussed, descriptive analyses are offered and hypothesis testing results presented.

\section{Variable Creation}

Drinking variables. I created variables that represented the evening and late night drinking variables using SPSS. More specifically, to create the solitary drinking at home outcome variable, I summed the total number of alcoholic drinks that were consumed at home alone (i.e., no one else present in the home) and home not interacting with others (i.e., others present but were not interacting with them) for two time points (i.e., evening and late-night time points). In this dissertation, the aggregate values across both contexts are considered solitary drinking behavior.

Exchange variables. In SPSS, the socially supportive exchange variable was created by summing all supportive exchanges that were rated between 2 (slightly) to 5 (extremely) for the daytime and evening interviews (the reasons why I am only using these two interviews will be discuss in detail in the next section). Accordingly, higher values indicated a higher number of positively appraised socially supportive 
Daily Social Support and Drinking, 64

exchanges for a given time period. Similarly, all negative exchanges that were rated between 2 (slightly) to 5 (extremely) for each time point were summed to create a summary score for negative interpersonal exchanges for the afternoon and evening interviews. Therefore, higher values indicated a higher number of negatively appraised exchanges for a given time period. I did not use any exchanges that were rated as a 0 or 1 because zero represented a response of "didn't happen" (the participant did not have that experience during that timeframe); and a response of one meant that it was not at all positive (for supportive exchanges) or not at all negative (for negative exchanges).

Between-person variable. The between-person variable, perceived social support, was created by summing the individual scores on the scale to create a composite score (per Cohen \& Wills, 1985). Thus, the results would reflect the differences of average levels of social support between individuals on perceived support.

Lagged variables. Since this dissertation examined temporal ordering of the variables of interest, lagged variables were created. In other words, I assessed how experiences (social support and negative exchanges) related to later drinking; thus, lagged variables were created. To do that I created syntax in SPSS that computed a lagged variable for supportive and negative exchanges. By doing this, I shifted the data for the interpersonal exchanges down one cell in SPSS; thus, reducing the total number of data points per person by one. 
Daily Social Support and Drinking, 65

Centering. Before continuing on and explaining how the interaction terms were created it is important to first explain how each variable was centered; thus, centering is a central component in understanding the interaction terms. Enders and Tofighi (2007) point out that in multilevel models centering allows for the creation of a meaningful zero point. Since I was interested in examining average withinperson change, each Level-1 predictor was person-centered so that an average within-person score can be obtained (Enders \& Tofighi, 2007). In other words, I was able to examine the unit change in a person's score based on their average rather than zero. This was done by taking each person's (within-person) mean and subtracting it from their raw within-person score in SPSS. This allowed for a more meaningful interpretation (Enders \& Tofighi, 2007). It is also important to note that by centering, the values changed, but not the scale (Enders \& Tofighi, 2007; Hoffman \& Gavin, 1998; Kreft, de Leeuw, \& Aiken, 1995); for example, I was able to examine their drinking on days when social support is average (their mean) with centered variables rather than examine their drinking at times when social support is zero. Moreover, when $\mathrm{X}$ is centered, the regression coefficient does not change and the regression coefficient can be interpreted as the effect on the within-person mean of $\mathrm{Y}$ for each one unit increase or decrease in X (Enders \& Tofighi, 2007; Hoffman \& Gavin, 1998; Kreft, de Leeuw, \& Aiken, 1995). Thus, when daily supportive exchanges are centered the regression coefficient can be interpreted as drinking levels on days with an average number of daily supportive exchanges; and as people report one more 
Daily Social Support and Drinking, 66

(i.e., one standard deviation) daily supportive exchange the drinking level goes down by $\mathrm{X}$.

The Level-2 variable, Perceived Social Support (PSS), was grand mean centered. By grand mean centering the Level-2 variable the intercept represented the expected value of the outcome variable at the mean value of my Level-2 predictor (Hoffman, 1998; Hox, 2002). That is, I was able to assess the average betweenperson levels of perceived support. As with the Level-1variables, this was done for ease of interpretation. By centering the Level-1 and Level-2 variables the likelihood of multicollinearity was reduced. Furthermore, Bryk and Raudenbush (1992) suggest that centering will make model estimations easier when examining main effects and interactions in the same model.

Interaction Term. My estimation of interaction effects for Hypothesis 2 and Hypothesis 4 require addition data preparation. Consequently, I needed to create my moderating variables. Following instructions from Aiken and West (1991), I used the standardized values for each variable (i.e., Z-scores), for ease of interpretation and so that I could solve the equation in order to graph the interaction effects (Aiken \& West, 1991). More specifically, once my Level-1 variables were centered I obtained a Z-score for each variable (Aiken \& West, 1991). This allows me to have an idea as to how many standard deviations from the mean my estimate is. For the interaction term, I then multiplied the Z-scores together to obtain the moderating variable. By transforming the variables, a one-unit difference now represents a one-standard 
Daily Social Support and Drinking, 67

deviation difference. It is important to point out that while transforming these variables does not change the relationship among the values of each variable, I am not taking in to account the variance of each person's variance. In other words, rather than having a number of different standard deviations across individuals, I now have a standard deviation of 1 and -1 for each person. While this is important for solving the equations and graphing, I am losing information by using this technique.

Since adding interaction terms adds instability to models (i.e., multicollinearity), Hox (2002) and Snijders and Bosker (1999) argue that when examining interaction effects it is essential to test the main effects first, without the interaction term included in the model. In addition, Hox argued that the (main-effect) variables that are used to create an interaction term have very different meanings depending on the whether the model includes the interaction effect. More specifically, Hox (2002) argued that if a model has an interaction term the regression coefficient of one of the main effect variables is the "expected value of the regression slope for the case that the other variable is equal to zero, and vice versa" (pp. 58). This is especially problematic when variables have been centered and zero has no real meaning for the variables being used. For that reason, the main effects were reported in tables for models that included interaction terms but they were not interpreted. For this dissertation, I wanted a clear understanding of the impact of my predictors on my outcome variable without the added complexity of an interaction term; thus, I ran each model with the main effects only first, then I added the 
interaction terms in the subsequent models.

In addition, even if the predictors do not have significant slopes the interaction term can still be tested (Snijders \& Bosker, 1999). Snijders and Bosker (1999) suggest that it is best to model within-person interaction terms as fixed because they are "often hard to interpret" or they lead to incorrect interpretations. Moreover, Snijders and Bosker (1999) have demonstrated that random within-person interaction terms have been shown to cause lengthy iteration processes of the estimation algorithm, which results in the algorithm failing to converge (pp. $92 \&$ 95). In other words, not fixing the within-person interaction terms would likely result in HLM not being able to produce (accurate) results. With that in mind, the within-person interactions were modeled as fixed effects and were interpreted as the moderating effect of socially supportive exchanges on the negative exchangedrinking relationship.

\section{Missing data}

As Tabachnik and Fidel argue (2001), it is key to have a clear understanding the pattern of missing data rather than focus on the amount of missing data. With that said, in this section, I will examine the types and the amounts of missing data because missing data

Little and Rubin (1987) argued that data that are missing can be missing at random (MAR), missing completely at random (MCAR) and non-ignorable missing (NIM). In MCAR data, missingness is completely independent from all other 
Daily Social Support and Drinking, 69

variables. MAR, which is typical in multilevel models (Hox, 2002) assumes that information regarding the missing data are recognized and can be examined (Little \& Rubin, 1987). Moreover, MCAR is a more restrictive assumption than MAR since it assumes that missingness may well depend on additional observed variables in the models (Hox, 2002; Little \& Rubin, 1987). NIM are missing data that are systematic in nature; thus, they must be modeled to obtain accurate parameter estimates (Allison, 2002; Little \& Rubin, 1987). Allison (2002) argues that to obtain accurate estimations the researcher must have an exceptionally clear understanding about the NIM because the choice of model that is used can have a significant effect on the results.

In daily process methods, missing data can pose a significant problem; specifically, attrition can result in substantial missing data. Hox (2002) argued that in multilevel models data are not generally MCAR because some individuals may be more likely to drop out or not answer surveys, than others; thus, data are usually MAR (Hox, 2002). It is important to find the sources of incomplete data, if possible. Hofer and Hoffman (2007) put forth that there can be a number of reasons for this missing data such as unit nonresponse (i.e., attrition from the study, mortality). As I went through the data I found that in this study, we had two individuals (1 man, 1 woman) who provided data that was unusable because of corrupt data that could not be retrieved or restored; thus, the resulting sample was comprised of 47 individuals. We determined that this was due to a malfunction of the handheld surveyor. Hofer 
Daily Social Support and Drinking, 70

and Hoffman (2007) categorize this as attrition as a nuisance and they argue that it is appropriate for researchers to go forward with analyses with the best (and most) available data. Hofer and Hoffman (2007) also state that researchers need to be aware of attrition that may be part of a natural process. For example, in my study missing data or attrition may be due a person who is drinking at very high levels and does not want that information to be revealed. Their suggested solution is to be aware that this is a possibility when designing the study and include possible covariates (i.e., individual and contextual measurements) that are apt to be related to the likelihood of missing.

To address this possible missing data issue, I examined the dataset to determine how many people stopped taking the surveys early. We had two participants who were removed from the study because they lost the equipment and two participants who only answered approximately two-thirds of all the questions. I then proceeded to conduct an analysis of variance to examine whether those individuals who left the study early drank significantly more than those who remained. In addition, I examined whether they reported more daily socially supportive and negative exchanges than those who remained in the study. Results revealed that there were no significant differences among any study variables of interest between individuals who left the study early $(n=4)$ and those who remained the entire duration of the study $(n=43)$. Specifically, there were no difference in mean drinks per drinking occasion $[F(1,37)=1.37, p=.25]$, mean supportive 
Daily Social Support and Drinking, 71

exchanges $[F(1,45)=3.23, p=.08]$, negative exchanges $[F(1,45)=.70, p=.41]$ or perceived support $[F(1,41)=1.47, p=.23]$. In sum, there did not appear to be cause for concern about study attrition.

Finally, in the following subsections I addressed item nonresponse attrition; more specifically, I examined information about compliance rates and a details about missing data such as how many people completed less than $30 \%$ of the surveys and how many individuals completed $70 \%$ or more of the surveys. Moreover, I offered an explanation as to why it was not possible for participants skip questions, which otherwise would have resulted in more missing data.

Compliance. To assess the amount of missing data from the entire study (all 3 daily interviews), I first examined the general compliance of the participants on the variables of interest. Specifically, I assessed how many surveys each person completed and added them together to obtain an overall total number of completed surveys over the course of the study (3510). I then divided the completed participant surveys from the total number of surveys I should have had if each person completed every survey (47 individuals x 90 surveys $=4230 ; 3510 / 4230)$. At that point I was able to establish a compliance rate of $83 \%$ which is analogous to the compliance rate reported in similar published daily process studies (see Hufford, Shields, Shiffman, Paty \& Balbanis, 2002; Mohr et al., 2005).

While understanding the relationship between missing data and key variables is important, Raudenbush and Bryk (2002) state that as long as each participant has 
Daily Social Support and Drinking, 72

at least one observation, results can be analyzed (see page 199 in Raudenbush \& Bryk, 2002). More specifically, using a statistical program such as HLM v6.0 allows the missing data to be analyzed and weighted. In other words, cases that included fewer observations can be (and were in this study) weighted less than those who provided more observations in HLM. This does not mean that having a clear understanding of the association between missing data and predictors and outcomes is not important. In fact, missing data can have serious implications for validity. For example, internal validity can be threatened if participants change their behavior because they are in the study, otherwise known as reactivity. This issue will be examined in the section that addresses trends in the data (see page 79), but it is important to point out that previous research has determined that by not having participants able to review their answers to questions in a survey and by having them answer questions about many different kinds of behaviors and feeling, reactivity can be reduced (Affleck et al., 1999; Marco, Neale, Schwartz, Shiffman, \& Stone, 1999). In this study, those precautions were taken. While overall compliance was high there were concerns with the present data that included participants skipping questions and missing data for each variable of interest; each of which will be discussed next. Answering questions on the surveyor. The handheld electronic interviewer used in this study required participants to answer each question before it allowed participants to go on to the next screen; thus, eliminating the problem of people skipping particular questions. More specifically, this eliminated surveys in which 
Daily Social Support and Drinking, 73

people were able to pick and choose what questions they wanted to answer unless there was a technical glitch in the program or someone discontinued the survey altogether. However, I wanted to examine the missing data associated with each variable. Consequently, in the next sections I will examine variable-specific missing data.

Missing data for each variable. If each person answered the survey every time the total number of possible surveys is 2820 . More specifically, 47 individuals could have answered 2 interviews per day over the course of the 30 day study; thus, the total number of possible data points for this variable was 2820 . I ran a frequency of all the missing data points for drinking in the evening and late evening. Results indicated that there were 2203 valid data points with 617 missing data points for the drinking variable. Similarly, there were 2221 valid data points positive and negative interpersonal exchange and there were 598 missing data points for negative and supportive exchanges.

There are two important points to be made regarding the differences in missing data between my predictor variables and my outcome variable. First, the predictors were lagged and when a lagged variable is created in SPSS it shifts the data in the column down one line for each person; thus, resulting in a missing data point for each person's first interview. Since each person started the study at different interview times the number of missing data points for say, interpersonal exchanges, was not the same for everyone because of the lagging. The total number 
Daily Social Support and Drinking, 74

of missing data points due to lagging was 26. Secondly, excluding the lagged missing data points, there were still more missing data points for drinking than daily exchanges. However, this discrepancy can almost be entirely accounted for by the data coding decision to remove the drinking observations that involved multiple drinking at home contexts (please see the measures section). This examination suggested that these missing data points were not NIM missing data or systematic biases in participants' responding.

Survey completion rates. In this section, I examined the completion rates of the between- and within-person surveys. The changes are as follows: I probed what percentage of people responded to the between-person variable, perceived social support. Next, I examined the daily diary completion rates; what percentage of people completed most of the surveys and how many people completed only a few surveys. While researchers have not determined an acceptable (or required) level of survey completion rates (Babbie, 2007; Salant \& Dillman, 1994) a leading expert in the field, Don Dillman (2007) has suggested that, in general, using $70 \%$ as a cut-off for high completion rates is acceptable. Moreover, he has also suggested that it is difficult to determine what "low" completion rates should be, but in general, $30 \%$ will give researchers a sense of low completion rates. With that in mind, I examined individuals who had a relatively high completion rates; specifically, those who completed at least $70 \%$ of their daily surveys and the questions for the between- 
Daily Social Support and Drinking, 75

person measures. In addition, I examine those who completed only $30 \%$ of the daily surveys and the question for the between-person measures.

The between-person survey was only conducted once (during the initial assessment phase of the study). First, I inspected the completion rates of the between-person variable of perceived social support. Results indicated that $96 \%$ of the individuals completed at least $70 \%$ of the questions for this measure (see Table 1), as well as the other between-person psychosocial measures. Consequently, only $4 \%$ completed less than $30 \%$ of the questionnaires. One hundred percent of the participants reported gender and marital status information.

Next, I examined the survey completion rates for the within-person variables of supportive and negative exchanges and the drinking variable (see Table 2). For supportive and negative exchanges, $70 \%$ of the participants completed $70 \%$ of all the surveys; in fact of those who answered $70 \%$ or more of the surveys, almost one quarter of those participants (24\%) missed only 0-1 surveys over the course of the study.

For the drinking variable, most people $(81 \%)$ completed $70 \%$ of all the surveys. Similar to the exchange variables, of those participants who answered $70 \%$ or more of the surveys, almost of quarter of those individuals (24\%) missed only 0-1 surveys over the course of the entire study. Finally, for the drinking variable only one person completed less than $30 \%$ of all the surveys (see Table 2). 
Daily Social Support and Drinking, 76

In sum, examination of the missing data determined that, in general, the missing data were MAR. Due to the programming of the handheld electronic interviewer (i.e., not allowing people to skip questions) and after my review of the missing data, I do not believe that I have serious missing data issues. Moreover, since I am using a statistical program (i.e., HLM) that is robust to missing data issues and, more specifically unbalanced data, I did not delete participants due to missing data; however, I did recognize this point in the limitations section of my discussion.

\section{Distribution of measures and tests of outliers.}

With the variables now created and the missing data assessed, I next examined the distributions of variables by reviewing frequencies histograms and descriptive statistics of all variables of interest (Mertler \& Vanatta, 2005; Howell, 2002). In general, it not necessary for predictors to have normal distributions, but to satisfy the assumption of normality, it is important for outcome variables to be normally distributed when using traditional statistical programs (Mertler \& Vannata, 2005). In multilevel models this assumption is often violated; but statistical programs such HLM 6.0 are equipped to handle non-normal distributions. With that said, before I began the analyses I still wanted to familiarize myself with the data so I examined the distribution of the outcome variable as well as the predictors. Examining the predictors can provide critical descriptive information, such as how many exchanges were people experiencing at each time point and what were the daily average number of exchanges reported. Also, examining the predictors is 
Daily Social Support and Drinking, 77

necessary to make sure that there were no errors in the data cleaning process. In the next section I will examine the distribution and possible outliers of the outcome variables, which includes a discussion of the issue of structural zeros.

Distribution and outliers of the outcome. Before I examined the distribution of the outcome variable, it became apparent that this dataset was particularly complex. Specifically, when I began investigating the frequencies of the daytime and evening drinking behaviors I found that most people did not drink during the daytime hours. This was likely due to the fact that $77 \%$ were employed and they likely did not have the opportunity to report daytime drinking at home because they were working; thus, the dataset contained structural zeros. In contingency tables, a cell that contains a structural zero is a cell in which the expected value is zero due to the fact that it was very unlikely or impossible that any observations would fall into that cell. In other words, it was unlikely for them to report drinking in the day; therefore, they did not contribute to the likelihood function or model fitting (Berger \& Zhang, 2005). In this dataset most people worked (77\%); thus, it was not anticipated that many people in our sample had an opportunity to drink during the workday (i.e., middle of the day on weekdays). Moreover, empirical evidence has indicated that in samples of moderate-to-heavy drinkers the vast majority of the drinking takes place in the evening hours (see Swendsen et al., 2000). Taking this into account, I decided not use the second daily interview (which comprises daytime drinks) to assess solitary drinking levels; however, I did use this timeframe to assess interpersonal 
Daily Social Support and Drinking, 78

exchanges. More specifically, I removed the daytime drinking data which assessed drinking from the time a person woke up until the late afternoon.

After removing the second interview drinking data from the dataset, frequencies and histograms were computed using the solitary drinking variable so that I could visually examine the distribution. This provides helpful information in that I can better determine the range in which people drank and how many people are clustered around the top and the bottom of that range. It was apparent that the majority of individuals' drinking responses were clustered around the lower end of the scale (i.e., 1877 data points were zero and 233 data points were 1), with only 4 data points with frequency values of between $6-13$. Since participants in this study were considered moderate-to-heavy drinkers it was not surprising that the outcome variable, solitary drinking at home, was negatively skewed (skewness=6.34); with $44 \%$ of data points for the solitary drinking variable comprising zeroes. Most participants' responses were between 0-1 (1877 data points were zero and 233 data points were 1) compared to only 4 data points with frequency values of between 6 13. Thus, I uncovered evidence of heteroscedasticity and zero-inflation (Howell, 2002; Miller \& Miller, 2008). Miller and Miller (2008) argue that to analyze data such as these as if they were normally distributed would result in inflated standard errors, erroneous and inconsistent parameter estimates and biased findings.

Furthermore, zero-inflated, non-linear skewed data transformation can cause additional problems. More specifically, a transformation to attain variances that are 
Daily Social Support and Drinking, 79

relatively equal in zero-inflated data can result in non-normal residuals (Bryk \& Raudenbush, 1996; Miller \& Miller, 2008). Bryk and Raudenbush (1996) suggest that there are times when it is not reasonable for data to be linear and normally distributed and no transformation could (or should) be used (pp. 291). With that in mind, rather than conduct a transformation in an attempt to normalize the distribution it seemed logical to use a more adequate model. Previous research suggests that for data that includes non-linear, skewed count data, Poisson models are the most appropriate models to use (Armeli et al., 2005; Bryk \& Raudenbush, 1996; Miller \& Miller, 2008). Accordingly, I deemed that no transformations were necessary with my outcome variable and Poisson Models in HLM was appropriate to use (Raudenbush, et al., 2000).

Furthermore, when I examined the histogram closely it revealed that I had one data point that was not clustered with the rest of the distribution; more specifically, for one person the largest amount consumed at one interview at home alone was thirteen. For the rest of the participants, levels of reported drinking ranged anywhere from 0 and 6 drinks at home alone. To further investigate this potential issue, I aggregated the sum of all drinks per person. The frequency and histogram revealed that one person drank more at home alone over the course of the study ( $n=82$ drinks) than anyone else; the total drinks at home alone for the other participants ranged between 0-42. Further analyses revealed that this person drank on average 3 drinks per interview with 2 interviews being higher (6 drinks). In 
Daily Social Support and Drinking, 80

addition, $72 \%$ of the time that this person consumed alcohol, they consumed 3 drinks or less. Since my data were person-centered and the within person analyses were comparing each person against their own average (not that of the group), it did not seem likely that this individual's data would heavily sway the overall estimates. However, to be cautious, I determined whether removing this person's data would drastically change the overall mean, median, and standard deviation for the drinking variable. So I aggregated the within-person drinking levels (across study days) and created two datasets: One that had this potentially problematic person's data and one that had his/her data removed. Central tendency and variability values obtained including the possible outlier were: Mean $=.23$, Median $=.09$ and $S D=.44$. Whereas, values excluding the outliner were: Mean $=.17$, Median $=.09$, and $S D=$ .21. Further, t-test analyses indicated that there were no significant differences between the mean value of drinking home alone including and excluding the potentially outlying data $[t(46)=.87, p=.39]$. In sum, I was able to conclude that this one individual's higher level of solitary drinking at home was not problematic in terms of potentially influencing my hypothesis testing.

I conducted one final set of analyses to determine whether there were any outliers that I could not detect visually using Mahalanobis Distances. Mahalanobis Distances identify the distance of cases from the centroid of the other cases (centroid is the mean of all the other variables; Tabachnick \& Fidell, 1996). Specifically, I conducted a linear regression to obtain Mahalanobis Distance values for the solitary 
Daily Social Support and Drinking, 81

drinking variable; then a chi-square test was conducted to obtain a critical value. A critical value of 99.61 was obtained for a chi-square with 64 degrees of freedom and with $p=.001$. Subsequently, there were no values greater than the critical value, thus determining that there were no outliers in the outcome variable.

In sum, due to people working in the day and not having opportunities to drink in the day (i.e., structural zeros), daytime drinking data was excluded from the analyses. In addition, based on preliminary analyses I concluded that there were no outliers or problematic data issues. In addition, due the outcome variable being a count variable with significant number of responses that were zeros (i.e., zeroinflated), Poisson models will be used in all subsequent multilevel models. Distribution and outliers of predictor variables. Examination of the predictors included a study of the range of responses, accuracy of the data (e.g., errors in the cleaning and coding process) and whether the data collected from my sample is comparative to other studies who have examined moderate-to-heavy drinkers. Additionally, preliminarily information can provide vital explanatory information, such as the number of positive and negative exchanges at each time point and the average number of exchanges reported across days. For those reasons, I conducted a thorough visual inspection of the data; then I ran frequencies, histograms, and Mahalanobis Distances.

Frequencies and histograms revealed that the negative exchanges variable was skewed; this was due to a large number of zeros. As shown in Table 3 there 
Daily Social Support and Drinking, 82

were 1967 occasions (across the two time points) in which people reported 0;

meaning that there were a substantial number of times people did not experience a negative exchange. The skewness value was 3.27; yet after the variables were person-centered they were far less skewed (1.84). The negative interpersonal exchange measure is modified version of the TENSE (Ruehlman \& Karoly, 1991), adapted for purposes of the present study based on focus group feedback and then validated in a random phone survey. Thus, I do not have reason to believe that the negative exchange measure was not valid although there was a substantial number of zeros. In addition, we offered people the choice of "other" and over the course of the study people only used that answer option $4 \%$ of the time in the timeframes that I was probing. This suggested that the participants were not experiencing a lot of negative exchanges other than what we listed; our participants were only reporting negative exchanges $30 \%$ of the time. Previous work in this area has found similar results. Using a sample of older individuals (aged > 70), Rook (2001) found in her daily diary study that on average these individuals only reported .34 per day. Similarly, Mohr et al. (2005) found that their participants reported an average of .30 negative exchanges in the morning over the course of their study and an average of .47 negative exchanges for the afternoon. My participants reported an average of .65 negative exchanges per time point across the study days.

In contrast to negative exchanges, the normality of the distribution of daily socially supportive exchanges was apparent in the histograms and frequencies. When 
Daily Social Support and Drinking, 83

not centered the daily socially supportive exchanges were not skewed (skewness $=$ .69) and when it was person-centered that did not change (skewness=.34). Given concerned that the supportive exchange measure was not capturing all daily supportive exchanges, I probed the supportive exchange option of "other" just as I did for negative exchanges. I found that over the course of the study people only responded to the "other" option $6 \%$ times (out of a possible 4230 times). This, in conjunction with the number of times people reported not experiencing daily negative exchanges, suggests that this is a relatively low stressed, supported group of individuals. Moreover, in a daily diary study examining positive and negative exchanges, Rook (2001) found that the participants reported almost 6 times more positive exchanges on a daily basis (total of 6.52) compared to negative exchange (total of .34). In sum, it is argued that supportive/positive exchanges are by far more common than negative exchange (Rook, 2001), pattern which is affirmed in my study.

The frequencies and histogram for my between-person measure, perceived support, had a normal distribution. Moreover, there were no data points that were more than two standard deviations from the mean. The skewness value was .25 , supporting this notion; thus, I concluded that there were no problems with this variable.

Lastly, I examined Mahalanobis Distances for my supportive and negative interpersonal exchange variables and the between-person perceived support variable. 
Daily Social Support and Drinking, 84

First, Mahalanobis Distance values were obtained for daily supportive interpersonal exchanges. Next, a critical value of 10.38 was obtained for a chi-square with 1 degrees of freedom and with a significance level of $p=.001$, as recommended by Tabachnick and Fidell (2001). No data points were over the critical value. Similarly, there were no values over the critical value for the between-person social support measure (perceived social support); thus, there were no outliers in the present sample.

Next, Mahalanobis Distance values were obtained using linear regression in SPSS for the negative interpersonal exchanges, and then a chi-square test was conducted. Subsequently, a critical value of 10.82 was obtained for a chi-square with 1 degrees of freedom (degrees of freedom are determined by the number of variables being examined) and with $p=.001$. Next, the highest critical values were examined for each participant. Seven values were greater than the critical value of 10.83 , with the highest critical value for negative interpersonal exchanges having a value of 49.28. For interpretation the negative exchanges were person-centered; consequently, when that step was conducted the issue of outliers was eliminated. No values were over the critical value.

\section{Data structure}

Since daily process data such as these contains both within-and betweenperson observations it is considered to be hierarchically structured data. In addition, these data are considered to be unbalanced because both observed and missing data 
Daily Social Support and Drinking, 85

were included in this data set and since individuals had different numbers of missing data points. To address this potential problem I used Hierarchical Linear Modeling (HLM, v6.0; Raudenbush et al., 2000), because it was developed to handle unbalanced data of this sort. HLM was an appropriate program because it is capable of generating slopes and intercepts for unbalanced data. By using HLM, I was able to specify two regression equations; a within-person regression model (Level 1) and a between-person regression model (Level 2). The within-person equation included a within-person outcome (e.g., solitary drinking at home) that was modeled as a function of a Level 1 (within-person) predictor such as social supportive exchanges. The between-person equation (Level 2) modeled the intercepts and slopes from Level 1 as a function of the between-person predictor (i.e., perceived social support).

HLM offers two types of results, one for the unit-specific models and one for the population-average model. Raudenbush et al. (2001) explain that the models offer very similar results unless nonlinear models are used. In those cases, the population-average effects represent a sample average and are considered to be a more appropriate method of estimation (Neuhaus, Kalbfleisch, and Hauck; 1991; Newson, 2009); thus, those are the results in this dissertation.

Trends and Serial Dependency.

With this type of data it is important to consider serial dependency. Serial dependency is when the data points closest in time are more similar than those that are further apart in time (Cook, \& Campbell, 1979; Judd \& Kenny, 1981). 
Daily Social Support and Drinking, 86

Researchers have calculated autocorrelations as a way of measuring serial dependence. It is important to note that an autocorrelation is not exactly a Pearson product-moment correlation and that specific statistical programs are needed to compute it (Judd \& Kenny, 1981, p. 139). With that said, when examining temporally ordered data, autocorrelations (e.g., serial dependencies) can be a significant issue (see West \& Hepworth, 1991) especially with day-of-week trends typically reported in the alcohol consumption literature (e.g., Armeli et al., 2000). More specifically, temporally ordered data of this sort are susceptible to trends such as weekday and weekend trends and fatigue over the course of the study (less solitary drinking at home reported). Consequently, it was important to look for trends in these data.

Day-of-week contrasts. An example of day-of-week effects would be participants reporting more drinking on Saturday than other days of the week. To examine such a possibility, six dummy variables were created as day of week orthogonal contrasts and they were modeled as fixed effects (see Bryk \& Raudenbush, 1992, p. 151). Drinking on Sunday through Saturday was compared against drinking on Tuesday (the day on which people drink the least; see Argeriou, 1975), which was held constant (Carney, Armeli, Tennen, Affleck, \& O’Neil, 2000; Mohr et al, 2001). I regressed drinking on the day-of-week covariates by running a Poisson (constant exposure) model while adjusting for overdispersion. To clarify, overdispersion is when there is more variability in a data set than is generally 
Daily Social Support and Drinking, 87

expected in an uncomplicated statistical model and is common in Poisson models; thus overdispersion was accounted for in all models (Berk \& MacDonald, 2007). Results of the final estimation of fixed effects (population-average model with robust standard errors) revealed that only one day of week was significantly and inversely related to solitary drinking and that was Thursday, $[t(2170)=-2.96, p=.004]$. Because of this result, day of week was modeled in all analyses.

Trends over the course of the study. In addition, in multilevel data such as these there may be trends over the course of the entire study. As mentioned in the missing data section of this dissertation, reactivity can be an issue in studies such as this one, as well as compliance. I have already addressed compliance in the missing data section; thus, I will now turn to reactivity issues. More specifically, it is possible that individuals who are asked to record daily drinking behaviors may drink more or less over the course of the study (see Affleck et al., 1999). To examine such possibilities, I regressed each predictor and the outcome variable on study day (West \& Hepworth, 1991) while controlling for time of day. Results indicated that time in the study did not significantly relate to drinking levels $[t(2117)=-1.11, p=.27]$. Time in the study also did not significantly increase or decrease the strength of the daily supportive exchanges, $[t(2117)=-.69, p=.50]$. However, there was a significant inverse association between study day and negative exchanges, $[t(2117)=$ $-7.23, p<.001]$. This may be due to people reflecting on their stressors (i.e., reactivity; an issue I will return to more fully in the discussion; Affleck, 1999; Hayes 
Daily Social Support and Drinking, 88

\& Cavior, 1980). Researchers have examined this very issue - does monitoring one's own behavior, in fact, change a person's behavior (Affleck, et al., 1999). It is possible that recording negative exchanges each day, it might alter how individuals appraise such events. Research has shown that monitoring behaviors and feeling can be an effective therapeutic device (Tennen et al., 2000) but inspection of temporal trends in studies similar to mine has not shown this to be a significant issue (Affleck et al., 1999). It could be similar to the effects from daily expressive writing interventions in which working through daily reports on stressors can change people's emotional and cognitive experience of the stressor (Pennebaker \& Chung, 2007). Recall that only exchanges that were appraised as slightly-to-very negative were included in my exchange variable score; benign negative exchanges were not included. As such, people may have altered their appraisal of negative but not positive exchanges over time. Yet, according to Judd and Kenny (1981), although it is vital to account for trends in outcome variables, it is not critical to account for trends in every variable. As a predictor variable, I did not need to model this trend in my analyses.

Time-of-day covariate. Furthermore, in this study multiple interviews were nested within day (i.e., 3 interviews per day). Current research has suggested that the time of day influences drinking behavior (see Kranzler, Armeli, Feinn, \& Tennen, 2004; Mohr, et al., 2005; Park et al., 2004); thus, a time of day covariate was added to the model to control for this issue. More specifically, one dummy code was 
Daily Social Support and Drinking, 89

created to differentiate morning (which probes late night drinking) and afternoon intervals. Similar to the day-of-week covariates, the time-of-day covariate were modeled as fixed effects (see Bryk \& Raudenbush, 1992). Analyses indicated that there was not significant association between the time of day covariate and solitary drinks at home, $[t(4003)=1.83, \mathrm{p}=.07]$. However, because it represents a nesting in the structure of the data, I continued to include time of day as a covariate in all models.

Autoregressive error analyses. As previously mentioned, temporally ordered data such as the data used in this dissertation, often contain cyclical components and serial dependencies which can a pose a problem to analyses by biasing parameter estimates and distorting tests of significance (West \& Hepworth, 1991). Previous work (see Mohr et al., 2001; Mohr et al., 2005) addressed this problem by adding covariates. To be sure that the day-of-week and time-of-day covariates in this study addressed possible autoregressive error I initially planned on adding six dummy variables to control for day-of-week variation and I proposed to model the variance of the Level-1 variables as autoregressive in HLM. Unfortunately, HLM was unable to perform this operation on these type of data. More specifically, it required data with indicator variables for each time point. That would necessitate 30 dummy variables for the thirty days in which people were in the study; that was not feasible because it would dramatically reduce the degrees of freedom and consequently reduce power dramatically. 
Daily Social Support and Drinking, 90

To resolve this issue I used SAS PROC MIXED (Littell, Milliken, Stroup \& Wolfinger, 1996). This sophisticated program has been used in previous research with models very similar to the models being used in this dissertation (see Mohr, Armeli, Tennen, Carney, Affleck, \& Hromi, 2001); thus, I decided to analyze the time varying models in this program.

Interestingly, I found that the AR(1) error structure was statistically significant when I ran the empty model; yet once the day-of-week covariates and predictors were added to the model, the $\mathrm{AR}(1)$ error was no longer significant. These results suggest that it is important that I keep the day-of-week covariates to control for the $\mathrm{AR}(1)$ error. In conclusion, the autoregressive error that was present in the empty model was no longer an issue once the predictors were added to the model; therefore, it was not necessary to model the AR(1) error. Thus, I used HLM in all hypothesis analyses. 
Daily Social Support and Drinking, 91

\section{CHAPTER 7}

Results

\section{Descriptive Information}

To obtain a sense of how many exchanges were happening during the day compared to evening, I first examined the frequencies of the exchange variables. Results indicated that over the course of the study, there were 1034 times during daytime interviews in which people reported having supportive exchanges. In addition, 977 times people reported supportive exchanges in the evening (see Table 3). Consistent with previous research (see Bolger et al., 1988), people in my study reported far fewer negative than supportive or positively appraised exchanges. Specifically, there were 446 reports of daytime negative exchanges (see Table 3). Similarly, there were 314 times people reported having negative exchanges during the evening (less than half as many as supportive exchanges).

Next I examined average drinks per drinking occasion (see Table 4). Results revealed that when people consumed alcohol they drank, on average, $1.42(S D=.78)$ solitary drinks at home. Furthermore, the number of average drinks per drinking occasion ranged from 1 to 4.6 drinks. Interestingly, average number of drinks did not correlate with supportive or negative exchanges; nor did they correlate with gender or perceived support (see Table 4).

Furthermore, means, standard deviations and correlations are presented in Table 4. Results indicated that people, on average, reported $2.24(S D=1.12)$ daily 
Daily Social Support and Drinking, 92

supportive exchanges and an average of .65 $(S D=.88)$ daily negative exchanges.

Interestingly, average supportive exchanges and average negative exchanges were significantly correlated $(r=.53, p<.01)$. This suggests that individuals who reported more negative exchanges during the study also reported more supportive exchanges. Furthermore, average supportive and negative exchanges were not significantly correlated with average number of drinks. Considering the focus of this study, I found it interesting that an increase or decrease in the number of exchanges (support or negative) was not correlated with drinks. Another interesting point is that gender was not correlated with any predictor or the outcome variables. While some studies have found significant gender differences in drinking outcomes the findings have not been consistent across samples and studies (see Mohr et al. 2005). Thus, gender was not included in the following analyses.

\section{Possible Covariates}

Residential Status. Preliminary analyses examined whether residential status was significantly related to the number of solitary drinks at home. Hierarchical linear modeling was conducted whereby the number of adults and children who lived in the home were regressed on number of solitary drinks at home while controlling for dayof-week and time-of-day. Results indicated that living with adults did not significant predict the number of drinks at home alone $(b=.02, p=.19)$. Similarly, the number of children living in the home also did not significantly predict solitary drinking at home $(b=.01, p=.23)$. 
Daily Social Support and Drinking, 93

Marital Status. Preliminary analyses also examined whether there are significant differences in solitary number of drinks at home among individuals who are married or in relationships compared to those who are not. Based on previous research linking loss of marital status (i.e. widowed, divorced) and increased drinking (see Wilsnack \& Cheloha, 1987), I wanted to consider the possible association between marital status and drinking. Accordingly, I categorized people by marital status (i.e., single/never married=1; married or in a cohabiting Relationship $=2$; and whether they were divorced or widowed=3). Hierarchical linear modeling was conducted whereby marital status was regressed on solitary number of drinks at home while controlling for day-of-week and time-of-day. Results indicated that marital status did not significant predict solitary drinking at home, $(\mathrm{b}=.01, p$ $=.49)$.

In sum, marital status, number of adults and number of children living at home, did not significantly predict solitary drinking at home. Thus, no covariates were added to the subsequent models.

\section{Statistical Models}

The daily diary data consisted of hierarchically structured data in which 60 data points are nested within each individual (2 times per day for 30 days; e.g., Affleck, Zautra, Tennen, \& Armeli, 1999; Bryk \& Raudenbush, 1992; Kenny, Bolger, \& Kashy, 2000; Snijders \& Bosker, 1999). Multilevel modeling allows for between- (i.e., level-2) and within-person (level-1) parameters to be estimated. This 
Daily Social Support and Drinking, 94

method is ideal because traditional OLS (ordinary least squares) may produce inaccurate parameter estimates (Cohen, Cohen, West \& Aiken, 2003; Hox, 2002). Moreover, in nested data the assumption of independence is violated, which can be problematic in OLS, because standard error estimates are too small, which result in spurious results (Hox, 2002; Snijders \& Bosker, 1999). Accordingly, to examine the hypotheses in this dissertation, multilevel techniques were used.

HLM 6.0 (Raudenbush, et al., 2005) provides coefficients for the slopes and intercepts. In this study, each intercept and slope was modeled as random (except for the cross-level interactions), meaning that the entire population of potential exchange responses was not sampled. More specifically, the intent of this study has been to generalize to other levels of exchanges in the population at large. As previously mentioned, all of the Level 1 predictors were person centered; thus, $b_{0 \mathrm{i}}$ can be interpreted as the predicted log expected count of drinking at the person's average level (compared to Tuesday) of the Level 1 predictor. The Level 2 equations are the resulting intercepts and slopes obtained from the Level 1 variables. Finally, because each parameter is modeled as random, it has a variance component, $\mathrm{e}_{\mathrm{it}}$ or $\mathrm{u}_{0}$.

Explanation of coefficients and results are provided below, separately by hypothesis. Day-of-week dummy variables were included (Tuesday was the contrast) and were modeled as fixed effects (Bryk \& Raudenbush, 1992, p. 151).

Hypothesis Tests

In the next section the results from the hypothesis tests are presented. Table 9 
Daily Social Support and Drinking, 95

in this document summarizes each hypothesis and the results of the hypothesis testing.

\section{Hypothesis 1}

In this model, I hypothesized that daily supportive interpersonal exchanges (SIE) would be negatively associated with subsequent drinking at home alone.

$$
\begin{gathered}
\text { DrinkingHome } \mathrm{t}_{\mathrm{t}}=\mathrm{b}_{0 \mathrm{i}}+\mathrm{b}_{1 \mathrm{i}}\left(\mathrm{SIE}_{\mathrm{t}-1}\right)+\mathrm{b}_{2 \mathrm{i}} \text { (Mon) }+\mathrm{b}_{3 \mathrm{i}}(\text { Wed })+\mathrm{b}_{4 \mathrm{i}}(\text { Thurs })+\mathrm{b}_{5 \mathrm{i}}(\text { Fri }) \\
+\mathrm{b}_{6 \mathrm{i}}(\text { Sat })+b_{7 \mathrm{i}}(\text { Sun })+b_{8 \mathrm{i}}(\text { Time-of-day })+\mathrm{e}_{\mathrm{it}} \\
\mathrm{b}_{0 \mathrm{i}}=\gamma_{00}+\mathrm{u}_{0 \mathrm{i}}
\end{gathered}
$$

In the equation for Hypothesis 1, Drink $\mathrm{it}_{\mathrm{it}}$ is person i's log drink count on day $t, b_{0 i}$ is the subsequent predicted value of drink for person $i$ when socially supportive exchanges equaled their person-mean on day $\mathrm{t}-1, \mathrm{~b}_{1 \mathrm{i}}$ is the partial within-person regression coefficient for the predictor for person $\mathrm{i}$, and $\mathrm{e}_{\mathrm{it}}$ is a random residual component.

As hypothesized, there was a significant inverse relationship between socially supportive exchanges and succeeding solitary drinking at home (see Table 5). This indicates that at times when individuals reported more socially supportive exchanges they reported fewer subsequent drinks at home alone compared to times in which they reported fewer socially supportive exchanges. I then exponentiated the logexpected count coefficient (see Table 5) to obtain an interpretable value (exp $\{-.07\}=$ .93). Holding all else constant, for one unit increase in social support, drinking amount decreased by $7 \%$. In other words, consistent with the direct effect model, as 
Daily Social Support and Drinking, 96

individuals reported more daily socially supportive exchanges, they later reported drinking less at home alone.

\section{Hypothesis 2}

Consistent with the stress-buffering model (Cohen \& Wills, 1985), I hypothesized that more daily supportive exchanges would moderate the negative exchange (NIE)-subsequent drinking at home alone relationship compared to times with fewer daily supportive exchanges.

$$
\begin{aligned}
& \text { DrinkingHome }{ }_{\mathrm{t}}=\mathrm{b}_{0 \mathrm{i}}+\mathrm{b}_{1 \mathrm{i}}\left(\mathrm{SIE}_{\mathrm{t}-1}\right)+\mathrm{b}_{2 \mathrm{i}}\left(\mathrm{NIE}_{\mathrm{t}-1}\right)+\mathrm{b}_{3 \mathrm{i}}\left(\mathrm{NIE}_{\mathrm{t}-1} \mathrm{x} \mathrm{SIE}_{\mathrm{t}-1}\right)+\mathrm{b}_{4 \mathrm{it}}(\text { Mon })+ \\
& \mathrm{b}_{5 \mathrm{it}}(\text { Wed })+\mathrm{b}_{6 \mathrm{it}}(\text { Thurs })+\mathrm{b}_{7 \mathrm{it}}(\text { Fri })+\mathrm{b}_{8 \mathrm{it}}(\text { Sat })+\mathrm{b}_{9 \mathrm{it}}(\text { Sun })+b_{10 \mathrm{i}}(\text { Time-of-day })+\mathrm{e}_{\mathrm{it}} \\
& \mathrm{b}_{0 \mathrm{i}}=\gamma_{00}+\mathrm{u}_{0 \mathrm{i}} \\
& \mathrm{b}_{0 \mathrm{i}}=\gamma_{10}+\mathrm{u}_{1 \mathrm{i}} \\
& \mathrm{b}_{20 \mathrm{i}}=\gamma_{20}+\mathrm{u}_{2 \mathrm{i}}
\end{aligned}
$$

In the equation for Hypothesis 2, Drink $\mathrm{it}_{\mathrm{it}}$ was person i's log drink count on day $\mathrm{t}, \mathrm{b}_{0 \mathrm{i}}$ was the subsequent predicted value of drink for person i when all of the predictors equal the person-average on day $\mathrm{t}, \mathrm{b}_{1 \mathrm{i}}-\mathrm{b}_{3 \mathrm{i}}$ are the partial within-person regression coefficients for the predictors, including the moderating variable, for person $\mathrm{i}$, and $\mathrm{e}_{\mathrm{it}}$ was a random residual component.

Thus, I hypothesized that the regression coefficient would be negative, such that as supportive exchanges increased the negative exchange-drinking relationship would decrease). Because this model includes the test of an interaction effect, the 
Daily Social Support and Drinking, 97

coefficients were standardized (please refer to the Interaction subsection of the Data Analysis section on pp. 61-63).

Hypothesis 2 - Step 1. The first step in examining the potential moderating effect was to consider whether negative exchanges were positively related to number of drinks at home alone. Counter to expectations, the slope in this model indicated that there was a significant inverse relationship between negative interpersonal exchanges and later solitary drinking at home (see Table 6a). Holding all else constant, one standard deviation increase in negative exchanges decreased the number of subsequent solitary drinks at home by $19 \%(\exp \{-.21\}=.81)$. Furthermore, given the absence of a positive relationship between negative exchanges and solitary consumption (which is based on the tension-reduction hypothesis), it is improbable that supportive exchanges will serve a function of reducing the positive effect of negative exchanges on consumption.

Additionally, solitary drinks at home were also modeled as a function of socially supportive exchanges, in which case supportive exchanges did not significantly predicted later solitary drinking at home. These results are not consistent with Hypothesis 1 in which tested this direct-effect. It is possible that when controlling for the number of negative exchange, the socially supportive exchange-later solitary drinking relationship is no longer present.

Hypothesis 2 - Step 2. When I did not find the expected effect, one might argue against testing this relationship (Mertler \& Vannatta, 2005). However, it may 
Daily Social Support and Drinking, 98

be that the relationship between negative exchanges and consumption could differ based on the level of supportive exchanges. Taking that in to account, I proceeded with the analyses.

For the next step I modeled the main effects for socially supportive exchanges and negative exchanges as well as the interaction term. In particular, I included the Negative Exchange X Supportive Exchange interaction (see Table 6b, which reflects the interaction term on the lagged exchange-drinking association as depicted in Hypothesis 2). The results revealed that, counter to my hypothesis, social support did not moderate the negative exchange-subsequent drinking association. More specifically, the interaction term was not significant (see Table $6 b)$; thus, Hypothesis 2 was not supported.

In sum, my results were not supportive of the buffering model, wherein supportive exchanges buffer the effects of negative exchanges on solitary drinking at home. At the same time, the absence of a significant moderation effect were not surprising given the inverse relationship between negative exchanges and solitary drinks at home; results which are suggestive that negative exchanges may serve a protective function against solitary drinking behaviors. In a sample of moderate-toheavy drinkers, it is possible that at times when they are experiencing more negativity they are choosing not to drink alone, but instead direct their attention toward addressing the problem more directly (consistent with problem-focused coping; Lazarus \& Folkman, 1984). However, on days when these individuals are 
Daily Social Support and Drinking, 99

not having more negative exchanges (which are most days in this sample) the socially supportive exchanges are having the desired effect (i.e., a direct effect on solitary drinking).

Hypothesis 3: Perceived Support

For this model I hypothesized that individuals with higher levels of perceived social support (PSS) would report less solitary drinking at home compared to those with lower perceived social support across study days.

DrinkingHome $_{i t}=b_{0 \mathrm{i}}+b_{1 \mathrm{it}}($ Mon $)+\mathrm{b}_{2 \mathrm{it}}($ Wed $)+\mathrm{b}_{3 \mathrm{it}}($ Thurs $)+\mathrm{b}_{4 \mathrm{it}}($ Fri $)+\mathrm{b}_{5 \mathrm{it}}($ Sat $)$

$+b_{6 i t}($ Sun $)+b_{7 i t}($ Time-of-Day $)+e_{i t}$

$$
\mathrm{b}_{0 \mathrm{i}}=\gamma_{00}+\gamma_{01}(\mathrm{PSS})+\mathrm{u}_{0 \mathrm{i}}
$$

Solitary drinking at home was regressed on perceived social support (Level2), which was grand mean centered. Interestingly, perceived social support did not significantly predict later drinking, (see Table 7); thus, this hypothesis was not supported.

Thus, despite many benefits documented in the literature between perceived social support and health outcomes (such as the common cold; see Cohen, 2001), perceived support in this study was not related to lower levels of solitary drinks at home.

\section{Hypothesis 4}

I hypothesized that the moderating effects of daily socially supportive exchanges (SIE) on the negative exchange (NIE)-subsequent solitary drinking at 
home relationship would be stronger for those with higher perceived social support compared to those with lower levels of perceived support. Because this model includes a within-person interaction term, all variables were standardized for purposes of analysis.

DrinkingHome $_{i t}=b_{0 i}+b_{1 i}\left(\operatorname{SIE}_{t-1}\right)+b_{2 i}\left(N_{\text {IE }}-1\right)+b_{3 i}\left(N_{\text {IE x SIE }}-1\right)+b_{4 i t}($ Mon $)+$ $b_{5 i t}($ Wed $)+b_{6 i t}($ Thurs $)+b_{7 \text { it }}($ Fri $)+b_{8 i t}($ Sat $)+b_{9 \text { it }}($ Sun $)+b_{10 \text { it }}$ (Time-of-Day $)+e_{i t}$

$$
\begin{aligned}
& b_{0 i}=\gamma_{00}+\gamma_{01}(\text { PSS })+u_{0 i} \\
& b_{10 i}=\gamma_{10}+\gamma_{11}(\text { PSS })+u_{1 i} \\
& b_{20 i}=\gamma_{20}+\gamma_{21}(P S S)+u_{2 i} \\
& b_{30 i}=\gamma_{30}+\gamma_{31}(P S S)+u_{3 i}
\end{aligned}
$$

Step One. First, I ran the model with cross-level interactions but without the three-way interaction term (i.e., NIE x SIE x Perceived Social Support); more specifically, I regressed solitary drinking at home on my Level 1 (negative and socially supportive exchanges) and Level 2 (perceived social support) variables (see Table 8a).

Interestingly, perceived social support did not moderate the socially supportive exchange-subsequent solitary drinking at home relationship in this model (SIE x Perceived Social Support). Conversely, perceived support did, in fact, moderate the negative exchange--subsequent drinking association (NIE x Perceived Social Support). Following the recommendations from Aiken and West (1991) for interpretation of this interaction effect, I conducted a simple slopes analysis of this 
Daily Social Support and Drinking, 101

relationship. More specifically, I created high and low values of perceived support (+/- 1 standard deviation from the mean) to probe the negative exchange-drinking relationship at higher vs. lower levels of perceived support. This allowed me to examine whether the negative exchanges were significantly associated with solitary drinking for those who were higher and those who were lower on social support (Preacher, 2003).

Results revealed that at higher levels of perceived support, the negative exchange-drinking relationship was significant and negative $(\mathrm{b}=-.17, p=.02)$. The slope was also significant for those with lower levels of perceived support $(b=-.38$, $p<.001)$. For additional interpretation, I graphed the model based on the procedure put forth by Aiken and West (1991) in which I used simple slopes to plot simple regression equations (see Aiken \& West, 1991, p. 52). As revealed in Figure 7, people with lower levels of perceived social support demonstrated a stronger negative exchange-solitary drinking at home relationship compared to those with higher levels of perceived support. In other words, for those with higher levels of perceived support, increases in negative exchanges reduced subsequent solitary drinking but not as strongly as for those with lower levels of perceived support.

Step Two. Next, the within-person interaction term was added to the model. Similar to the previous model (Step One), for individuals with higher levels of perceived support, there was a significant positive association between negative exchange and later drinking compared to those with lower levels of perceived 
Daily Social Support and Drinking, 102

support, $(\mathrm{b}=.12, p<.01)$. Interestingly, there was not a significant relationship between supportive exchanges and subsequent drinking for those who were higher on perceived support compared to those who were lower on perceived support $(b=-$ $.03, p=.20)$.

Finally, I hypothesized that the moderating effects of daily socially supportive exchanges (SIE) on the negative exchange (NIE)-subsequent solitary drinking at home relationship would be stronger for those with higher perceived social support compared to those with lower levels of perceived support; my results did not support this hypothesis. Results are presented in Table $8 b$ under Step Two.

In sum, the interaction between perceived support on the moderating effects of daily support on the negative exchange-subsequent drinking relationship was not significant. Interestingly, higher levels of perceived support moderated the negative exchange-drinking association in step one and two of this model.

\section{Summary}

A summary of all the hypothesis tests are presented in Table 9 at the back of this document. In sum, I found that Hypothesis 1 was supported; specifically, I tested the direct-effect of social support on solitary drinking at home. Results revealed that there was a significant inverse relationship between socially supportive exchanges and subsequent solitary drinking at home. This suggests that at times when individuals reported more socially supportive exchanges they later reported fewer 
Daily Social Support and Drinking, 103

drinks at home alone compared to times in which they reported fewer socially supportive exchanges. These results are presented in Table 5 in this document.

For Hypothesis 2, I examined whether socially supportive exchanges buffered the negative exchange-later drinking relationship. Counter to the hypothesis, there was a significant inverse relationship between negative interpersonal exchanges and later solitary drinking at home. Interestingly, socially supportive exchanges did not have a significant relationship with drinking at home alone and supportive exchanges did not buffer the negative exchange-drinking relationship. These results can be found in Tables $6 \mathrm{a}$ and $6 \mathrm{~b}$.

Hypothesis 3 examined whether individuals with higher levels of perceived social support (PSS) would report less solitary drinking at home compared to those with lower perceived social support across study days. Results did not support this hypothesis. Table 7 reflects these findings.

Finally, for Hypothesis 4 I predicted that the moderating effects of daily socially supportive exchanges on the negative exchange -subsequent solitary drinking at home relationship would be stronger for those with higher perceived social support compared to those with lower levels of perceived support. I ran this model in two steps; one that included the between and within-person predictors but no within-person interaction term and one model that included all the predictors and the interaction term. In Step One, results revealed that at higher levels of perceived support, there was a negative and significant negative exchange-drinking 
Daily Social Support and Drinking, 104

relationship. To further probe this associate I graphed the simple slopes. Figure 7 were that people with lower levels of perceived social support had a stronger negative exchange-solitary drinking at home relationship compared to those with higher levels of perceived support. In Step Two of this model I added the withinperson interaction term and found that for those with higher levels of perceived support (compared to those with lower levels of perceived support) there was not a significant moderating effect of daily socially supportive exchanges on the negative exchange (NIE)-subsequent solitary drinking at home relationship. Those results are offered in Table $8 b$ under Step Two.

Therefore, as depicted in Table 9, Hypothesis 1 was supported but Hypothesis 2, 3, and 4 were not. In the following Discussion chapter, I will discuss my findings in light of relevant theories and current research. 
Daily Social Support and Drinking, 105

\section{CHAPTER 8}

\section{Discussion}

This dissertation attempted to expand our understanding on how daily social support may have a direct and a stress-buffering effect on tension-reduction drinking, specifically on solitary drinking at home. Even though the stress-buffering hypothesis has demonstrated how social support moderates potential negative effects of stressors on outcomes such as depression (Burks \& Martin, 1985), chronic illness (Sarason, Sarason, Potter \& Antoni, 1985), and anxiety (Wethington \& Kessler, 1986), only a modest amount of research has examined support as a buffer against stress-related drinking. Even less research has examined the possible direct effect that social support may have on drinking behaviors (i.e., drinking at home alone) considered to be maladaptive. This is regardless of the overwhelming acknowledgment of the importance of social support in drinking interventions and treatment (e.g., Alcoholics Anonymous; Eckenrode \& Hamilton, 2000). Thus, this empirical research project provided evidence about whether the direct and buffering effects of daily social support influence solitary drinking at home in a moderate-toheavy drinking sample.

To further understand solitary drinking at home, social support and the transactional process in which the interaction between the two unfolds, I adopted research methods that other researchers have employed this phenomenon (i.e., daily process methodology; Reis \& Gable, 2000). While the benefits will be discussed 
Daily Social Support and Drinking, 106

later in this section, it is important to note that one noted benefit of capturing life as it unfolds (i.e., daily process strategies) is that researchers do not need to rely on long term retrospection from participants, which is often deemed as unreliable (Bolger, et al., 2003; Mohr et al, 2003). Although retrospective data collection methods are psychologically important they are not able to capture the dynamic interplay between interactions and (current and subsequent) behaviors. Daily methodology allows researchers to capture information that has not been changed with time. Additionally, in this study, data were not collected only daily, but also multiple times per day. This type of data collection required minimal retrospection from the participants and is consistent with the recommendation from Perrine et al. (1994) to obtain accurate accounts of alcohol consumption it is necessary for participants to record the information within 24 hours of consumption. Researchers have argued that if the goal is to examine interpersonal event-related drinking then daily process methods are necessary (Hussong, 2007; Mohr et al., 2005; Swendsen, Tennen, Carney, Affleck, Willard, \& Hromi, 2000). Consequently, this study followed the advice from previous work and implemented such methods.

In this discussion, I have summarized the results of Hypotheses 1 through 4 individually. First, I examine the direct effect of daily social support on subsequent drinking at home alone (Hypothesis 1). Second, I examine whether daily supportive exchanges moderate the negative exchange-subsequent drinking relationship; in other words, I examine the stress-buffering model of support on later drinking 
Daily Social Support and Drinking, 107 behaviors (Hypothesis 2). Third, I discuss whether individuals with higher levels of perceived support report less drinking over this 30 day study compared to those who had lower level of social support (Hypothesis 3). Lastly, I offer information on a complex multilevel model that examined how (high or low) levels of perceived social support might influence the moderating effects of socially supportive exchanges on the negative exchange-drinking relationship (Hypothesis 4). After I summarize the study findings, I review the strengths and limitations of this study as well as its implications for future research.

\section{Direct-Effect Model}

Although previous research has examined the effects of social support on drinking related outcomes (e.g., Pierce et al., 1996), no research, to my knowledge, has examined the main effects of daily social support on subsequent solitary drinking at home. According to the direct-effect model of social support (Cohen \& Wills, 1985) argue that having relationships has an advantageous effect on health and wellbeing. More specifically, being socially integrated allows an individual to have higher levels of perceived and received support, which results in better health behaviors (Cohen \& Wills, 1985; Rodriguez \& Cohen, 1998). Using daily process methodology, Hypothesis 1 offered a unique test of the direct-effect model and provided greater understanding of how our daily interactions with others affect solitary drinking at home behaviors. 
Daily Social Support and Drinking, 108

My findings revealed that at times when the participants reported more positively appraised, socially supportive exchanges, they reported less drinking at home alone in the next time period relative to times when they reported fewer positive socially supportive exchanges. This is consistent with Rodriguez and Cohen (1985) who argued that positive interpersonal exchanges have a direct effect on wellbeing and health regardless of the presence of a stressor. My findings were also consistent with Vittengl and Holt (1998) who examined the relationship between daily social support and mood cross-sectionally within-day. They found that daily social support directly affected (i.e., enhanced) positive mood. Moreover, my results were consistent with research conducted by Gable and colleagues who found a direct effect of daily exchanges and mood (Gable et al., 2004). More specifically, they found that on days in which individuals reported sharing a positive experience with other individuals they also reported experiencing significantly higher positive mood and life satisfaction (Gable et al., 2004).

Because I person-centered the data I was able to determine whether following times in which people experienced more support they were drinking more (or less) than their normal (average) behavior. Importantly, because I examined the relationship between socially supportive behaviors and subsequent alcohol using daily methodology, I was able to determine the temporal association between my predictor and outcome variables (Bolger, Davis \& Rafaeli, 2003). This is a necessary precondition in establishing causality and for internal validity (Cook \& Campbell, 
Daily Social Support and Drinking, 109

1973). Although I cannot eliminate all plausible explanations, I can determine that the exchanges (i.e. predictors) occurred before people drank (i.e., outcome). This is a benefit of this type of methodology.

With evidence of this temporal relationship, my results also indicate that socially supportive exchanges, even if relatively mundane, have more than a momentary impact on individuals. I suggest that the direct effect of social support on drinking is consistent with the Fredrickson and Joiner's (2002) broaden and build argument. They argue that positive emotions can "spiral upwards" and it is possible that one positively appraised interaction could start this upward spiral, building on positive moods which results in higher levels of overall well-being. It is reasonable to suggest that one positively appraised supportive interaction could have the same effect, which results in a reduced need to drink-to-cope.

Another potential explanation of my results is that when people are experiencing a lot of support they may want to share that elevated affective state with others (Reis et al., 2010), in other words, to capitalize on their positive state (Gable, et al., 2004); this is not a process of which solitary drinking is a part. Research by Gable and colleagues has demonstrated that when good things happen people react by sharing (Gable et al., 2004). Engaging in social comparison and sharing with other when good things happen (i.e., capitalization) was related to higher levels well-being, higher levels of intimacy and marital satisfaction (Gable et al., 2004). Future research should probe whether or not drinking plays a role in the 
capitalization process. It is possible that at times when participants in my study reported more supportive exchanges they were capitalizing by drinking away from home with others, or exercising with friends after work, or enjoying a quiet meal with their partner. Future research should probe these alternative contexts more fully to gain an understanding of this direct effect process.

One caveat to the interpretation of my direct effect results is the fact that the relationship was not significant in my test of Hypothesis 4. However, as noted earlier, there were important differences in these two models; chief among them is the fact is that negative exchanges were also entered into the model of Hypothesis 4. Previous similar studies have typically found that when negative and positive events were both entered in to the models as predictors of solitary consumption, positive events did not predict drinking outcomes (Mohr et al., 2001). It appears that in a sample like mine in some ways the negative events/exchanges may be having a stronger effect than the positive (when they occur). Given that in my study supportive exchanges are happening more often than negative (as positive events typically are), the direct-effect of supportive exchanges is likely occurring with greater frequency than the reducing effect of negative exchanges.

In sum, because of the unique methodology in this study (e.g., signal contingent data collection) I was able to examine temporal relationships between supportive exchanges and solitary drinking at home. More specifically, I was able to determine that supportively supportive exchanges clearly preceded solitary drinking 
Daily Social Support and Drinking, 111

at home. This dissertation has examined two important processes (social support and solitary drinking) in a way that has not been done before; thus, contributing to our understanding of how daily events affect behaviors.

\section{Stress-Buffering Model}

My second hypothesis was based on the stress-buffering model of social support, which posits that received support can reduce the impact or even counteract the effects of stressors on mental and physical health (Cohen et al., 2000). Cohen and Wills (1985) argued that when individuals experience stressors, the receipt of tangible support resources (i.e., received support), will reduce the negative effects of stressors (Cohen, et al., 2000; Cutrona, 1986; Thoits, 1986). Pierce et al. (1996) suggested that the buffering effect of social support disrupts the drinking-to-cope motives as well as offers resources that are needed during stressful periods (Pierce et al., 1996). Based on this model, I expected daily socially supportive exchanges to moderate the negative exchange-subsequent drinking relationship. Surprisingly, the results did not support this hypothesis. Specifically, supportive exchanges did not moderate the negative exchange-solitary drinking at home relationship.

Yet, much of the research on the stress-buffering model of social support has produced inconsistent results. It has been argued that the inconsistencies have been attributed to the fact that the supportive exchanges that were provided were done so with the intent to help (e.g., buffer) but they were often appraised by the beneficiary as being unhelpful and stressful (Cohen \& McKay, 1984; Cutrona, 1990; Rook, 
Daily Social Support and Drinking, 112

1984; Coyne et al., 1988). To address this issue, I only included positively appraised supportive exchanges. However, there were no significant buffering findings. As with negative exchanges, the magnitude of the appraisal may be key; more specifically, it may be that the supportive exchanges needed to be very positive to buffer individuals from negative events.

It is also important to recognize that there is a cognitive component to the stress-buffering model. Negative interpersonal exchanges could be appraised as less negative if a positive exchange happened before a full appraisal of the negative event occurred. Returning to the transactional model of stress and coping, individuals need to first appraise the event as negative, then they must decide how to deal with the negative event (e.g., secondary appraisal process; Lazarus \& Folkman 1987). Such a relationship could not be revealed in my analyses. This notion would be consistent with Wheaton's (1985) stress deterrent model described earlier, whereby support prevents the experience of stress. This potential is bolstered by the fact that over the course of this study, only 149 exchanges were considered very negative or extremely negative. Given the high levels of supportive exchanges (1484 were reported as being very positive or extremely positive) it may be that these buffered the primary appraisal process, resulting in fewer extremely negative experiences. Subsequent work should also examine whether supportive exchanges moderate negative exchange appraisals, as opposed to only exchange frequencies, as was done in the present study. 
An additional possibility is that individuals who experience a number of positive, supportive exchanges throughout their day are likely in a more constant positive emotional state (Fredrickson \& Joiner, 2002). Therefore, when these individuals experience a potentially negative interaction or event, rather than experiencing a "fight or flight" reaction, they are able broaden their thinking (i.e., thought-action repertoire) and draw from their potential resources (i.e., social support). As a result, the impact of the negative exchanges and events are reduced (Fredrickson, 2000). This potential buffering effect is not the result of concurrent positive interpersonal exchanges (as measured in the current study) but from an accumulation of positive interpersonal exchanges over the course of days, weeks or even months. In other words, rather than staying focused on a negative event, individuals who experience an abundance of positive exchanges may broaden their minds and think about potential resources that they have available, reappraise events, and engage in proactive coping behaviors.

An alternative explanation may have been that positive exchanges buffer extremely negative or severe stressors, but not mundane everyday experiences. Events could be appraised as negative but they have very little lasting impact on the person because they are commonplace (Reis \& Gable, 2000; Vaux, 1988). For example, it may be common for a person who works in retail to get yelled at by customers and because of this expectation the incident does not cause lingering negative mood. Using the transactional model of stress and coping as a guide, 
Daily Social Support and Drinking, 114

another way to consider this possibility is that a negative (commonplace) "threat" is a transaction between the person and their environment. If an individual is consistently experiencing and appraising low-level threats, in an environment where they expect to experience them from time to time, it is likely that the threat will have a minimal impact and be appraised as more mundane (Vaux, 1988). Moreover, just being a part of a stable social network may reduce the likelihood of perceiving events as "threats." Research by Cassell $(1974,1976)$ has shown that social groups serve a protective function (e.g., buffer against stressors and life events). His research, along with others, has shown that having a stable, secure social network reduced perceived stress and increased health and well-being (Cassell, 1976; House et al., 1988); thus, reducing interpersonal "threats." Accordingly, mundane daily events may not have a large impact on individuals if a) they are used to experiencing those types of events; and b) they are part of a strong social network that help buffer them from the impact of stressors.

Consistent with this argument, some theorists have put forth that each individual has a "set point" for mood and well-being and while daily events and interactions might increase or decrease moods and well-being, people generally return to their set points (Brickman, Coates, \& Janoff-Bulman, 1978; Diener, Lucas, \& Scollon, 2006; Heady, 2008). Research suggests that each person's set-point is based on personality traits and socialization (Diener \& Diener, 1996; Heady, 2008). Additionally, Diener and Diener (1996) found that for most people, their set-point 
Daily Social Support and Drinking, 115

was more positive than negative. Research has also found that even when significant life events occur individuals eventually returned to their "set point" (Diener et al., 2006); thus suggesting that people, in general, return to a more positive psychological place, especially those who are not depressed (depressed individuals were screened out of this study). Moreover, this could be an explanation as to why our participants reported more daily supportive (positive) exchanges compared to negative exchanges. It may be that they were generally happy individuals and they did not need to be "buffered" from daily stressors, particularly mundane ones. Future buffering research may want to study a group of individuals, who may be actively attempting to manage significant life events, such as people who are dealing with chronic illness or severe depression. While research has examined those groups, it may be the information that can be gleaned from multiple surveys in a day that offer the best information. Understanding temporal associations may be the key (Bolger et al., 1998; Vittengl, \& Holt, 1998). In addition, future research that probes daily exchanges should also examine overall happiness and personality traits considering the work that has already shown the significant influence on these variables on health and well-being. Both can have a direct effect on how individuals view difficult and stressful times (Diener \& Diener, 1996).

While using daily methodology in which I am collecting data at multiple time points is ideal for addressing these research questions, it is also important to consider that I may not have used the optimal time frame. It is possible that buffering was 
Daily Social Support and Drinking, 116

happening so quickly that I was unable to capture that information on the diary. The positive exchanges experienced at the time of the negative event could have had a significant and immediate impact on the event. Mohr et al. (2003) also suggested that the impact of negative interpersonal exchanges on the individual is brief; therefore those exchanges did not significantly affect the individual for very long. It is possible that I was not able to detect fluctuations in negative exchanges across interview times because people forgot that they happened. For example, if someone had a negative exchange when they first arrived to work they may have already forgotten about it by the time they take the afternoon survey (between 4:00 - 5:30 p.m.). Further, lagged effects are much less common and more difficult to detect (Bolger et al., 1998; Mohr et al., 2003). Future research is needed to probe alternative temporal models. At the same time, the timing of the current study was intended to capture relationships that coincide with naturally occurring event patterns, such as work-tofamily spillover (e.g., Hanson et al., 2006).

A more fundamental problem to my test of the stress-buffering hypothesis was the revelation that negative exchanges were inversely related to solitary drinking at home. Thus, my participants drank less in that context following times of increases in negative exchanges. This runs counter to motivational models of consumption arguing that people drink more at times when negative or stressful experiences are higher and may be particularly likely to do so in more solitary contexts (Cooper, et al., 1995). This maladaptive coping behavior has been linked to many negative 
Daily Social Support and Drinking, 117

consequences such as decrease in job-related self-image and an increase in work or family problems (i.e., Wang et al., 2010). I posited that the participants in this study would engage in a form of drinking-to-cope (i.e., tension-reduction drinking) when they experienced more negative exchanges. This is surprising given other daily diary studies that have found a significant within-person association between negative experiences and alcohol consumption (e.g., Carney et al., 2000; Mohr et al., 2001; Mohr et al., 2005). It is possible that our results were not the same because many of these studies used concurrent variables that were aggregated on a daily level; conversely, my exchange and drink variables, were collected multiple times each day and not aggregated over the course of the day. One could argue that the way my variables were constructed allowed for a more accurate reflection of the temporal association of key variables; but, unfortunately, it also reduced the statistical power of my analyses relative to the method employed by Mohr et al. (2001). Previous research has posited that lagged effects are much less common and harder to detect (Bolger et al., 1998; Mohr et al., 2003). Thus, it may be that because of the examination of (only) lagged effects, I was unable to capture important information. Another difference between the present study and previous studies on the topic is the type of drinker included in the sample. All of these previous adult studies are of moderate-to-heavy drinkers, for example, the Carney et al. (2000) and Mohr et al. (2001) studies stipulated a higher level of drinking (i.e., no less than 12 drinks a week for women and no less than 15 drinks a week for men) than the present study. 
Daily Social Support and Drinking, 118

Some researchers have found that individuals lower on drinking-to-cope motives do not show the same patterns as problem drinkers (Carney et al., 2000; Todd et al., 2003; Mohr et al., 2001). Conversely, Mohr and colleagues found that individuals who were higher on drinking-to-cope motives drank more in all contexts when compared to those who were lower on drinking-to-cope motives (Mohr et al., 2001). Reported levels of drinking-to-cope motivations were relatively low in the present study (Mohr, 2007) compared to these other studies. Indeed, Todd, Armeli, Tennen, Carney and Affleck (2003) revealed that for those with lower levels of drinking-tocope motivations, the relationship between negative experiences and consumption is typically an inverse one, such that those individuals tend to drink less when their negative experiences increase. As Todd et al. (2003) suggested it is possible that "problem-drinkers" (i.e., alcohol abusers or alcohol dependent individuals) are the group that engages in coping-related drinking and our sample did not comprise those types of drinkers. Future research examining stress-buffering on alcohol consumption should consider probing a larger sample with a broader array of drinkers, including alcohol abusers, for whom such buffering effects may be more evident.

In sum, Hypothesis 2 was not supported, wherein no evidence of stressbuffering was found. The absence of a relationship between negative exchanges and subsequent increases in solitary drinking at home proved highly problematic in terms of establishing stress-buffering of social support. As I will return to in a later section, 
Daily Social Support and Drinking, 119

these results highlight the need for a reexamination of behaviors that theorists define as maladaptive (i.e., solitary consumption).

\section{Perceived Social Support}

Researchers have argued that perceived social support can be considered a “psychological sense of support” (Gottlieb, 1984), meaning that individuals know that they are important to others and they have people willing to help in times of need (Heller, et al., 1986; Gottlieb, 1984). Perceived social support also has been implicated in the stress and coping process. Individuals' perceived levels of support can influence the appraisal process whereby potential stressors are appraised as more controllable and less threatening due to the supportive resources that they believe that they have from people in their social network.

In this dissertation, one of my goals was to examine the relationship between perceived support and solitary drinking at home. I hypothesized that individuals with more perceived support would report less solitary drinking at home across the study compared to individuals with less perceived support. Results indicated that there was no relationship between perceived support and solitary drinking. A review of the perceived support levels in this study suggests that my sample reported comparable levels of perceived support compared to other samples of adults (see http://www.psy.cmu.edu/ scohen/), yet in this study there was insufficient variability to detect meaningful associations with daily solitary drinking at home. Given that there were a number of exclusion criteria in this study, including 
Daily Social Support and Drinking, 120

depression and anxiety, it is reasonable to conclude that this was a small and relatively homogeneous sample of people; thus significant results could not be detected. A larger, more diverse sample may enable researchers to shed light on the possible relationship between perceived support and solitary drinking at home because of the greater statistical power. In addition, future studies should specifically recruit samples that are more diverse in nature. Although this sample had some ethnic diversity, the majority of the sample was European-American (90\%). Considering previous research has demonstrated significant differences in drinking levels, consumption over time, and health outcomes when comparing the AfricanAmericans and Hispanic populations to Caucasian samples (see Koniak-Griffin, Lominska, Brecht, 1993; Norbeck \& Anderson, 1989) it is important to point out that this is a potential limitation of this study. Previous research has revealed that NonCaucasian drink less alcohol than Caucasians, but they report more problems associated with drinking such as racial and ethnic stigma consciousness, being treated unfairly, poverty and psychological distress (Mulia, et al., 2008). While there have been studies examining the differences in support among different ethnic groups (see Koniak-Griffin et al., 1993; Norbeck \& Anderson, 1989). I am not aware of a study examining daily drinking patterns as a function of ethnicity, and so it may be important for future research to probe possible differences in daily social support and tension-reduction drinking behaviors in a sample that includes racial/ethnic minorities. 
Daily Social Support and Drinking, 121

Hypothesis four offered additional information about the function of perceived social support, in that I considered whether the moderating effects of supportive exchanges on the negative exchange-subsequent solitary drinking at home relationship was stronger for those with higher perceived social support. Given the absence of the hypothesized moderating effect of supportive exchanges on the negative exchange-solitary drinking relationship, it was not probable that this hypothesis would be supported; indeed, hypothesis four was not supported. As such, many of the points made for the stress-buffering model could hold true for this model. Most importantly, negative exchanges were associated with a reduction in subsequent solitary drinking at home. Results from testing hypothesis four revealed that this reduction was stronger for those who have lower levels of perceived social support (relative to those with stronger social support). That is, those who have higher levels of perceived support demonstrated a significant but smaller effect of negative exchanges on their solitary consumption. This suggests a reduced level of reactivity to the negative exchanges. This is consistent with previous research that has shown that women, in particular, who have higher levels of perceived support have lower levels of neuroticism and higher levels of adjustment (Sarason, Levine, Basham, \& Sarason, 1983). Overall, my results support the notion that solitary alcohol consumption should be reconceptualized as a drinking behavior that is not necessarily maladaptive, a point I now turn to. 
Daily Social Support and Drinking, 122

\section{Implications of Dissertation}

First, in terms of the outcome variable of solitary alcohol consumption, motivational models of alcohol consumption have posited that people are motivated to drink as a method of coping and are especially likely to do so in solitary drinking contexts (Cooper et al., 1995). This motivational pathway is derived from the tension-reduction hypothesis (Conger, 1956); hypothesizing that people drink to reduce anxiety and stress caused by negative experiences and situations, and the resulting reduction in tension reinforces the drinking behavior. As previously noted, many daily process studies have documented just such a relationship (e.g., Mohr et al., 2001). Yet, we were unable to find a positive relationship between negative experiences and solitary consumption in the present study, instead finding the opposite, wherein negative experiences are related to less solitary consumption. Many potential interpretations of these findings have already been discussed

I would like to put forth the argument that solitary consumption should not be uniformly regarded as a maladaptive behavior; this is in contrast to current interpretations in which it drinking is often treated as either an indicator of current alcohol problems or a signal for future potential problems.

First, research examining work-family conflict suggests that after stressful days, taking time to withdrawal from social interactions and regroup may actually be beneficial (Repetti, 1992). Stress can result in physical and mental fatigue and research has shown that taking the time to remove oneself from a stress and to have 
Daily Social Support and Drinking, 123

solitude, time to regroup and reenergize, can significantly enhance mood (Larson \& Csikszentmihalyi,1983; Repetti, 1992; Thayer, 1989). It is reasonable to suggest that for some individuals coming home and having some solitude and a glass of wine or beer may be what they do to recover from a stressful day. Importantly, I argue that this solitary drinking behavior (which is related to coping according to the motivational models alcohol consumption; Conger, 1997) actually may not be “coping" per se but rather a time to unwind and shift from one role to another. The end result of this activity could be a reduction in stress.

Also in support of my argument is evidence from other analyses of the current data set revealing healthier outcomes associated with negative experiencesolitary drinking relationships (Mohr et al., 2010). Specifically, we used data from this daily study to predict health and well-being outcomes 12 months later. Results demonstrate that those with stronger negative experience-solitary consumption relationships (compared to participants with weaker relationships) reported higher levels of satisfaction with life 12 months later. These results support the notion that those who do engage in tension-reduction drinking do not suffer subsequent problems (i.e., lower levels of satisfaction with life; higher levels of drinking to cope behaviors), at least in a sample of moderate to heavy drinkers that are psychologically well-adjusted.

In sum, solitary drinking is not necessarily a sign of maladaptive coping and this study supports that notation. In addition, in a sample such as mine, the negative 
Daily Social Support and Drinking, 124

exchange-drinking pattern is also not necessarily problematic. Moreover, it is important to point out that current research has examined whether to expand the recommended guidelines for drinking because the USDA health guidelines of 1 drink per day for women and 2 per day for men is considered too conservative (Dawson, 2010). In addition, people who are drinking higher levels than this recommended amount are not necessarily showing symptoms of alcohol or health related problems. Future work should focus on identifying where the cut point between what is healthy and what is not lies.

The results of this study indicate that in a healthy, non-problem sample, received support that is positively appraised directly reduces solitary consumption. This is a significant finding considering that received support is difficult to document. Simultaneously, my results revealed that negative exchanges may have a stronger direct effect than positive exchanges on a reduction in solitary consumption, at least among this type of drinker (i.e., moderate-to-heavy). Moreover, as previously mentioned, individuals in this study may have been engaged in other activities instead of staying home and drinking by themselves, after experiencing negative exchanges.

Furthermore, in a non-clinical sample, the relationship between social support and drinking is not straightforward. Drinking may play an important role in the support-seeking process. It is possible that many supportive interactions involve alcohol (i.e., having dinner with someone; meeting for drinks after work). I argue 
Daily Social Support and Drinking, 125

that it is necessary for social support researchers to develop a more nuanced perspective on the link between support and alcohol consumption, which includes many different kinds of contexts in which support may be operating.

\section{Limitations}

Whereas a number of strengths and limitations were highlighted in this discussion section is important to point out other limitations of this study. In Chapter 2 the metaconstruct of social support was presented which includes perceived and received support and social network support. Unfortunately, this study only examined perceived and received support because no social network variables were collected on a daily basis; thus, it was not possible to test every part of the metaconstruct of social support. It is reasonable to suggest that important support provider data are missing. House (1981) speculated that to comprehend social support we need to identify and understand what resources are being provided as well as who is providing support. Without network information, a key aspect of social context was missing which may moderate associations of interest in this study. However this is the first study to examine within-day support and tension reduction drinking behaviors and it is a profound first step in understanding that relationship. Another limitation is that the handheld electronic interviewer was programmed with preselected supportive interpersonal exchanges and negative exchanges. Although extensive effort went in to creating the list of supportive and negative exchanges, it is likely that the handheld interviewer questions did not 
Daily Social Support and Drinking, 126

capture all the possible daily supportive and negative exchanges. Although not every possible exchange was captured, the items that were on the handheld interviewer were likely highly representative of daily support and negative exchange since extensive research went into determining the positive exchange checklist (Mohr \& Brannan, unpublished manuscript). Future work may want to capture qualitative data through paper and pencil diaries or voice activation recorder on the palm computer to further probe daily exchanges. This would enable participants to record information about exchanges not on the handheld interviewer.

Furthermore, this study was part of a larger study examining moderate-toheavy drinkers; abstinent and light drinkers were excluded, as were abusers or dependent drinkers. Based on previous research it is reasonable to suggest that problem drinkers (i.e., binge drinkers) are significantly different than this group of moderate-to-heavy drinkers (see Wechsler, Dowdall, Davenport, \& Rimm, 1995; Wechsler \& Nelson, 2001); thus, generalization is not recommended. More specifically, when examining previous research it becomes apparent that that abusive and dependent population (e.g., Cooper, Russell, \& George, 1988; Mohr et al., 2001), have lower levels of social support and well-being compared to a sample such as this one (e.g., no mental or physical health issues). It is also important to point out that little is known about the relationship between social support and those who are abstinent or light drinkers. Thus, it is difficult to assess how the current sample might be different. Yet in an unpublished random sample survey conducted 
Daily Social Support and Drinking, 127

by Mohr and Brannan (unpublished manuscript) which included respondents across the drinking spectrum, perceived social support was unrelated to drinking level.

In addition, although this study obtained information about general employment information such as occupational field and whether an individual worked full or part time, future research should probe when individuals work. With the advent of the home office and ever increasing shift work (Beers, 2000) more people are choosing to work at home; thus, the 9-5 work day does not exist or apply for many (Beers, 2000). Thus, future research that probes the drinking behaviors of individuals who work from home or are shift workers may be advantageous in helping to clarify daily drinking behaviors.

While daily process designs have many benefits, one potential concern that has been brought forth by researchers (see Bolger, 1989; Mohr et al., 2009) addresses the issue of reactivity. A logical area of concern in these types of methods is the extent to which an individual is influenced by recording their own behavior (e.g., Affleck, Zautra, Tennen, \& Armeli, 1999). Although using daily diaries has been used as a method of therapy, examination of such trends has not supported this concern (Affleck et al., 1999; Marco, Neale, Schwartz, Shiffman, \& Stone, 1999). The key to reducing reactivity in electronic diaries seems to be to have participants record their information without allowing them to examine their own responses (Hayes \& Cavior, 1980). Moreover, researchers suggest that having participants in daily studies record multiple moods and behaviors also reduce reactivity (Hayes \& 
Cavior, 1980). This study used these guidelines when creating the initial survey; participants were asked about moods, as well as smoking, drinking, sleeping, interaction, to name a few of the behaviors that were probed. In addition, I would like to point out that in the debriefing session $94 \%$ of the participants reported that being in this study did not influence their behavior at all or they were slightly influenced. No participant reported that this study "very much" influenced their behavior. With that said, participants did show a reduction in negative exchanges over the course of the study. It is possible that the diaries had a therapeutic effect on the individuals causing them to reassess and reappraise stressors. As Pennebaker \& Chung's (2007) work has shown, reflecting can assist with adjustment and reappraisals. Future work may consider obtaining qualitative data to try to more fully understand these trends.

Although I used HLM, which is a statistical program that is equipped to handle missing data, missing data was still a concern. It is possible that when people had extreme life events they did not take their daily surveys. While I cannot make assumptions about why people did or did not take the surveys, I am concerned that I might have missed important daily events. It is important to point out that strength of this methodology and specifically, the programming of the handheld surveyor was that it did not allow people to skip questions. Participants were not able to progress until they answered the question(s) on the screen then clicked "next." Once they clicked "next" they were unable to go back to the previous screen. During the initial 
Daily Social Support and Drinking, 129

assessment this point was made to each participant and we asked them to make sure they did not click "next" until they were ready to progress. During debriefing, participants did not mention that they did not like not being able to go back and change questions. In future research, I would like to add a section in the debriefing interview probing why people thought that they missed surveys (i.e., life events got in the way; stress).

Strengths and Future Research

A key strength of this study is that data gathered via daily process methodology was used, thus avoiding some of the methodological issues that arise from cross-sectional and retrospective methods. In addition, the recent interest in the alcohol literature regarding the within-person associations between daily exchanges and alcohol consumption (Carney et al., 2000) suggests that this study may be exceptionally important to the field of alcohol research as well as the area of social support. Each one of these fields is starting to focus more on the temporal associations of interest. This methodology allowed me to examine just that; temporal associations of interest. More specifically, I was able to examine whether negative exchanges predict later drinking or if social support does, in fact, buffer an individual in times of stress.

In addition, another key strength of this study was that it further bridges the gap between the social support and the drinking literature. Few studies have examined the link between social support and alcohol consumption among moderate 
Daily Social Support and Drinking, 130

to heavy drinkers; the majority of all research has examined teens, college students and alcoholics. In other words, the focus has been on those with very high consumption levels. This is unfortunate considering that there are far more people who do not meet criteria for dependence (e.g., moderate to heavy drinkers) but who are considered to be at risk for developing alcohol-related problems (Higgins-Biddle \& Babor, 1996). Moreover, very little research has examined the influence of social support on moderate-to-heavy drinkers and no literature examines the moderating effect of social support on the negative interpersonal exchange -drinking relationship. Consequently, this is an area that is under-examined; thus, this project attempted to fill a research need. In addition, it would be beneficial for future research to build on this dissertation and examine specific daily stressors, not just interpersonal exchanges. Understanding the impact of work stressors on later drinking behaviors could be extremely beneficial.

Future research may want to further probe the influence of marital/partner status on drinking. It seems logical to suggest that drinking "at home" is inherently different for people who have a significant other compared to those who do not. It is possible that an individual who drinks as a way to cope may have to do so in secret or before their partner gets home. Although this study was able to ascertain whether someone was at home drinking while others were in the home or not, qualitative data may shed light what "type" of drinking was occurring. For example, was someone drinking at home, not interacting with others while they were gardening and enjoying 
some quiet time or were they drinking in the garage by themselves because they wanted to escape (drinking-to-cope).

Understanding the influence of personality on the daily-support relationship among moderate-to-heavy drinker could offer a lot of new information to the field. While previous work has examined personality traits such as neuroticism (e.g., Mohr et al., 2005) in conjunction with daily drinking behaviors but to my knowledge no one has examined the influence of personality traits on the daily support-drinking association. It is possible that individuals with higher levels of extroversion may engage in more social drinking because they have more people in their network to drink with. Conversely, it is possible that they are less likely to drink as a method of coping.

In conclusion, this dissertation used daily process methodology to examine the direct-effect of daily social support on solitary drinking at home as well as the possible buffering qualities of social support on the stress-drinking relationship. The social support literature is deeply rooted in the direct-effect model and the stressbuffering model of social support yet no one has yet to examine the influences of these types of daily support on solitary drinking behavior. In an attempt to capture the support- and stress-drinking relationships as it unfolds, data collected via multiple times per day for 30 days. The study sample was comprised of moderate-toheavy drinkers from the local community who responded to 3 interviews per day over 30 days on a handheld electronic surveyor. This dissertation found that daily 
Daily Social Support and Drinking, 132

socially supportive exchanges had significant direct effect on subsequent drinking at home alone. Interestingly, the daily supportive exchanges did not buffer the negative exchanges-later drinking relationship. I also considered the influence of perceived social support on these processes. 
Daily Social Support and Drinking, 133

Table 1. Between-Person Survey Completion Rates

\begin{tabular}{|c|c|c|}
\hline Variable & $\begin{array}{c}\text { Completed 70\% of } \\
\text { all surveys }\end{array}$ & $\begin{array}{c}\text { Completed only 30\% of all } \\
\text { surveys }\end{array}$ \\
\hline $\begin{array}{c}\text { Perceived Social } \\
\text { Support }\end{array}$ & $96 \%$ & $4 \%$ \\
\hline Gender & $100 \%$ & -- \\
\hline Marital Status & $100 \%$ & -- \\
\hline
\end{tabular}

Percentages reflect the ratio of participants who completed either 70 (or more) or $30 \%$ (or less) of the surveys. 
Daily Social Support and Drinking, 134

Table 2. Within-Person Survey Completion Rates

\begin{tabular}{|c|c|c|}
\hline Variable & $\begin{array}{c}\text { Completed 70\% of all } \\
\text { surveys }\end{array}$ & $\begin{array}{c}\text { Completed only 30\% of } \\
\text { all surveys }\end{array}$ \\
\hline $\begin{array}{c}\text { Drinking at Home } \\
\text { Alone }\end{array}$ & $81 \%$ & $2 \%$ \\
\hline $\begin{array}{c}\text { Supportive } \\
\text { Exchanges }\end{array}$ & $68 \%$ & $2 \%$ \\
\hline Negative Exchanges & $68 \%$ & $2 \%$ \\
\hline
\end{tabular}

Percentages reflect the ratio of participants who completed either 70 (or more) or $30 \%$ (or less) of the surveys. 
Daily Social Support and Drinking, 135

Table 3: Frequencies and Percentages for Interpersonal Exchanges

\begin{tabular}{|c|c|c|c|c|}
\hline & $\begin{array}{c}\text { Frequency } \\
\text { of } \\
\text { Negative } \\
\text { Exchanges }\end{array}$ & $\begin{array}{c}\% \text { of } \\
\text { Negative } \\
\text { Exchanges }\end{array}$ & $\begin{array}{l}\text { Frequency of } \\
\text { Supportive } \\
\text { Exchanges }\end{array}$ & $\begin{array}{c}\% \text { of } \\
\text { Supportive } \\
\text { Exchanges }\end{array}$ \\
\hline \multicolumn{5}{|c|}{$\begin{array}{c}\text { Interview } \\
2\end{array}$} \\
\hline 0 & 2373 & 83 & 1785 & 63 \\
\hline 1 & 166 & 6 & 73 & 3 \\
\hline 2 & 90 & 3 & 111 & 4 \\
\hline 3 & 58 & 2 & 122 & 4 \\
\hline 4 & 28 & 1 & 140 & 5 \\
\hline 5 & 23 & 1 & 178 & 6 \\
\hline 6 & 18 & 1 & 157 & 6 \\
\hline 7 & 19 & 1 & 193 & 7 \\
\hline 8 & 18 & 1 & 60 & 2 \\
\hline 9 & 26 & 1 & 0 & 0 \\
\hline \multicolumn{5}{|c|}{$\begin{array}{c}\text { Interview } \\
3\end{array}$} \\
\hline 0 & 2413 & 85 & 1842 & 65 \\
\hline 1 & 130 & 5 & 63 & 2 \\
\hline 2 & 83 & 3 & 96 & 3 \\
\hline 3 & 41 & 1 & 116 & 4 \\
\hline 4 & 43 & 1 & 159 & 6 \\
\hline 5 & 17 & 1 & 176 & 6 \\
\hline 6 & 24 & 1 & 154 & 6 \\
\hline 7 & 16 & 1 & 159 & 6 \\
\hline 8 & 22 & 1 & 54 & 2 \\
\hline 9 & 30 & 1 & 0 & 0 \\
\hline
\end{tabular}


Daily Social Support and Drinking, 136

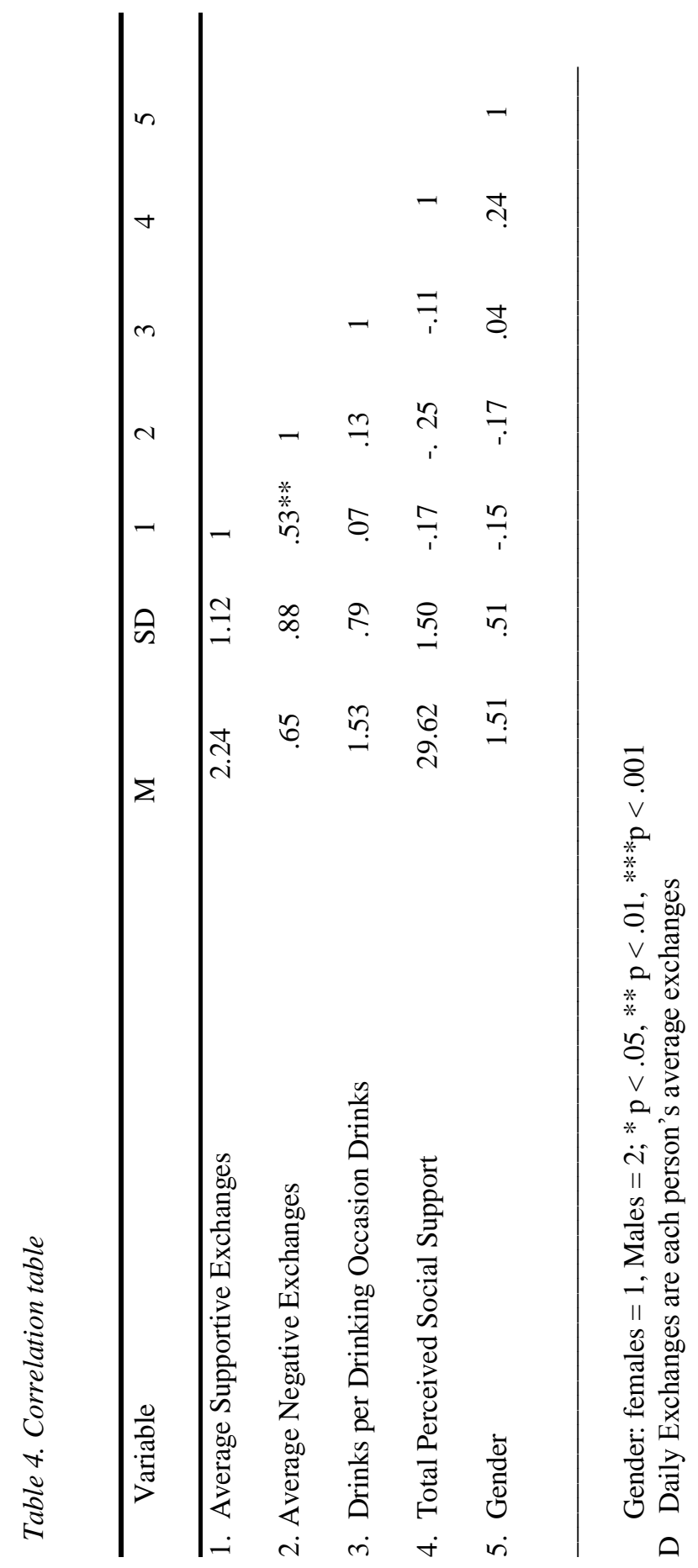


Daily Social Support and Drinking, 137

Table 5. Hypothesis 1, Direct Effect Model

Predictor

Solitary drinking at home at Home

Log Odds Coefficient

Intercept Model

Solitary drinking at home at home

$-1.31 * * *$

Means of SIE

$-.41 *$

Slopes Model

SIE

$-.07 * * *$

Variance Components

Intercept

$1.11 * * *$

SIE Slope

.13

Note: Analyses controlled for day of week and time of day

SIE $=$ supportive interpersonal exchanges

$\dagger p<.10, * p<.05, * * p<.01, * * * p<.001$. 
Daily Social Support and Drinking, 138

Table 6a. Hypothesis 2, Stress-Buffering Model

Predictor

Solitary drinking at home

Log Odds Coefficient

Step One:

Intercept Model

Solitary drinking at home at home

$-1.13 * * *$

Slopes Model

NIE

SIE

$-.08$

Variance Components

Intercept

$1.39 * * *$

NIE Slope

SIE Slope

.12

Note: Analyses controlled for day of week and time of day

$\mathrm{NIE}=$ negative interpersonal exchanges

SIE $=$ supportive interpersonal exchanges

$\dagger p<.10, * p<.05, * * p<.01, * * * p<.001$ 
Daily Social Support and Drinking, 139

Table 6b. Hypothesis 2, Continued

Predictor

Solitary drinking at home

Log Odds Coefficient

Step Two:

Intercept Model

Solitary drinking at home at home

$-1.12 * * *$

Slopes Model

NIE

$-.17 *$

SIE

$-.09$

NIE x SIE (interaction term)

$-.06$

Variance Components

Intercept

$1.40 * * *$

NIE Slope

.06

SIE Slope

.02

Note: Analyses controlled for day of week and time of day

$\mathrm{NIE}=$ negative interpersonal exchanges

SIE = supportive interpersonal exchanges

$\dagger p<.10, * p<.05, * * p<.01, * * * p<.001$ 
Daily Social Support and Drinking, 140

Table 7: Hypothesis 3, Perceived Support

Predictor

Solitary drinking at home

Log Odds Coefficient

Step One:

Intercept Model

Solitary drinking at home at home

$-1.22 * * *$

Slopes Model

Perceived Social Support

$-.07$

Variance Components

Intercept

$1.34 * * *$

Note: Analyses controlled for day of week and time of day

$\dagger p<.10, * p<.05, * * p<.01, * * * p<.001$ 
Table 8a: Hypothesis 4

Predictor

Solitary drinking at home

Log Odds Coefficient

Step One:

Intercept Model

Solitary drinking at home

$-1.10 * * *$

Perceived Social Support

$-.20$

Slopes Model

NIE

NIE X Perceived Social Support

SIE

$-.27 * * *$

SIE X Perceived Social Support

$.10 *$

$-.10 *$

$-.02$

Variance Components

Intercept

$1.43 * * *$

NIE Slope

SIE Slope

.10

Note: Analyses controlled for day of week and time of day

$\mathrm{NIE}=$ negative interpersonal exchanges

SIE = supportive interpersonal exchanges

$\dagger p<.10, * p<.05, * * p<.01, * * * p<.001$ 
Daily Social Support and Drinking, 142

Table 8b: Hypothesis 4, Continued

Predictor

Solitary drinking at home

Log Odds Coefficient

Step Two:

Intercept Model

Solitary drinking at home

PSS

$-1.08 * * *$

$-.19$

Slopes Model

NIE

$-.22 * * *$

NIE X Perceived Social Support

$.12 * *$

SIE

$-.10 *$

SIE X Perceived Social Support

$-.03$

SIE $x$ NIE (interaction term)

$-.09$

SIE x NIE (interaction term) X Perceived Support $\quad-.06$

Variance Components

Intercept

$1.44 * * *$

NIE Slope

.26

SIE Slope

.11

Note: Analyses controlled for day of week and time of day

$\mathrm{NIE}=$ negative interpersonal exchanges

SIE = supportive interpersonal exchanges

$\dagger p<.10, * p<.05, * * p<.01, * * * p<.001$ 
Daily Social Support and Drinking, 143

Table 9. Hypothesis Summaries

Hypothesis

Supported/Not Supported

1

Does daily support have a direct-effect on later drinking?

2

Does daily support

Not Supported moderate the negative exchange drinking relationship?

3

Do individuals who have

Not Supported higher levels of perceived support report less drinking over the course of the study?

4

Are the moderating effects of daily support on the negative exchange drinking relationship stronger for those with higher levels of perceived social support?

Not Supported 
Daily Social Support and Drinking, 144

Figure 1. Conceptual model of where social support and drinking may influence the stress and coping process

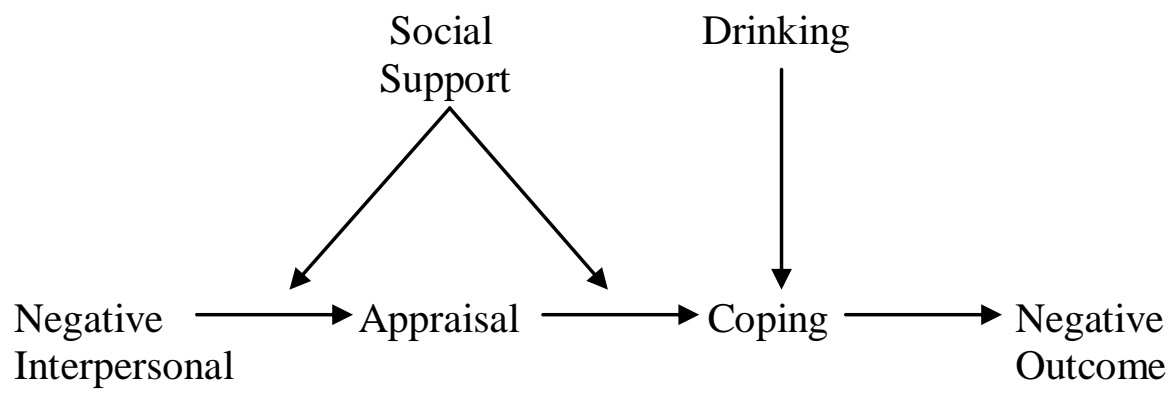


Daily Social Support and Drinking, 145

Figure 2. Two constructs of social support (perceived and received) can be examined within the direct effect and stress-buffering models.

Direct Effect Model Stress-Buffering Model

\section{Perceived}

Support

Received

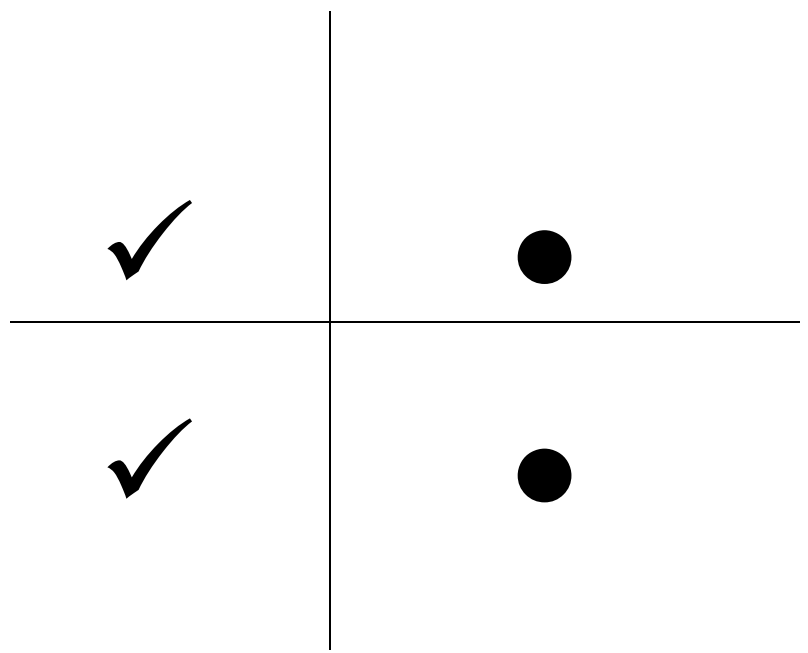

Support

The checks depict the association between perceived and received support and the direct effect model. The circles depict the association between perceived and received support and the stress-buffering model. 
Daily Social Support and Drinking, 146

Figure 3. Four Factor Model of Alcohol Consumption (Cooper, 1994)

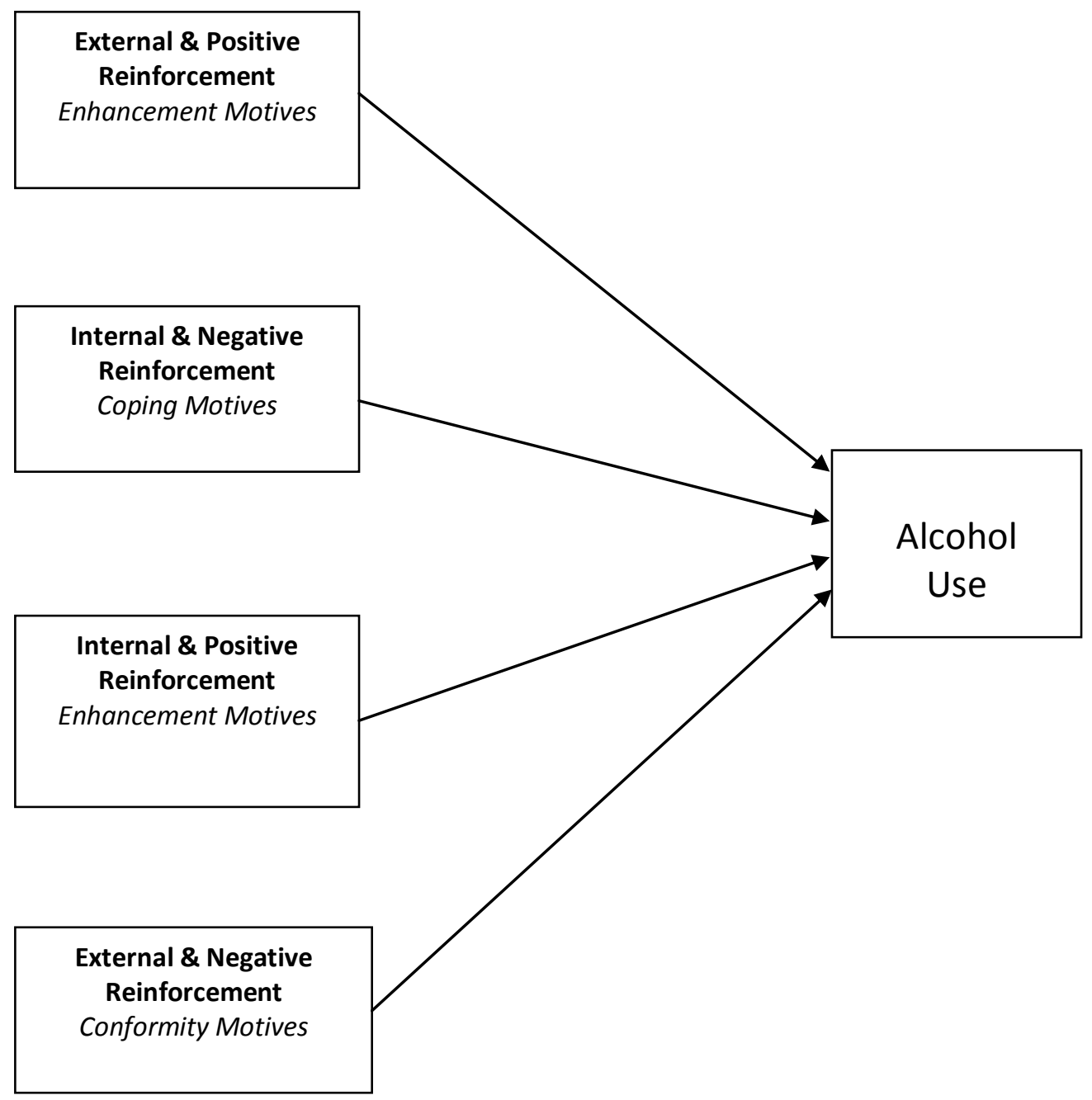


Figure 4. Daily survey schedule.

\section{Protocol}

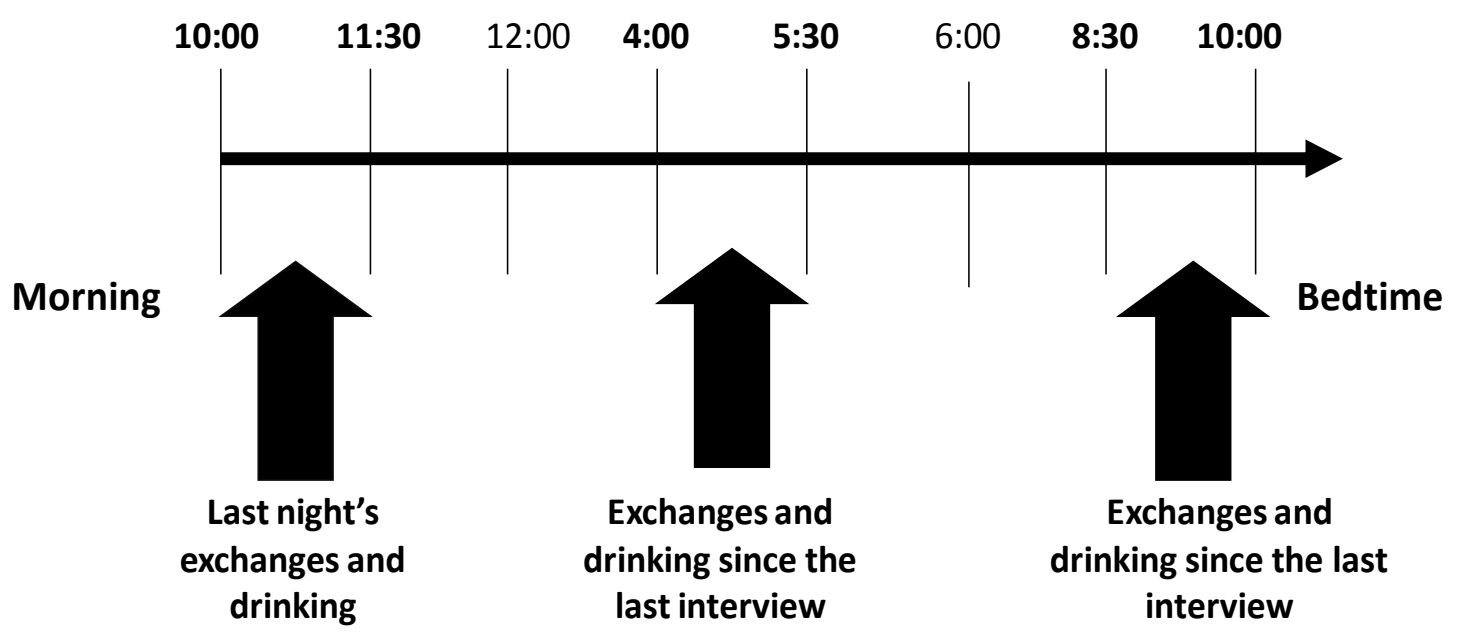

10:00 a.m. to 11:30 p.m.: Morning Survey

4:00 p.m. to 5:30 p.m.: Afternoon Survey

8:30 p.m. to 10:00 p.m.: Evening Survey 
Figure 5. Graphic depiction of Hypothesis 1 and 2.

H1: The Direct-Effect Model: At times when people report more positive socially supportive exchanges, they will report less drinking at home alone in the next time period relative to times when they report fewer positive socially supportive exchanges.

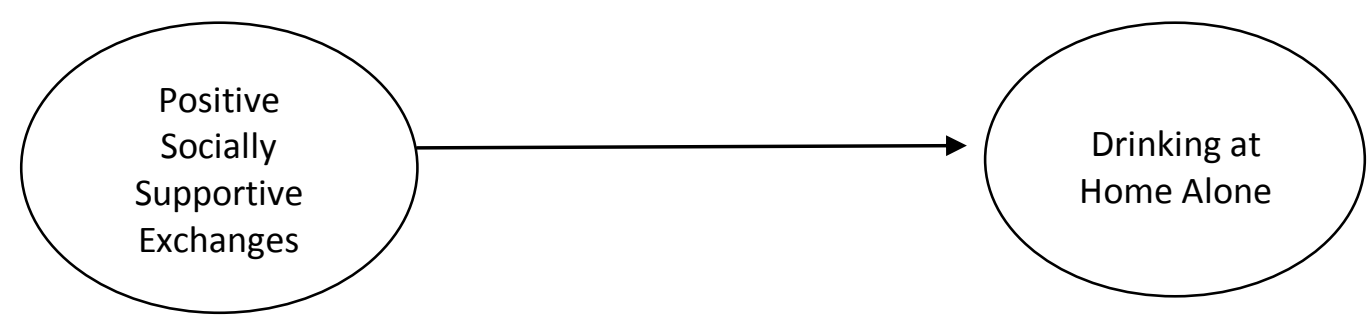

H2: The Stress-Buffering Model: Socially supportive exchanges will moderate the negative exchange-drinking relationship.

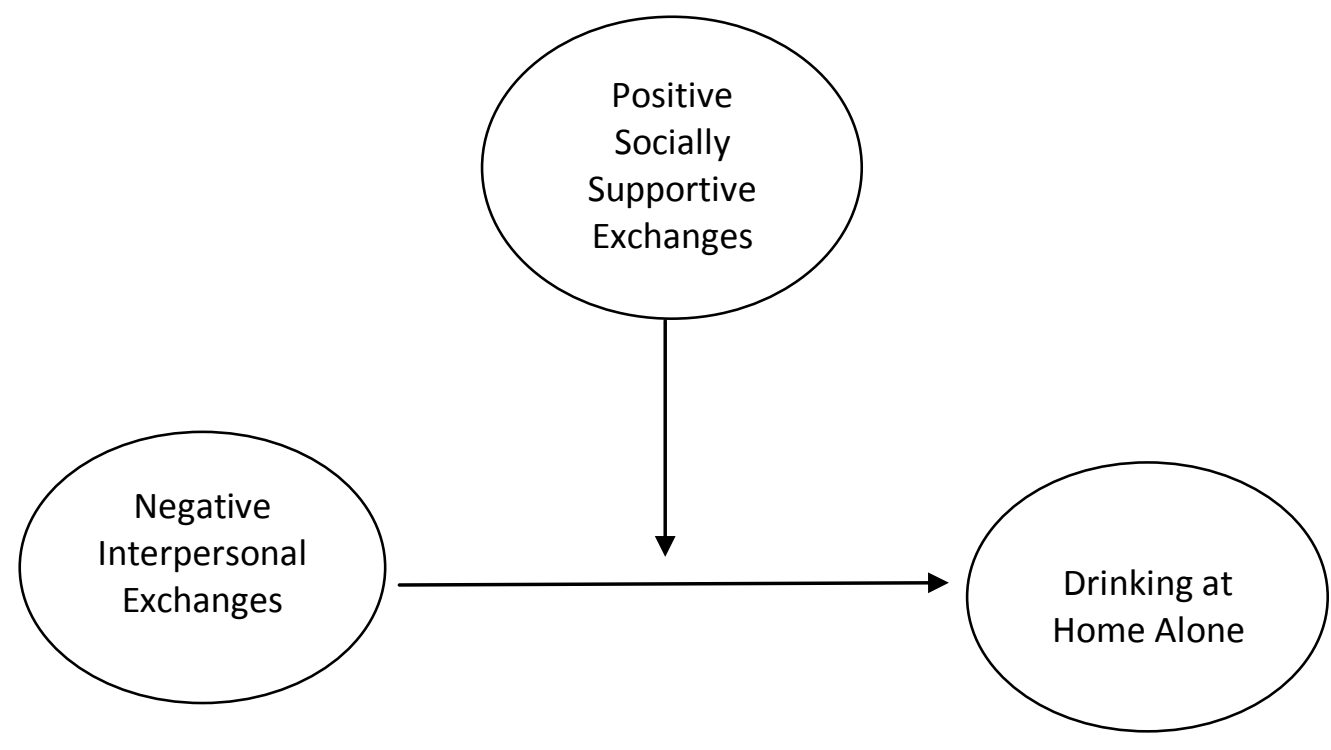


Figure 6. Graphic depiction of Hypothesis 3 and 4.

H3: Individuals with higher levels of perceived social support will report less drinking at home alone throughout the course of the study compared to those with lower perceived social support.

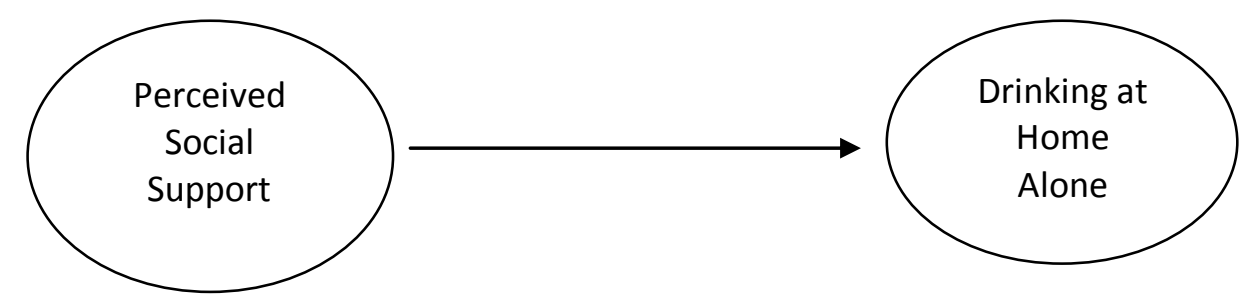

H4: The moderating effects of SIE on the NIE-subsequent solitary drinking at home relationship will be stronger for those with higher perceived social support.

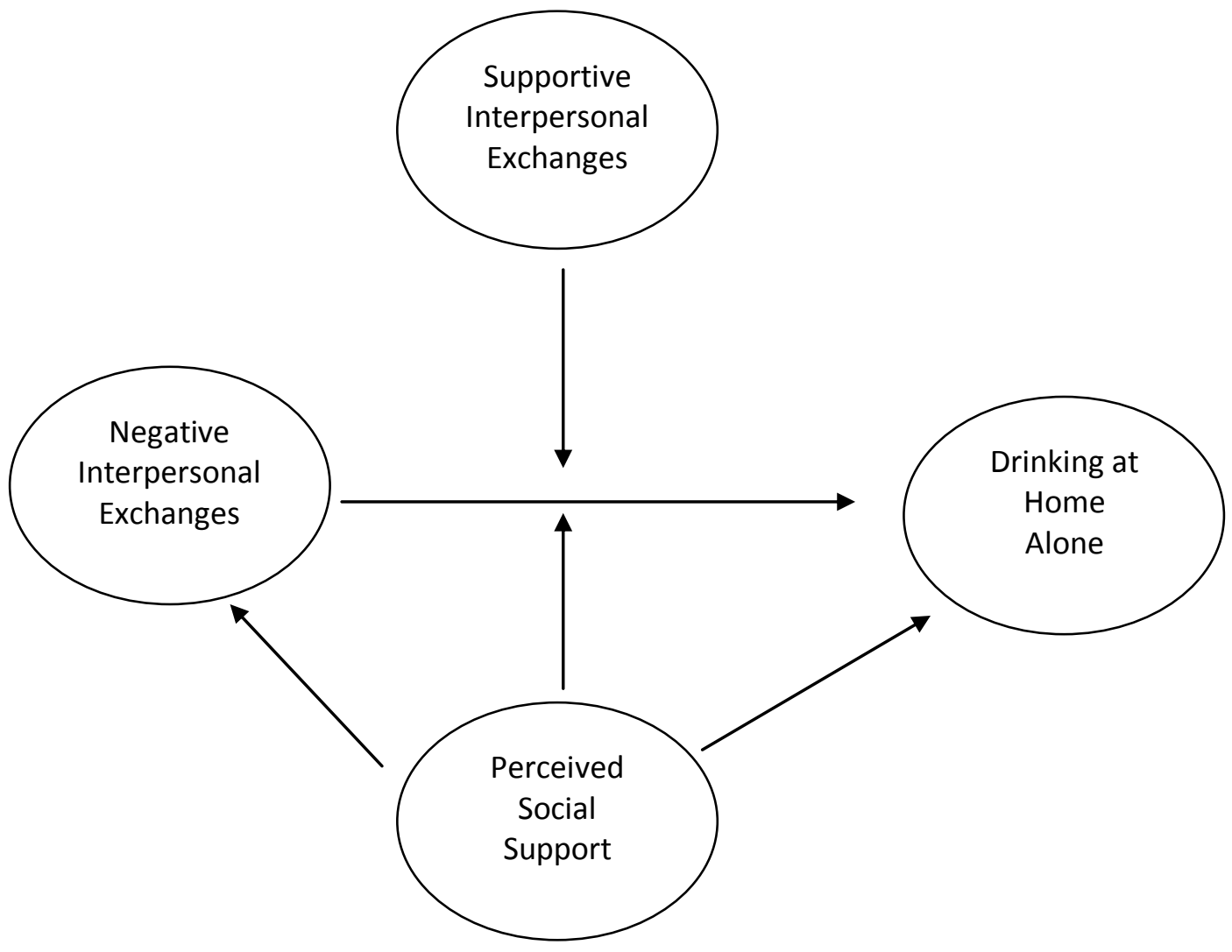


Figure 7 - Hypothesis 4, Step 1: Simple slopes for Perceived Social Support and Negative Interpersonal Exchanges

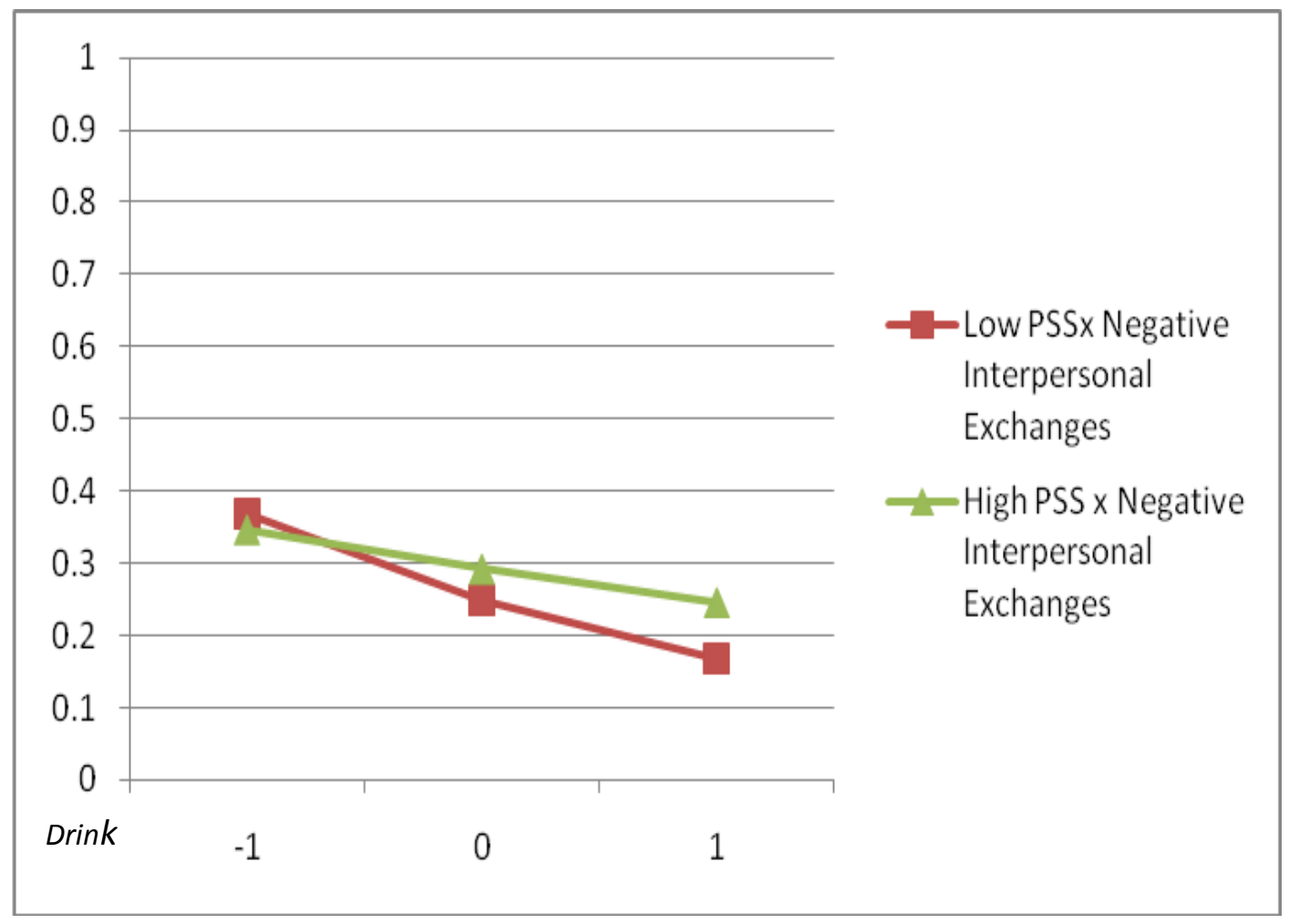


Daily Social Support and Drinking, 151

\section{References}

Affleck, G., Zautra, A., Tennen, H. \& Armeli, S. (1999). Multilevel daily process designs for consulting and clinical psychology: A preface for the perplexed. Journal of Consulting and Clinical Psychology, 67(5), 746-754.

Aiken, L. S., \& West, S. G. (1991). Multiple regression: Testing and interpreting interactions. Newbury Park, CA: Sage.

Armeli, S., Carney, M. A., Tennen, H., Affleck, G., \& O’Neil, T. (2000). Stress and alcohol use: A daily process examination of the stressor-vulnerability model. Journal of Personality and Social Psychology, 78, 979-994.

Armeli S., Todd M., \& Mohr C. (2005). A daily process approach to individual differences in stress-related alcohol use. Journal of Personality, 73(6), $1657-1686$.

Abrams, D.B., \& Niaura, R.S. (1987). Social learning theory of alcohol use and abuse. In H. Blane \& K. Leonard (Eds.), Psychological theories of drinking and alcoholism (pp. 131-178). New York: Guilford Press.

Argeriou, M. (1975). Daily alcohol consumption patterns in Boston: Some findings and a partial test of the Tuesday hypothesis. Journal of Studies on Alcohol, $36,1578-1573$.

Babbie, E. (2007). The Practice of Social Research (1 $\left.1{ }^{\text {th }} \mathrm{Ed}\right)$, Belmont, CA: Wadsworth. 
Daily Social Support and Drinking, 152

Bandura, A. (1969). Principles of Behavior Modification. New York: Holt, Rinehart \& Winston.

Baron, R.M. \& Kenny, D.A. (1986). The moderator-mediator distinction in social psychological research: Conceptual, strategic, and statistical considerations. Journal of Personality and Social Psychology, 51, 419 - 456

Barrera, M. \& Ainlay, S.L. (1983). The structure of social support: A conceptual and empirical analysis. Journal of Community Psychology, 11, 133-143.

Barrera Jr., M., Sandler, I.N., Ramsay, T.B. (1981). Preliminary development of a scale of social support: studies on college students. American Journal of Community Psychology. 9(4), 435-447.

Baumeister, R. \& Leary, M. (1995). The need to belong: Desire for interpersonal attachments as a fundamental human motivation. Psychological Bulletin, 117(3), 497-529.

Beattie M.C., Longabaugh R., Elliott G., Stout R.L., Fava J., Noel N.E. (1993). Effects of the social environment on alcohol involvement and subjective well-bring prior to alcoholism treatment. Journal of Studies on Alcohol, 54(3), 283-96.

Beck, K. H. and Treiman, K. A. (1996). The relationship of social context of drinking, perceived social norms, and parental influence to various drinking patterns of adolescents. Addictive Behaviors, 21, 633-644 
Beers, T.M. (2000). Flexible schedule and shift work: replacing the '9-to-5' workday? Monthly Labor Review, 6, 33-40.

Bell, R., LeRoy, J., \& Stephenson, J. (1982). Evaluating the mediating effects of social support upon life events and depressive symptoms. Journal of Community Psychology, 10, 325-340.

Berk, R. \& MacDonald, J.M. (2008). Overdispersion and Poisson regression. Journal of Quantitative Criminology, 24, 269-284.

Berkman, L.F. \& Syme, L. (1979). Social networks, host resistance, and mortality: A nine year follow-up study of Alameda county residence. American Journal of Epidemiology, 109, 186-203.

Berger, V.W. \& Zhang, J. (2005). Structural zeros. Encyclopedia of Statistics in Behavioral Science. Baltimore, MD: John Wiley \& Sons.

Bolger, N., Davis, A., \& Rafaeli, E. (2003). Diary methods: Capturing life as it is lived. Annual Review of Psychology, 54, 579-616.

Bolger, N., DeLongis, A., \& Kessler, R. C., \& Schilling, E. A. (1989). Effects of daily stress on negative mood. Journal of Personality and Social Psychology, 57, 808-818.

Bolger, N., \& Zuckerman, A. (1995). A framework for studying personality in the stress process. Journal of Personality and Social Psychology, 69, 890-902.

Bolger, N., Zuckerman, A., \& Kessler, R. (2000). Invisible support and adjustment to stress. Journal of Personality and Social Psychology, 79(6), 953-961. 
Bower, G. H. (1981). Mood and memory. American Psychologist, 36, 129-148.

Bowlby, J. (1969). Attachment and Loss, Volume1 Attachment. London: Hogarth Press.

Brickman, P. D., Coates, D., \& Janoff-Bulman, R. (1978). Lottery winners and accident victims: is happiness relative? Journal of Personality and Social Psychology, 36, 917-927.

Brissette, I., Cohen, S., \& Seeman, T.E. (2000). Measuring social integration and social networks. In S. Cohen, L. Underwood, \& B. Gottlieb (Eds.), Measuring and Intervening in Social Support, (pp. 53-85), New York: Oxford University Press.

Broadhead, W.E., Kaplan, B.H., James, S.A., Wagner, E.H., Schoenbach, V.J., Grimson, R., Heden, S., Tibblin, G., \& Gehlbach, S.H. (1983). The epidemiologic evidence for a relationship between social support and health. American Journal of Epidemiology, 117, 521-537.

Brodsky, A. \& Peele, S. (1999). Psychosocial benefits of moderate alcohol consumption: Alcohol's role in a broader conception of health and wellbeing. In S. Peele \& M. Grant (Eds). Alcohol and Pleasure: A Healthy Perspective (pp. 187-207). Philadelphia, PA: Brunner/Mazel.

Brown, G.W., \& Harris, T. (1978). Social origins of depression: a study of psychiatric disorders in women. Tavistock, London 
Daily Social Support and Drinking, 155

Bryk, A. S. \& Raudenbush, S. W. (1992). Hierarchical linear models: Applications and data analysis methods. Newbury Park: Sage.

Bryk, A. S., Raudenbush, S. W. \& Congdon, R. T. (1996). HLM. Hierarchical linear and nonlinear modeling with the HLM/2L and HLM/3L programs. Chicago: Scientific Software International.

Cadzow R.B. \& Servoss T.J. (2009). The association between perceived social support and health among patients at a free urban clinic. Journal of the National Medical Association, 101(3), 243-50.

Cahalan, D., Cisin, I.H. \& Crossley, H.M. (1969). American drinking practices: A national study of drinking behavior and attitudes. New Brunswick, NJ: Rutgers Center of Alcohol Studies.

Camargo, C.A., Hennekens, C.H., Gaziano, J.M., Glynn, R.J., Manson, J.E., Stampfer, M.J. (1997). Prospective study of moderate alcohol consumption and mortality in U.S. male physicians. Archives of Internal Medicine, 157, $79-85$.

Cappell, H. \& Herman, C.P. (1972). Alcohol and tension-reduction: A review. Journal of Studies on Alcohol, 33, 33-64.

Carney, M.A., Armeli, S. Tennen, H. Affleck, G., \& O'Neil, T. (2000). Positive and negative daily events, perceived stress, and alcohol use: A diary study. Journal of Consulting and Clinical Psychology, 68(5), 788-798. 
Caspi, A., Bolger, N., \& Eckenrode, J. (1987). Linking person and context in the daily stress process. Journal of Personality and Social Psychology, 52(1), 184-195.

Cassel, J. (1974). An epidemiological perspective of psychosocial factors in disease etiology. American Journal of Public Health, 64, 1040-1043.

Cassel, J. (1976). The contribution of the social environment to host resistance. American Journal of Epidemiology, 104, 107-123.

Chi, F. W., Kaskutas, L. A., Sterling, S., Campbell, C. I., \& Weisner, C. (2009). Twelve-Step affiliation and 3-year substance use outcomes among adolescents: social support and religious service attendance as potential mediators. Addiction, 104(6), 927-39.

Cobb, S. (1976). Social support as a moderator of life stress. Psychosomatic Medicine, 38(5), 300-314.

Cohen, J., Cohen, P., West, S. G. \& Aiken, L. S. (2003). Applied multiple regression/correlation analysis for the behavioral sciences. Mahwah, NJ: Lawrence Erlbaum Associates, Inc.

Cohen, S. (2001). Social relationships and susceptibility to the common cold. In C. D. Ryff \& B. H. Singer (Eds.), Emotion, Social Relationships, and Health. New York: Oxford University Press.

Cohen, S. (2004). Social relationships and health, American Psychologist, 59(8), 676-684. 
Daily Social Support and Drinking, 157

Cohen, S., Gottlieb, B., \& Underwood, L. (2000). Social relationships and health. In S. Cohen, L. Underwood, \& B. Gottlieb (Eds.). Measuring and Intervening in Social Support. New York: Oxford University Press.

Cohen, S., \& Hoberman, H.M. (1983). Positive events and social supports as buffers of life changes stress. Journal of Applied Social Psychology, 13(2), 99-125.

Cohen, S., Kaplan, J. R., \& Manuck, S. B. (1994). Social support and coronary heart disease: Underlying psychological and biologic mechanisms. In S. A. Shumaker \& S. M. Czajkowski (Eds.), Social Support and Cardiovascular Disease. New York: Plenum

Cohen, S., \& Lemay, E. (2007). Why would social networks be linked to affect and health practices? Health Psychology, 26, 410-417.

Cohen, S., \& McKay. (1984). Social support, stress, and the buffering hypothesis: A theoretical analysis. In A. Baum, J. E. Singer, \& S. E. Taylor (Eds.), Handbook of psychology and health, (pp. 253-267), Volume IV. Hillsdale, NJ: Erlbaum.

Cohen, S., \& Pressman, S. (2004). The stress-buffering hypothesis. In N. Anderson (Ed.), Encyclopedia of Health and Behavior. Thousand Oaks, CA: Sage Publications. 
Cohen S, Syme S.L. (1985). Social Support and Health. Orlando: Academic Press.

Cohen, S., \& Wills, T. A. (1985). Stress, social support and the buffering hypothesis. Psychological Bulletin, 98, 310-357.

Collins, R. L., Morsheimer, E. T., Shiffman, S., Paty, J. A., Gnys, M. \& Papandonatos, G. D. (1998). Ecological momentary assessment in a behavioral drinking moderation training program. Experimental and Clinical Psychopharmacology, 6, 306-315.

Camargo, C. A. Hennekens, R., Gaziano, I., Glynn, J.L., Manson, R. \& Stampfer, R.S. (1997). Prospective study of moderate alcohol consumption and mortality in US male physicians. Archives of Internal Medicine, 157, 79-85.

Compton, W.M., Thomas, Y.F., Stinson. F.S. \& Grant, B.F. (2007). Prevalence, correlates, disability, and comorbidity of DSM-IV drug abuse and dependence in the United States: results from the national epidemiologic survey on alcohol and related conditions. Archives of general psychiatry, 64(5), 566-76.

Conger, J. J. (1956). Alcoholism: Theory, problem and challenge. II. Reinforcement theory and the dynamics of alcoholism, Journal of Studies on Alcohol, 13, 296-305.

Cook, T.D., \& Campbell, D.T. (1979). Quasi-Experimentation: Design and Analysis Issues for Field Settings. Chicago: Rand McNally. 
Daily Social Support and Drinking, 159

Cooper, M. L. (1994). Motivations for alcohol use among adolescents: Development and validation of a four-factor model. Psychological Assessment, 6(2), 117128.

Cooper, L., Frone, M., Russell, M., \& Mudar, P. (1995). Drinking to regulate positive and negative emotions: A motivational model of alcohol use. Journal of Personality and Social Psychology, 69(5), 990-1005.

Cooper, M. L., Russell, M., Skinner, J. B., Frone, M. R., \& Mudar, P. (1992a). Stress and alcohol use: Moderating effects of gender, coping and alcohol expectancies. Journal of Abnormal Psychology, 101, 139-152.

Cooper, M.L., Russell, M., Skinner, J.B., \& Windle, M. (1992b). Development and validation of a three-dimensional measure of drinking motives. Psychological Assessment, 4, 123-132.

Cooper, M.L., Russell, M., \& George, W.H. (1988). Coping, expectancies, and alcohol abuse: A test of social learning formulations. Journal of Abnormal Psychology, 97, 218-230.

Coriell, M., \& Cohen, S. (1995). Concordance in the face of a stressful event: When do members of a dyad agree that one person supported the other? Journal of Personality and Social Psychology, 69, 289-299.

Cox, W. M., \& Klinger, E. (1988). A motivational model for alcohol use. Journal of Abnormal Psychology, 97(2), 168-180. 
Daily Social Support and Drinking, 160

Cox, M., \& Klinger, E. (1990). Incentive motivation, affective change, and alcohol use: A model. In M. Cox (Ed.), Why people drink (pp. 291-311), New York: Gardner Press.

Cox, M., \& Klinger, E. (1990). Incentive motivation, affective change, and alcohol use: A model. In M. Cox (Ed.), Why people drink (pp. 291-311), New York: Gardner Press.

Coyne, J. C., DeLongis, A. (1986). Going beyond social support: The role of social relationships in adaptation. Journal of Consulting and Clinical Psychology, 54,454

Coyne, J., Wortman, C.B., \& Lehman, D. (1988). The other side of support: Emotional overinvolvement and miscarried helping. In B. Gottlieb (Ed). Marshaling social support: Formats, processes, and effects. (pp. 305-330). Thousand Oaks, CA, US: Sage Publications, Inc.

Cutrona, C. (1986). Behavioral manifestations of social support: A microanalytic investigation. Journal of Personality and Social Psychology, 51(1), 201208.

Cutrona, C. (1990). Stress and social support: In search of optimal matching. Social \& Clinical Psychology, 9(1), 3-14.

Curran, P. J., Bauer, D. J, \& Willoughby, M. T. (2004). Testing main effects and interactions in hierarchical linear growth models. Psychological Methods, 9, 220-237. 
Daily Social Support and Drinking, 161

Cutter, H.S., \& O'Farrell, T.J. (1984). Relationship between reasons for drinking and customary drinking behavior. Journal of Studies on Alcohol, 45(4), $321-5$.

Davidowitz, M., \&Myrick, R.D. (1984). Responding to bereaved: An analysis of "helping" statement. Research Record, 1, 35-42.

Deci, E. L., \& Ryan, R. M. (2000). The "what" and "why" of goal pursuits: Human needs and the self-determination of behavior. Psychological Inquiry, 11, 227-268.

Del Boca, F.K. Darkes, J. (2003). The validity of self-reports of alcohol consumption: state of the science and challenges for research. Addition, 98, $1-12$.

DeLongis, A., Capreol, M. J., Holtzman, S., O'Brien, T. B., \& Campbell, J. (2004). Social support and social strain among husbands and wives: A multilevel analysis, Journal of Family Psychology, 18, 470-479.

DeLongis, A., Coyne, J.C., Dakof, G., Folkman, S., \& Lazarus, R.S. (1982).

Relationship of daily hassles, uplifts, and major life events to health status. Health Psychology, 1(2), 119-136

DeLongis, A., Folkman, S., \& Lazarus, R.S. (1988). The impact of daily stress on health and mood: Psychological and social resources as mediators. Journal of Personality and Social Psychology, 54(3), 486-495. 
Daily Social Support and Drinking, 162

Diener, E. (1984). Subjective well-being. Psychological Bulletin, 95(3), 542-575.

Diener, E. \& Diener, C. (1996). Most people are happy. Psychological Science, 7,181-185.

Diener, E., Lucas, R. E., \& Scollon, C. (2006). Beyond the hedonic treadmill: revising the adaptation theory of well-being. American Psychologist, 61, $305-314$.

Dillman, D.A., Lesser, V., Mason, R., Carlson, J., Willits, F., Robertson, R., \& Burke, B. (2007). Personalization of mail surveys for general public and populations with a group identity: Results from nine studies. Rural Sociology 72(4), 632-646.

Dunkel-Schetter, C. (1984). Social support and cancer: Findings based on patient interviews and their implications. Journal of Social Issues, 40(4), 77-98.

Dunkel-Schetter, C., Blasband, D., Feinstein, L. G., \& Bennett Herbert, T. (1992). Elements of supportive interactions: When are attempts to help effective? In S. Spacapan \& S. Oskamp (Eds.) Helping and being helped in the real world (pp. 83-114). Newbury Park, CA: Sage Publications.

Dunkel-Schetter, C., Sagrestano, L., Feldman, P \& Killingsworth, C. (1996). Social support and pregnancy: A comprehensive review focusing on ethnicity and culture. In G. Pierce, B. Sarason, \& I. Sarason (Eds.), Handbook of Social support and the family. (pp. 375-412) New York: Plenum. 
Durkheim, E. (1951). Suicide, A Study in Sociology. Ornstein, R. \& Swencionis, C. (Eds). New York: Free Press.

Eaton, W.W. (1978). Life events, social supports, and psychiatric symptoms: A reanalysis of the New Haven data. Journal of Health and Social Behavior, $19,230-234$.

Eckenrode, J. \& Hamilton, S. (2000). Social relationships and health. In S. Cohen, L. Underwood, \& B. Gottlieb (Eds.), Measuring and intervening in social support. (pp. 246-271) New York: Oxford University Press.

Enders, C.K. \& Tofighi, D. (2007). Centering predictor variables in cross-sectional multilevel models: A new look at an old issue. Psychological Methods, 12, $121-138$

Erikson, E. (1959). Identity and the life cycle, Psychological Issues, 1, 18-164.

Farber, P.D., Khavari, K.A., Douglass, F.M. A factor analytic study of reasons for drinking: Empirical validation of positive and negative reinforcement dimensions.

Feldman, S., Downey, G., \& Schaffer-Neitz. (1999). Pain, negative mood, and perceived support in chronic pain patients: A daily diary study of people with reflex sympathetic dystrophy syndrome. Journal of Consulting and Clinical Psychology, 61(5), 776-785. 
Daily Social Support and Drinking, 164

Fredrickson, B. (2001). The role of positive emotions in positive psychology: The broaden-and-build theory of positive emotions. American Psychologist, 56(3), 218-226.

Fredrickson, B. \& Joiner, T. (2002). Positive emotions trigger upward spirals toward emotional well-being. Psychological Science, 13(2), 172-176.

Gable, S., Reis, H., Impett, E., \& Asher, E. (2004). What do you do when things go right? The intrapersonal and interpersonal benefits of sharing positive events, Journal of Personality and Social Psychology, 87(2), 228-245.

Gardner, W., Mulvey, E.P., \& Shaw, E.C. (1995). Regression analyses of counts and rates: Poisson, overdispersed Poison, and negative binomial models. Psychological Bulletin, 118, 392-404.

Gellar, P.A., Graf, M.C., Dyson-Washington, F. (2003). Women's health psychology. In Weiner, I., Freeheim, D., Nezu, A., Nezu, C., \& Geller P. (Eds). Handbook of Psychology: Health Psychology, 518-545.

Gleason, M.E.J., Iida, M., Bolger, N., \& Shrout, P.E. (2003). Daily supportive equity in close relationships. Personality and Social Psychology Bulletin, 29(8), 1036-1045.

Goldman, M. S., Del Boca, F. K., \& Darkes, J. (1999). Alcohol expectancy theory: The application of cognitive neuroscience. In K. Leonard \& H. Blane (Eds.), Psychological theories of drinking and alcoholism (pp. 203-246). New York: Guilford Press. 
Daily Social Support and Drinking, 165

Gottlieb, B.H. (1984). Social support and the study of personal relationships. Journal of Social and Personal Relationships, 2, 351-375.

Greeley, J. and Oei, T. (1999) Alcohol and tension reduction. In Psychological Theories of Drinking and Alcoholism, Leonard, K. E. and Blane, H. T. eds, pp. 14-53. The Guilford Press, New York.

Groh, D.R., Jason, L.A., Davis, M.I., Olson, B.D., \& Ferrari, J.R. (2007). Friends, family, and alcohol abuse: An examination of general and alcohol-specific social support. American Journal on Addition, 16, 49-55.

Handley E.D. \& Chassin L. (2008). Stress-induced drinking in parents of adolescents with externalizing symptomatology: the moderating role of parent social support. American Academy of Psychiatrists in Alcoholism and Addictions, 17(6), 469-77.

Headey, B. (2008). The set-point theory of well-being: Negative results and consequent revisions. Social Indicators Research, 85, 389-403.

Helgeson, V. S., Cohen, S., Schulz, R., Yasko, J. (2001). Long-term effects of educational and peer discussion group support interventions on adjustment to breast cancer. Health Psychology, 20, 387-392.

Helgeson, V. S., \& Gottlieb, B. H. (2000). Support groups. In S. Cohen, L. G. Underwood, \& B. H. Gottlieb (Eds.), Social support measurement and intervention: A guide for health and social scientists. New York: Oxford University Press 
Daily Social Support and Drinking, 166

Heller, K., Swindle Jr., R.W., \& Dusenbury, L. (1986). Component social support processes: Comments and integration. Journal of consulting and Clinical Psychology, 54(4), 466-470.

Hesselbrock, M. N., Meyer, M. N., \& Keener, J. J. (1985). Psychopathology in hospitalized alcoholics. Archives of General Psychiatry, 42, 1050-1055.

Higgins-Biddle, J.C. and Babor, T.F., (1996). Reducing risky drinking via screening and brief intervention, "Alcoholism \& Drug Abuse Weekly, 8, 5- 21.

Higgins R. L., Marlatt G. A. (1973). Fear of interpersonal evaluation as a determinant of alcohol consumption in male social drinkers. Journal of Abnormal Psychology, 84, 644-651.

Hirsch, B.J. (1980). Natural support systems and coping with major life changes. American Journal of community Psychology, 8, 159-172.

Hofer, S. M., \& Hoffman, L. (2007). Statistical analysis with incomplete data in the context of the ecological model. In T. D. Little, J. A. Bovaird, \& N. A. Card (Eds.), Modeling Contextual Effects in Longitudinal Studies (pp. 13-32). Mahwah, NJ: Erlbaum

Hofman, D. (1998). Centering decisions in hierarchical linear models: Implications for research in organizations. Journal of Management, 24, 623-641.

Hofman, D., \& Gavin, M. (1998). Centering decisions in hierarchical linear models: Theoretical and methodological implications for organizational science. Journal of Management, 24, 623-641. 
Daily Social Support and Drinking, 167

House, J.W., Umberson, D., \& Landis, K.R. (1988). Structures and processes of social support. Annual Review of Sociology, 14, 293-318.

Howell, D.C. (2002). Statistical methods for psychology, $5^{\text {th }}$ ed., Pacific Grove, CA: Wadsworth.

Hox, J. (2002). Multilevel Analysis; Techniques and Applications. Mahwah, New Jersey.

Hufford, M. R., Shields, A. L., Shiffman, S., Paty, J., \& Balabanis, M. (2002). Reactivity to ecological momentary assessment: An example using undergraduate problem drinkers. Psychology of Addictive Behaviors, 16, $205-211$

Hull, J. G. (1981) A self-awareness model of the causes and effects of alcohol consumption. Journal of Abnormal Psychology, 90, 586-600.

Hull, J. G., Levenson, R.W., Young, R.D., \& Sher, K. J. (1983). Self-awarenessreducing effects of alcohol consumption. Journal of Personality and Social Psychology, 44, 461-473.

Hull, J. G., \& Levy, A. S. (1979). The organizational functions of the self: An alternative to the Duval and Wicklund model of self-awareness. Journal of Personality and Social Psychology, 37, 756-768.

Humphreys, K., Moos, R. H., \& Finney, J. W. (1995). Two pathways out of drinking problems. Addictive Behaviors, 20, 427-441.

Hussong, A. M. (2007). Predictors of drinking immediacy following daily sadness: 
Daily Social Support and Drinking, 168

An application of survival analysis to experience sampling data. Addictive Behaviors, 32, 1054-1065.

Hussong, A.M., Galloway, C.A., \& Feagans, L.A. (2005). Coping motives as a moderator of daily mood-drinking covariation Journal of Studies on Alcohol, 66, 344-53.

Ikehara, S., Iso, H., Yamagishi, K., Yamamoto, S., Inoue, M., \& Tsugane, S. (2009). Alcohol consumption, social support and risk of stroke and coronary heart disease among Japanese men: The JPHC study. Alcoholism: Clinical \& Experimental Research, 33, 1025-1032.

Judd, C.M. \& Kenny, D.A. (1981). New York, NY: Cambridge University Press. Kahneman, D. \& Krueger, A.(2006). Developments in the measurement of subjective well-being." Journal of Economic Perspectives 20, 3-24.

Kawachi, I. \& Berman, L.F. (2001). Social ties and mental health. Journal of Urban Health, 78, 458-467

Kenny, D. A., Bolger, N., \& Kashy, D. A. (1999). Traditional methods for estimating multilevel models. In D. S. Moskowitz \& S. Hershberger (Eds.), Modeling intraindividual variability with repeated measures data: Method and applications. Englewood Cliffs, NJ: Erlbaum.

Kessler, R.C., \&McLeod, J.D. (1985). Social support and mental health in community samples. In S. Cohen \& S. Lyme (Eds), Social Support and Health, (pp. 219-240), Orlando: Academic Press. 
Koniak-Griffln, D., Lominska, S., \& Brecht, M. L. (1993). Social support during adolescent pregnancy: A comparison of three ethnic groups. Journal of Adolescence, 16, 43-56.

Kranzler, H.R., Armeli, S., Feinn, R., \& Tennen, H. (2004). Targeted Naltrexone Treatment Moderates the Relations Between Mood and Drinking Behavior Among Problem Drinkers. Journal of Consulting and Clinical Psychology, 72, 317-327.

Kreft, I., \& De Leeuw, J. (1998). Introducing multilevel modeling. New York: Sage.

Kreft, I., De Leeuw, J., \& Aiken, L. S. (1995). The effects of different forms of centering in hierarchical linear models. Multivariate Behavioral Research, $30(1), 1-21$.

La Guardia, J. G., \& Patrick, H. (2008). Self-determination theory as a fundamental theory of close relationships. Canadian Psychology, 49, 201-209.

Lakey, B. \& Cohen, S. (2000). Social relationships and health. In S. Cohen, L. Underwood, \& B. Gottlieb (Eds.), Measuring and intervening in social support. (pp. 29-52), New York: Oxford University Press.

Larson, R. \& Csikszentmihalyi, M. (1983). The experience sampling method. In H.T. Reis (Ed.), Naturalistic Approaches to Studying Social Interaction. San Francisco: Jossey-Bass. 
Daily Social Support and Drinking, 170

Lazarus, R.S., \& Folkman, S. (1987). Transactional theory and research on emotions and coping. European Journal of Personality, 1, 141-169.

Lazarus, R.S., Lazarus, B., Campus, J.J., Tennen, R., \& Tennen, H. (2006). Emotions and interpersonal relationships: Toward a person-centered conceptualization of emotions and coping, Journal of Personality, 74, $9-46$.

Lepore, S. J., Silver, R.C., Wortman, C.B., \& Wayment, H. A. (1996). Social constraints, intrusive thoughts, and depressive symptoms among bereaved mothers. Journal of Personality and Social Psychology.70(2), 271-282

Litt, M. D., Cooney, N. L., \& Morse, P. (1998). Ecological momentary assessment (EMA) with treated alcoholics: Methodological problems and potential solutions. Health Psychology, 17, 48-52.

Little, R., \& Rubin, D. (1987). Statistical analysis with missing data. New York: John Wiley.

Marco, C. A., Neale, J.M., Swartz, J.E., Shiffman, S., \& Stone, A.A. (1999). Coping with daily events and short-term mood changes: An unexpected failure to observe effects of coping. Journal of Consulting and Clinical Psychology, 67, 755-764.

Markus, H., \& Kitayama, S. (1991). Culture and the self: Implications for cognition, emotion, and motivation. Psychological Review, 98, 224-253. 
Marlatt, G. A., Kosturn, C. F., \& Lang, A. R. (1975). Provocation to anger and opportunity for retaliation as determinants of alcohol consumption in social drinkers. Journal of Abnormal Psychology. 84, 652-659.

Martire, L., Parris Stephens, M.A., Druley, J., \& Wojno. (2002). Negative reactions to received spousal care: Predictors and consequences of miscarried support. Health Psychology, 21(2), 167-176.

Maisto, S. A., Carey, K. B., \& Bradizza, C. M. (1999). Social learning theory. In K. E. Leonard \& H. T. Blane, (Eds.), Psychological theories of drinking and alcoholism (2nd ed., pp. 107-163). New York: Guilford Press.

Mertler, C. \& Vannatta, R.A. (2005). Advanced and Multivariate Statistical Methods. (pp. 31-32). Pyrczak Publishing; California.

Miller, P.M. \& Ingham, J.G. (1976). Friends, confidants, and symptoms. Social Psychiatry, 11, 51-58.

Miller, J.M. \& Miller, M. D. (2008). No zero left behind: Comparing the fit for zeroinflation models as a function of skew and proportion of zeros. Interstat, 11 .

Mohr, C.D., Brannan, D., Wendt, S., Jacobs, L. \& Wang, M. (Under-review). Within-person indicators of health, Psychology Science.

Mohr, C.D. (2007). A daily process study of motivational models of alcohol consumption (or study of adult daily health). Final Progress Report: 5R03AA14598-2 
Daily Social Support and Drinking, 172

Mohr, C. D., Armeli, S., Tennen, H., Carney, M. A., Affleck, G., \& Hromi, A. (2001). Daily interpersonal experiences, context and alcohol consumption: Crying in your beer and toasting good times? Journal of Personality and Social Psychology, 80, 489-500.

Mohr, C. D., Armeli, S., Tennen, H., \& Todd, M. (2009). The complexities of modeling mood-drinking relationships: Lessons learned from daily process research. Kassel, J.D. (Ed). Substance abuse and emotion. (pp. 189-216). Washington, DC, US.

Mohr, C.D. \& Brannan, D. (unpublished manuscript). Positive and negative interpersonal exchange in an adult sample.

Mulford, H.A., \& Miller, D.E. (1963). Preoccupation with alcohol and definitions of alcohol: A replication study of two cumulative scales. Quarterly, Journal of Studies on Alcohol, 24, 682-696.

Mulia, N., Schmidt, L., Bond, J., Jacobs, L., \& Korcha, R. (2008). Stress, social support and problem drinking among women in poverty. Addiction, 103(8), 1283-1293.

Mulia N, Ye Y, Zemore S, Greenfield T. (2008). Social disadvantage, stress, and alcohol use among Black, Hispanic, and White Americans: Findings from the 2005 U.S. National Alcohol Survey. Journal of Studies on Alcohol Drugs, 69, 824-833 
Daily Social Support and Drinking, 173

Murphy, K., \& Myors, B. (2004). Statistical power analysis: A simple and general model for traditional and modern hypothesis tests 2nd ed. Erlbaumm.

Newsom, J., Nishishiba, M., Morgan, D., \& Rook, K. (2003) The relative importance of three domains of positive and negative social exchanges: A longitudinal model with comparable measures. Psychology and Aging, $18(4), 746-754$

Norbeck, J. S., \& Anderson, N. J. (1989). Psychosocial predictors of pregnancy outcomes in low-income Black, Hispanic, and White women. Nursing Research, 38, 204-209.

Park C.L., Armeli S., \& Tennen H. (2004). Appraisal-coping goodness of fit: a daily internet study. Personality and Social Psychology Bulletin, 30(5), 558-69.

Pierce, R. S., Frone, M. R., Russell, M., \& Cooper, M. L. (1996). Relationship of financial strain and psychosocial resources to alcohol use and abuse: The mediating role of negative affect and drinking motives. Journal of Health and Social Behavior, 35, 291-308.

Perrine, M.W., Mundt, J.C., Searles, J.S. \& Lester, L. S. (1995). Validation of daily self-report alcohol consumption using interaction voice response (IVR) technology. Journal of Studies on Alcohol, 56, 487-490. 
Daily Social Support and Drinking, 174

Pierce, G.R., Sarason, B.R., Sarason, I.G., Joseph, H.J., \& Henderson, C.A. (1996). Conceptualizing and assessing social support in the context of the family. In G.R. Pierce, B.R. Sarason, and I.G. Sarason (Eds.), The handbook of social support and the family (pp. 3-23). New York: Plenum.

Pennebaker \& Chung, J.W., \& Chung, C.K. (2007). Expressive writing, emotional upheavals, and health. In H. Friedman and R. Silver (Eds.), Foundations of Health Psychology (pp. 263-284). New York: Oxford University Press

Preacher, K. J. (2003). A primer on interaction effects in multiple linear regression. Retrieved from: http://www.people.ku.edu/ preacher/interact/nteractions.htm

Preacher, K. J., Curran, P. J., \& Bauer, D. J. (2006). Computational tools for probing interaction effects in multiple linear regression, multilevel modeling, and latent curve analysis. Journal of Educational and Behavioral Statistics, $31,437-448$

Procidano, M.E. \& Heller, K. (1983). Measures of perceived social support from friends and from family: Three validation studies. American Journal of Community Psychology, 11(1), 1-24.

Ptacek, J.T., Smith, R.E., Espe, K., \& Rafferty, B. (1994). Limited correspondence between daily coping reports and retrospective coping recall. Psychological Assessment, 6,41-49. 
Daily Social Support and Drinking, 175

Raudenbush, S.W., Bryk, A.S., Cheong, Y.F., \& Congdon, R.T. (2000). HLM5.

Hierarchical Linear and Nonlinear Modeling. Chicago: Scientific Software International.

Read, J.P., Wood, M.D., Kahler, C.W., Maddock, J.E., Palfai, T. (2003).

Examining the role of drinking motives in college student alcohol use and problems. Psychology of Addictive Behaviors, 17, 13-23.

Reinhardt, J.P., Boerner, K., \& Horowitz, A. (2006). Good to have but not to use: Differential impact of perceived and received support on well-being. Journal of Social and Personal Relationships, 23(1), 117-129.

Reis, H., \& Gable, S., (2000). Event-sampling and other methods for studying everyday experience. In H.T. Reis \& C.M. Judd (Eds). Handbook of Research Methods in Social and Personality Psychology. Cambridge, UK: Cambridge University Press.

Reis, H.T., Smith, S.M., Carmichael, C.L., Caprariello P.A., Tsai, F.F., Rodrigues, A., \& Maniaci, M.R. (2010). Are you happy for me? How sharing positive events with others provides personal and interpersonal benefits. Journal of Personality and Social Psychology, 2, 311-329.

Repetti, R. L. (1992). Social withdrawal as a short-term coping response to daily stressors. In H.S. Friedman (Ed.), Hostility, coping, and health. (pp. 151165). Washington DC: American Psychological Association. 
Ruehlman, L. \& Karoly, P. (1991). With a little flak from my friends: Development and preliminary validation of the Test of Negative Social Exchange (TENSE). Psychological Assessment, 3, 97-104.

Robins L.N., Cottler, L., Buckholz ,K., \& Compton ,W. (1998). The Diagnostic Interview Schedule for DSM-IV. Washington University School of Medicine; St. Louis, MO.

Robinson, M.D., \& Clore, G.L. (2002). Belief and feeling: Evidence for an accessibility model of emotional self-report. Psychological Bulletin, 128, 934-960.

Rodgers, B. Korten, A.E., Jorm, A.F., Christensen, H., Henderson, S. \& Jacomb, P.A. (2000). Risk factors for depression and anxiety in abstainers, moderate drinkers and heavy drinkers. Addiction, 95(12), 1833-1845.

Rodriguez, M.S. \& Cohen, S. (1998). Social support. Encyclopedia of Mental Health, 3, $535-544$.

Rook, K. (1984). The negative side of social interaction: Impact on psychological well-being. Journal of Personality and Social Psychology, 46(5), 10971108.

Rook, .S. \& Pietromonaco, P. (1987). Close relationships: Ties that heal or ties that bind? Advances in Personal Relationships, 1, 1-35. 
Ryff, C. D. (1989). Happiness is everything, or is it? Explorations on the meaning of psychological well-being. Journal of Personality and Social Psychology, 57(6), 1069-1081.

Ryff, C.D. and Heidrich, S.M. (1997). Life experiences and well-being: Explorations on what happens and how it matters. International Journal of Behavioral Development, 20, 193-206.

Salant, P., \& Dillman, D. A. (1994). How to Conduct Your Own Survey. New York: John Wiley and Sons.

Sarason, I.G., Sarason, B.R., \& Shearin, E.N. (1986). Social support as an individual difference variable: Its stability, origins, and relational aspects. Journal of Personality and Social Psychology, 50, 845-855.

Sarason, B., Sarason, I., \& Gurung, R. (2001).Close personal relationships and health outcomes: A key to the role of social support. Duck, S. (Ed). Handbook of personal relationships: Theory, research and interventions, 2nd ed., (pp. 547-573). Hoboken, NJ, US: John Wiley \& Sons, Inc.

Sarason, B. R., Sarason, I.G., \& Pierce, G.R. (1990). Social support: The sense of acceptance and the role of relationships. In B.R. Sarason, I.G. Sarason, \& G.R. Pierce, (Eds). Social support: An interactional view. (pp. 9-25). Oxford, England: John Wiley \& Sons.

Sarason, I.G., Sarason, B.R., Potter, B.R., \& Antoni, M.H. (1985). Life events, social support, and illness, Psychosomatic Medicine, 47, 156-163. 
Saunders, J.B. (1998). Defining the beneficial patterns of alcohol consumption: A survey of clinicians. Prepared for International Center for Alcohol Policies, August, 19, 1998. (http://www.icap.org.publications/beneficial_patterns.html).

Sayette, M.A. (1993). An appraisal-disruption model of alcohol's effects on stress responses in social drinkers. Psychological Bulletin, 114, 459-476.

Sayette, M.A. (1999). Does drinking reduce stress? Alcohol Research \& Health, 24, 250-255.

Scherbaum, C., \& Ferreter, J. (2009). Estimating statistical power and required sample sizes for organizational research using multilevel modeling. Organizational Research Methods, 12, 347-367.

Schuster, T.L., Kessler, R.C., Aseltine, R.H. Jr. (1990). Positive interactions, negative interactions, and depressed mood. American Journal of Community Psychology, 18, 423-438.

Sher, K. J. (1987). Stress response dampening. In H. T. Blane \& K. E. Leonard (Eds.), Psychological theories of drinking and alcoholism (pp. 227-271). New York: Guilford Press.

Shumaker, S. A., \& Brownell, A. (1984). Toward a theory of social support: Closing conceptual gaps. Journal of Social Issues, 40(4), 11-36.

Smyth, J.M. \& Stone, A.A. (2003). Ecological momentary assessment research in behavioral medicine. Journal of Happiness, 4, 35-52. 
Daily Social Support and Drinking, 179

Snijders, T. A. B., \& Bosker, R. J. (1999). Multilevel analysis: An introduction to basic and advanced multilevel modeling. London, UK: Sage Publications.

Snyder, M., White, P. (1982). Moods and memories: Elation, depression, and the remembering of the events of one's life. Journal of Personality, 50, 149-167.

Sobell, L. C., \& Sobell, M. B. (1992). Timeline follow-back: A technique for assessing self-reported alcohol consumption. In R. Z. Litten, \& J. P. Allen (Eds.), Measuring Alcohol Consumption: Psychosocial and Biochemical Methods (pp. 41-72). Totowa, NJ: Humana Press.

Spector, P.E., \& Jex, S.M. (1998). Development of four self-report measures of job stressors and strain: Interpersonal Conflict at Work Scale, Organizational Constraints Scale, Quantitative Workload Inventory, and Physical Symptoms Inventory. Journal of Occupational Health Psychology, 3, 356-367.

Steele, C. M., \& Josephs, R. A. (1990). Drinking your troubles away II: An attention -allocation model of alcohol's effect on psychological stress. Journal of Abnormal Psychology, 97, 196-205.

Stone, A., Schwartz, J., Schwarz, N., Schkade, D., Krueger, A. \& Kahneman, D. (2006). A population approach to the study of emotion: Diurnal rhythms of a working day examined with the Day Reconstruction Method (DRM). Emotion, 6, 139-149. 
Daily Social Support and Drinking, 180

Stroebe, W., \& Stroebe, M. (1996). The social psychology of social support. In Social psychology: Handbook of basic principles. Guilford Publications, Inc.

Swendsen, J. D., Tennen, H., Carney, M. A., Affleck, G., Willard, A., \& Hromi, A. (2000). Mood and alcohol consumption: An experience sampling test of the self-medication hypothesis. Journal of Abnormal Psychology, 109, 198-204.

Tabachnick, B.G. \& Fidell, L.S. (1996). Using Multivariate Statistics ( $3^{\text {rd }}$ ed). New York: HarperCollins.

Tennen, H., \& Affleck, G. (1996). Daily processes in coping with chronic pain: Methods and analytic strategies. In M. Zeidner \& N. Endler (Eds.), Handbook of coping: Theory, research, applications (pp. 151-177). New York: Wiley.

Tennen, H., Affleck, G., Armeli, S., \& Carney, M.A. (2000). A daily process approach to coping: Linking theory, research and practice. American Psychologist, 55, 626-636.

Tennen, H., Affleck, G., Coyne, J.C., Larsen, R.J., \& DeLongis, A. (2006). Paper and plastic in daily diary research: Comment on Green, Rafaeli, Bolger, Shrout, and Reis. Psychological Methods, 11(1),112-118.

Thayer, R.E. (1989). The biopsychology of mood and arousal. New York: Oxford University Press. 
Daily Social Support and Drinking, 181

Thibaut, J. W.; Kelley, H. H. (1959). The social psychology of groups. New York: Wiley.

Thoits, P.A. (1982). Life stress, social support, and psychological vulnerability: Epidemiological considerations. Journal of Community Psychology, 10(4), $341-362$

Thoits, P.A. (1986). Social support as coping assistance. Journal of Consulting and Clinical Psychology, 54(4), 416-423.

Triandis, H. C. (1995). Individualism and Collectivism. Boulder, CO: Westview. Todd, M., Armeli, S., Tennen, H., Carney, M.A., \& Affleck, G. (2003). A daily diary validity test of drinking to cope measures. Psychology of Addictive Behaviors, 17(4), 303-311.

Todd, M., Armeli, S., Tennen, H., Carney, M. A., Ball, S. A., Kranzler, H. R., \& Affleck, G. (2005). Drinking to cope: A comparison of questionnaire and electronic diary reports. Journal of Studies on Alcohol, 66, 121-129.

Turner, R.J. Frankel, B.G., \& Levin, D.M. (1983). Social support: Conceptualization, measurement, and implications for mental health. In Greenley, J.R. (Ed). Research in Community and Mental Health, (pp. 67111). Greenwich, CT: JAI Press Turner, R.J. Uchino \& Kazdin (2004) Social support and physical health. Yale University Press 
Daily Social Support and Drinking, 182

United State Department of Agriculture, (1997). Does alcohol have a place in a healthy diet. Retrieved from: http://www.cnpp.usda.gov/Publications /NutritionInsights/insight4.pdf

Vaux, A., (1988). Social Support: Theory, Research, and Intervention. A. Vaux ( Ed). New York: NY, Praeger Publishing.

Vittengl, J. \& Holt, C. (1998). A time-series diary study of mood and social interaction. Motivation and Emotion, 22(3), 255-275.

Wang, M., Lui, S., Zhan, Y., Shi, J. (2010). Daily work-family conflict and alcohol use: Testing the cross-level moderation effects of peer drinking norms and social support. Journal of Applied Psychology, 95, 377-386.

Wechsler, H., Dowdall, G. W., Davenport, A., \& Rimm, E. B. (1995). A genderspecific measure of binge drinking among college students. American Journal of Public Health, 85, 982-985.

Wechsler, H., \& Nelson, T. F. (2001). Binge drinking and the American college student: What's five drinks? Psychology of Addictive Behaviors, 15, 287291.

Wellman, B. \& Wortley, S. (1990). Different strokes from different folks: community ties and social support. American Journal of Sociology, 96(3), $558-588$.

West, S. \&Hepworth, J.T. (1991). Statistical issues in the study of temporal data: Daily experiences. Journal of Personality, 59(3), 609-662. 
Daily Social Support and Drinking, 183

West, J.A. \& Sutker, P.B. (1990). Incentive motivation, affective change, and alcohol use: A model. In M. Cox (Ed.), Why people drink (pp. 291-311), New York: Gardner Press.

Westen, D. (1994). Toward an integrative model of affect regulation: Applications to social-psychological research. Journal of Personality, 62, 641-667.

Wheaton, B. (1985). Models for the stress-buffering function of coping resources. Journal of Health and Social Behavior, 26, 352 - 364.

Wethington, E., \& Kessler, R. C. (1986). Perceived support, received support, and adjustment to stressful life events. Journal of Health and Social Behavior, 27, 78-89.

Wheeler, L., \& Reis, H.T. (1991). Self-recording of events in everyday life: Origins, types, and uses. Journal of Personality, 59(3), 339-354.

Windle, M., \& Windle, R.C. (1996). Coping strategies, drinking motives, and stressful life events among adolescents: Associations with emotional and behavioral problems, and academic functioning. Journal of Abnormal Psychology, 105, 551-560.

Wills, T. A., Resko, J., Ainette, M., \& Mendoza, D. (2004). The role of parent and peer support in adolescent substance use: A test of mediated effects. Psychology of Addictive Behaviors, 18, 122-134. 
Daily Social Support and Drinking, 184

Wills, T.A., \& Shinar, O. (2000). Measuring perceived and received support. In S. Cohen, L. Underwood, \& B. Gottlieb (Eds.), Measuring and intervening in social support, (pp. 86-135). New York: Oxford University Press.

Wilsnack, R.W. \& Cheloha, R. (1987). Women's roles and problem drinking across the life span. Social Problems, 34, 231-248

Wittenbrink, B., \& Schwarz, N. (2007). Implicit measures of attitudes: Procedures and controversies. New York: Guilford.

Wortman, C.B. \& Lehman, D.R. (1985). Reactions to victims of life crises: Support attempts that fail. In I.G Sarason \& B.R. Sarason (Eds.), Social Support: Theory, Research and Applications (pp. 463 - 489). Dordrecht, The Netherlands: Martinus Nijhoff.

Yuan, J-M., Ross, R.K. \& Gao, Y. (1997). Follow up study of moderate alcohol intake and mortality among middle aged men in Shanghai, China. British Medical Journal, 314, 18-23. 
Daily Social Support and Drinking, 185

Appendix A. Sample recruitment advertisement.

Health Study: Earn up to $\$ 185$ and a chance to win!

Call now to see if you are eligible to participate in a study about adult daily behavior! The study takes just a few minutes each day for 30 days and you can earn up to $\$ 185$, with a chance to win a $\$ 500$ prize! If you are over 21 and would like the opportunity to contribute to this study about adult health behaviors (e.g., exercise, mood, alcohol consumption), please contact Dr. Cynthia Mohr at Portland State University at 503-725-3986, or email 
Daily Social Support and Drinking, 186

Appendix B. Handheld interviewer, interpersonal exchange questions

How negative were the following interactions with people since the last interview?

1. Yelled at me

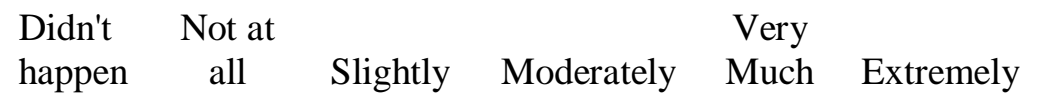

2. Nagged me

$\begin{array}{ll}0 & 1\end{array}$

3. Blocked my goals

$0 \quad 1$

4. Took my feelings lightly

5. Impatient with me

6. Hurtful to others

7. Had argument

8. Being ignored

9. Other

$0 \quad 1$

2

23

2

2

3

4

45

$4 \quad 5$

$4 \quad 5$

0

$\begin{array}{ll}0 & 1\end{array}$

0

$0 \quad 1$

$\begin{array}{ll}0 & 1 \\ 0 & 1 \\ 0 & 1\end{array}$

Didn't Not happen at all

1. Helped me

$0 \quad 1$

Slightly

Moderately
3

Very Much Extremely

2. Spent time/hung out with me

3. Shared affection/love

4. Pleasant conversation

5. Received compliment

6. Expressed interest

7. Helped others

8. Other

$\begin{array}{ll}0 & 1 \\ 0 & 1 \\ 0 & 1 \\ 0 & 1 \\ 0 & 1 \\ 0 & 1 \\ 0 & 1\end{array}$

2
2
2
2
2
2
2

3
3
3
3
3
3
3

5

5

5

$4 \quad 5$

45

$4 \quad 5$

$4 \quad 5$

45

45

45

5 